\title{
RAMIFICATION AND NEARBY CYCLES FOR $\ell$-ADIC SHEAVES ON RELATIVE CURVES
}

\author{
HAOYU HU
}

(Received July 24, 2013, revised March 4, 2014)

\begin{abstract}
Deligne and Kato proved a formula computing the dimension of the nearby cycles complex of an $\ell$-adic sheaf on a relative curve over an excellent strictly henselian trait. In this article, we reprove this formula using Abbes-Saito's ramification theory.
\end{abstract}

\section{CONTENTS}

1. Introduction 153

2. Notation 157

3. Kato's Swan conductors with differential values 157

4. Abbes-Saito's ramification theory 162

5. Ramification of extensions of type (II) 165

6. Tubular neighborhoods and normalized integral models 169

7. Isogenies associated to extensions of type (II): the equal characteristic case $\quad 170$

8. Isogenies associated to extensions of type (II): the unequal characteristic case 176

9. The refined Swan conductor of an extension of type (II) 184

10. Comparison of Kato's and Abbes-Saito's characteristic cycles 186

11. Nearby cycles of $\ell$-sheaves on relative curves $\quad 190$

$\begin{array}{ll}\text { References } & 192\end{array}$

\section{Introduction.}

1.1. Let $R$ be an excellent strictly henselian discrete valuation ring of residue characteristic $p>0, S=\operatorname{Spec}(R), s$ (resp. $\eta$, resp. $\bar{\eta}$ ) the closed point (resp. the generic point, resp. a geometric generic point) of $S$. Let $\mathfrak{X}$ be a smooth relative curve over $S, x$ a closed point of the special fiber $\mathfrak{X}_{s}, X$ the strict henselization of $\mathfrak{X}$ at $x, U$ a non-empty open sub-scheme of $X_{\eta}$, and $u: U \rightarrow X_{\eta}$ the canonical injection. Let $\Lambda$ be a finite field of characteristic $\ell \neq p$, and $\mathscr{F}$ a locally constant constructible étale sheaf of $\Lambda$-modules on $U$. The spaces of nearby cycles of $\mathscr{F}$

$$
\Psi_{x}^{i}\left(u_{!} \mathscr{F}\right)=\mathrm{H}_{\mathrm{e} t}^{i}\left(X_{\bar{\eta}}, u_{!} \mathscr{F}\right) \quad(i \geqslant 0)
$$

vanish when $i \geqslant 2$ ([SGA7II, XIII], [Fu, 9.2.2]) and the dimension of $\Psi_{x}^{0}(u ! \mathscr{F})$ is easy to compute. The aim of this article is to reprove a Deligne-Kato's formula that computes

2010 Mathematics Subject Classification. Primary 14F20; Secondary 11S15.

Key words and phrases. Nearby cycles, ramification, characteristic cycles, Deligne-Kato's formula. 
the dimension of $\Psi_{x}^{1}(u ! \mathscr{F})$ [Lau1, Kato1, Kato2] using Abbes-Saito's ramification theory [AS1, AS2].

1.2. Let $\mathfrak{p}$ be the generic point of the special fiber $X_{s}$. We denote by $\kappa(\mathfrak{p})$ the residue field of $\mathfrak{p}$, which is the fraction field of a strictly henselian discrete valuation ring. Assume first that $\mathscr{F}$ can be extended to a locally constant constructible sheaf $\widetilde{\mathscr{F}}$ on an open sub-scheme $\widetilde{U}$ of $X$ containing $\mathfrak{p}$. Then Deligne computes the dimension of $\Psi_{x}^{1}\left(u_{!} \mathscr{F}\right)$. Let $\operatorname{sw}_{x}(\widetilde{\mathscr{F}})$ be the Swan conductor of the pull-back of $\widetilde{\mathscr{F}}$ on $\operatorname{Spec}(\kappa(\mathfrak{p}))$ and let

$$
\varphi(s)=\operatorname{sw}_{x}(\widetilde{\mathscr{F}})+\operatorname{rank}(\mathscr{F}) .
$$

On the other hand, for any $t \in X_{\bar{\eta}}-U_{\bar{\eta}}$, let $\operatorname{sw}_{t}(\mathscr{F})$ be the Swan conductor of the pull-back of $\mathscr{F}$ on $\operatorname{Spec}\left(\mathcal{O}_{X_{\bar{\eta}}, t}\right) \times_{X} U$, and let

$$
\varphi(\eta)=\sum_{t \in X_{\bar{\eta}}-U_{\bar{\eta}}}\left(\operatorname{sw}_{t}(\mathscr{F})+\operatorname{rank}(\mathscr{F})\right) .
$$

Then, Deligne's formula is ([Lau1, 5.1.1])

$$
\operatorname{dim}_{\Lambda} \Psi_{x}^{0}(u ! \mathscr{F})-\operatorname{dim}_{\Lambda} \Psi_{x}^{1}(u ! \mathscr{F})=\varphi(s)-\varphi(\eta) .
$$

1.3. Kato generalized Deligne's formula for any $\mathscr{F}$. His formula has the same form as (1.2.1). The definition of the invariant $\varphi(\eta)$ is the same as above, but $\varphi(s)$ cannot be defined by the same method. He provided two definitions of $\varphi(s)$. The first one uses a ramification theory for valuation rings of rank two, which he developed for this purpose [Kato1]. The second one uses his notion of Swan conductors with differential values [Kato2]. Both methods rely on Epp's partial semi-stable reduction theorem [Epp]. In this article, we define the invariant $\varphi(s)$ in terms of ramification theory of Abbes and Saito [AS1, AS2]. The case when $\mathscr{F}$ has rank 1 is due to Abbes and Saito ([AS4, Appendix A]).

1.4. Let $K$ be a complete discrete valuation field, $\mathcal{O}_{K}$ its integer ring, $\mathfrak{m}_{K}$ the maximal ideal of $\mathcal{O}_{K}$ and $F$ the residue field of $\mathcal{O}_{K}$. We assume that $F$ is of finite type over a perfect field $F_{0}$ of characteristic $p$. We denote by $\bar{K}$ a separable closure of $K$, by $\mathcal{O}_{\bar{K}}$ the integral closure of $\mathcal{O}_{K}$ in $\bar{K}$, by $\bar{F}$ the residue field of $\mathcal{O}_{\bar{K}}$, by $v$ the valuation of $\bar{K}$ normalized by $v\left(K^{\times}\right)=\mathbb{Z}$ and by $G_{K}$ the Galois group of $\bar{K} / K$. Abbes and Saito defined a decreasing filtration $G_{K, \log }^{r}\left(r \in \mathbb{Q}_{\geqslant 0}\right)$ of $G_{K}$, called the logarithmic ramification filtration. For any rational number $r \geqslant 0$, we put $G_{K, \log }^{r+}=\overline{\bigcup_{b>r} G_{K, \log }^{b}}$. Then $P=G_{K, \log }^{0+}$ is the wild inertia subgroup of $G_{K}$ ([AS1, 3.15]). For any rational number $r>0$, the graded piece

$$
\mathrm{Gr}_{\log }^{r} G_{K}=G_{K, \log }^{r} / G_{K, \log }^{r+}
$$

is abelian and killed by $p$ ([Sa2, 1.24], [Sa3, Theorem 2]).

For any $r \in \mathbb{Q}$, we denote by $\mathfrak{m}_{\bar{K}}^{r}$ (resp. $\mathfrak{m}_{\bar{K}}^{r+}$ ) the set of elements of $\bar{K}$ such that $v(x) \geqslant r$ (resp. $v(x)>r$ ). Let $\Omega_{F}^{1}(\log )$ be the $F$-vector space

$$
\Omega_{F}^{1}(\log )=\left(\Omega_{F / F_{0}}^{1} \oplus\left(F \otimes_{\mathbb{Z}} K^{\times}\right)\right) /\left(\mathrm{d} \bar{a}-\bar{a} \otimes a ; a \in \mathcal{O}_{K}^{\times}\right),
$$


where $\bar{a}$ is the residue class of $a$ in $F$. We have a canonical exact sequence of finite dimensional $F$-vector spaces

$$
0 \rightarrow \Omega_{F}^{1} \rightarrow \Omega_{F}^{1}(\log ) \rightarrow F \rightarrow 0 .
$$

For any rational number $r>0$, there exists a canonical injective homomorphism ([Sa2, 1.24], [Sa3, Theorem 2]), called the refined Swan conductor,

$$
\text { rsw : } \operatorname{Hom}_{\mathbb{F}_{p}}\left(\mathrm{Gr}_{\log }^{r} G_{K}, \mathbb{F}_{p}\right) \rightarrow \Omega_{F}^{1}(\log ) \otimes_{F} \mathfrak{m}_{\bar{K}}^{-r} / \mathfrak{m}_{\bar{K}}^{-r+} .
$$

Let $M$ be a finite dimensional $\Lambda$-vector space on which $P$ acts through a finite discrete quotient,

$$
M=\oplus_{r \in \mathbb{Q}_{\geqslant 0}} M^{(r)}
$$

the slope decomposition of $M$ (cf. Lemma 4.5), and for any rational number $r>0$,

$$
M^{(r)}=\oplus_{\chi} M_{\chi}^{(r)}
$$

the central character decomposition of $M^{(r)}$, where the sum runs over finitely many characters $\chi: \mathrm{Gr}_{\log }^{r} G_{K} \rightarrow \Lambda_{\chi}^{\times}$such that $\Lambda_{\chi}$ is a finite extension of $\Lambda$ (cf. Lemma 4.7). Enlarging $\Lambda$, we may assume that for all rational number $r>0$ and for all central characters $\chi$ of $M^{(r)}$, $\Lambda=\Lambda_{\chi}$. We fix a non-trivial character $\psi_{0}: \mathbb{F}_{p} \rightarrow \Lambda^{\times}$. Since $\operatorname{Gr}_{\log }^{r} G_{K}$ is abelian and killed by $p, \chi$ factors uniquely through $\mathrm{Gr}_{\log }^{r} G_{K} \rightarrow \mathbb{F}_{p} \stackrel{\psi_{0}}{\rightarrow} \Lambda^{\times}$. We denote abusively by $\chi: \mathrm{Gr}_{\log }^{r} G_{K} \rightarrow \mathbb{F}_{p}$ the induced character. We fix a uniformizer $\pi$ of $\mathcal{O}_{K}$. We define AbbesSaito's characteristic cycle of $M$ and denote by $\mathrm{CC}_{\psi_{0}}(M)$ the following section (4.12.1)

$$
\mathrm{CC}_{\psi_{0}}(M)=\bigotimes_{r \in \mathbb{Q}_{>0}} \bigotimes_{\chi}\left(\operatorname{rsw}(\chi) \otimes \pi^{r}\right)^{\operatorname{dim}_{\Lambda} M_{\chi}^{(r)}} \in\left(\Omega_{F}^{1}(\log ) \otimes_{F} \bar{F}\right)^{\otimes \operatorname{dim}_{A} M / M^{(0)}} .
$$

1.5. In the following, we assume that $p$ is not a uniformizer of $K$ (i.e., either $K$ has characteristic $p$ or $K$ has characteristic zero and $p$ is not a uniformizer of $\mathcal{O}_{K}$ ). Let $L$ be a finite Galois extension of $K$ of group $G$. We assume that $L / K$ has ramification index one and that the residue field extension is non-trivial, purely inseparable and monogenic; we say that the extension $L / K$ is of type (II) (cf. Subsection 3.3). Let $M$ be a finite $\Lambda$-vector space on which $G_{K}$ acts through $G$. We prove that, for any rational number $r>0$, and any central character $\chi: \mathrm{Gr}_{\log }^{r} G_{K} \rightarrow \mathbb{F}_{p}$ of $M^{(r)}$, we have (Proposition 5.7)

$$
\operatorname{rsw}(\chi) \in \Omega_{F}^{1} \otimes_{F} \mathfrak{m}_{\bar{K}}^{-r} / \mathfrak{m}_{\bar{K}}^{-r+} .
$$

Hence, we have $\mathrm{CC}_{\psi_{0}}(M) \in\left(\Omega_{F}^{1} \otimes_{F} \bar{F}\right)^{\otimes m}$, where $m=\operatorname{dim}_{A} M / M^{(0)}$ (Corollary 5.10). On the other hand, using Kato's theory of Swan conductors with differential values, we can define Kato's characteristic cycle $\mathrm{KCC}_{\psi_{0}(1)}(M)$ (3.17.1). Our main result (10.7.4) is the following equality

$$
\mathrm{CC}_{\psi_{0}}(M)=\mathrm{KCC}_{\psi_{0}(1)}(M) .
$$

Using Kato's theory, we deduce a Hasse-Arf type theorem (Corollary 10.5)

$$
\mathrm{CC}_{\psi_{0}}(M) \in\left(\Omega_{F}^{1}\right)^{m} \subset\left(\Omega_{F}^{1} \otimes_{F} \bar{F}\right)^{m},
$$

and an induction formula (10.6.1) for Abbes-Saito's characteristic cycle. 
1.6. Under the assumptions of Subsection 1.1, we can now give a new definition of $\varphi(s)$. Firstly, by Epp's results [Epp], we can reduce to the case where $\mathscr{F}$ is trivialized by a Galois étale connected covering $U^{\prime}$ of $U$ such that the special fiber of the normalization $X^{\prime}$ of $X$ in $U^{\prime}$ is reduced. We denote by $\widehat{\mathcal{O}}_{X, \mathfrak{p}}$ the completion of $\mathcal{O}_{X, \mathfrak{p}}$, by $K_{\mathfrak{p}}$ the fraction field of $\widehat{\mathcal{O}}_{X, \mathfrak{p}}$ and by $\mathscr{F}_{\mathfrak{p}}$ the representation of $\operatorname{Gal}\left(K_{\mathfrak{p}}^{\text {sep }} / K_{\mathfrak{p}}\right)$ corresponding to the pull-back of $\mathscr{F}$ on $\operatorname{Spec}\left(\widehat{\mathcal{O}}_{X, \mathfrak{p}}\right) \times_{X} U$. The latter factors through the Galois group of a finite Galois extension $L_{\mathfrak{p}}$ of $K_{\mathfrak{p}}$, which is of type (II) over an unramified extension of $K_{\mathfrak{p}}$. We fix a uniformizer $\pi$ of $R$ and a non-trivial character $\psi_{0}: \mathbb{F}_{p} \rightarrow \Lambda^{\times}$. We still have $\operatorname{CC}_{\psi_{0}}\left(\mathscr{F}_{\mathfrak{p}}\right) \in\left(\Omega_{\kappa(\mathfrak{p})}^{1}\right)^{\otimes m}$ (cf. Remark 10.7). We denote by $\operatorname{ord}_{\mathfrak{p}}$ the valuation of $\kappa(\mathfrak{p})$ normalized by $\operatorname{ord}_{\mathfrak{p}}\left(\kappa(\mathfrak{p})^{\times}\right)=\mathbb{Z}$ and abusively by $\operatorname{ord}_{\mathfrak{p}}: \Omega_{\kappa(\mathfrak{p})}^{1}-\{0\} \rightarrow \mathbb{Z}$ the map defined by $\operatorname{ord}_{\mathfrak{p}}(\alpha \mathrm{d} \beta)=\operatorname{ord}_{\mathfrak{p}}(\alpha)$, if $\alpha, \beta \in \kappa(\mathfrak{p})^{\times}$and $\operatorname{ord}_{\mathfrak{p}}(\beta)=1$. The latter can be uniquely extended to $\left(\Omega_{\kappa(\mathfrak{p})}^{1}\right)^{\otimes r}-\{0\}$ for any integer $r \geqslant 1$. We denote by $\overline{\mathscr{F}}_{\mathfrak{p}}$ the restriction to $\operatorname{Spec}(\kappa(\mathfrak{p}))$ of the direct image of $\mathscr{F}_{\mathfrak{p}}$ by the map $\operatorname{Spec}\left(K_{\mathfrak{p}}\right) \rightarrow \operatorname{Spec}\left(\widehat{\mathcal{O}}_{X, \mathfrak{p}}\right)$. It corresponds to a representation of $\operatorname{Gal}(\overline{\kappa(\mathfrak{p})} / \kappa(\mathfrak{p}))$. The invariant $\varphi(s)$ is defined by

$$
\varphi(s)=-\operatorname{ord}_{\mathfrak{p}}\left(\mathrm{CC}_{\psi_{0}}\left(\mathscr{F}_{\mathfrak{p}}\right)\right)+\operatorname{sw}_{x}\left(\overline{\mathscr{F}}_{\mathfrak{p}}\right)+\operatorname{rank}\left(\overline{\mathscr{F}}_{\mathfrak{p}}\right) .
$$

In fact, Kato's second definition of $\varphi(s)([\mathrm{Kato} 2,4.4])$ is obtained by replacing $\mathrm{CC}_{\psi_{0}}\left(\mathscr{F}_{\mathfrak{p}}\right)$ by $\operatorname{KCC}_{\psi_{0}(1)}\left(\mathscr{F}_{\mathfrak{p}}\right)$ in (1.6.1). Hence, from (1.5.1), we deduce that Deligne-Kato's formula (1.2.1) holds true with our definition (cf. Theorem 11.9).

1.7. Deligne-Kato's formula has already had important applications. For instance, Deligne's formula could be used in Laumon's work on local Fourier transform ([Lau2, 2.4.3]) and Kato's formula was recently used in the work of Obus and Wewers on local lifting problem [OW]. We would like to mention that Laumon's formula of the rank of the local Fourier transform is a direct application of the formulation of Deligne-Kato's formula using (1.6.1). Indeed, it was reproved in ([AS4, Appendix B]) by reducing to the rank 1 case by Brauer theorem.

1.8. This article is organized as follows. We briefly introduce Kato's Swan conductors with differential values and Abbes-Saito's ramification theory in $\S 3$ and $\S 4$, respectively. We study in $\$ 5$ the ramification of extensions of type (II). We recall tubular neighborhoods and normalized integral models in $\S 6$. We study the isogeny associated to an extension of type (II) in $\$ 7$ in the equal character case and in $\$ 8$ in the unequal characteristic case. Using the results of these two sections, we prove the main theorem 5.9 in $\S 9$. In $\$ 10$, the heart of this article, we compare Kato's characteristic cycle and Abbes-Saito's characteristic cycle. The last section is devoted to Deligne-Kato's formula by using Abbes-Saito's characteristic cycle.

Acknowledgment. This article is a part of the author's thesis at Universite Paris-Sud and Nankai University. The author would like to express his deepest gratitude to his supervisors Ahmed Abbes and Lei Fu for leading him to this area and for patiently guiding him in solving this problem. The author is also grateful to Fonds Chern and Fondation Mathématiques Jacques Hadamard for their support during his stay in France. 


\section{Notation.}

2.1. In this article, $K$ denotes a complete discrete valuation field, $\mathcal{O}_{K}$ its integer ring, $\mathfrak{m}_{K}$ the maximal ideal of $\mathcal{O}_{K}$ and $F$ the residue field of $\mathcal{O}_{K}$. We assume that the characteristic of $F$ is $p>0$. We fix a uniformizer $\pi$ of $\mathcal{O}_{K}$. Let $\bar{K}$ be a separable closure of $K, G_{K}$ the Galois group of $\bar{K}$ over $K, \mathcal{O}_{\bar{K}}$ the integral closure of $\mathcal{O}_{K}$ in $\bar{K}, \bar{F}$ the residue field of $\mathcal{O}_{\bar{K}}$ and $v$ the valuation of $\bar{K}$ normalized by $v\left(K^{\times}\right)=\mathbb{Z}$. We denote by $\mathrm{FE}_{/ K}$ the category of finite étale $K$-algebras. For any object $K^{\prime}$ of $\mathrm{FE}_{/ K}$, we denote by $\mathcal{O}_{K^{\prime}}$ the integer ring of $K^{\prime}$ and by $\mathfrak{m}_{K^{\prime}}$ the radical of $\mathcal{O}_{K^{\prime}}$.

2.2. For a field $k$ and one dimensional $k$-vector spaces $V_{1}, \ldots, V_{m}$, we denote by $k\left\langle V_{1}, \ldots, V_{m}\right\rangle$ the $k$-algebra

$$
\bigoplus_{\left(i_{1}, \ldots, i_{m}\right) \in \mathbb{Z}^{m}} V_{1}^{\otimes i_{1}} \otimes \cdots \otimes V_{m}^{\otimes i_{m}},
$$

and by $\left(k\left\langle V_{1}, \ldots, V_{m}\right\rangle\right)^{\times}$its group of units. An element of $\left(k\left\langle V_{1}, \ldots, V_{m}\right\rangle\right)^{\times}$is contained in some vector space $V_{1}^{\otimes i_{1}} \otimes \cdots \otimes V_{m}^{\otimes i_{m}}$. Such an element $x$ will be denoted by $[x]$ and we adopt the additive notation, i.e. $[x]+[y]=[x \cdot y]$ and $-[x]=\left[x^{-1}\right]$. If for each $1 \leqslant i \leqslant m, e_{i}$ is a non-zero element of $V_{i}$, we have an isomorphism

$$
k\left\langle V_{1}, \ldots, V_{m}\right\rangle \stackrel{\sim}{\rightarrow} k\left[X_{1}, \ldots, X_{m}, X_{1}^{-1}, \ldots, X_{m}^{-1}\right], \quad e_{i} \mapsto X_{i},
$$

and hence an isomorphism

$$
\left(k\left\langle V_{1}, \ldots, V_{m}\right\rangle\right)^{\times} \stackrel{\sim}{\rightarrow} k^{\times} \oplus \mathbb{Z}^{m} .
$$

\section{Kato's Swan conductors with differential values.}

3.1. In this section, we fix a finite separable extension $L$ of $K$ of ramification index $e$ contained in $\bar{K}$. We denote by $\mathcal{O}_{L}$ its integer ring and by $E$ the residue field of $\mathcal{O}_{L}$.

3.2. We denote the group $\left(F\left\langle\mathfrak{m}_{K} / \mathfrak{m}_{K}^{2}\right\rangle\right)^{\times}$by $R_{K}$ and the group $\left(E\left\langle\mathfrak{m}_{L} / \mathfrak{m}_{L}^{2}\right\rangle\right)^{\times}$by $R_{L}$ (cf. Subsection 2.2). The canonical isomorphisms

$$
\begin{gathered}
E \otimes_{F}\left(\mathfrak{m}_{K} / \mathfrak{m}_{K}^{2}\right) \stackrel{\sim}{\rightarrow} \mathfrak{m}_{L}^{e} / \mathfrak{m}_{L}^{e+1}, \\
\left(\mathfrak{m}_{L} / \mathfrak{m}_{L}^{2}\right)^{\otimes e} \stackrel{\sim}{\rightarrow} \mathfrak{m}_{L}^{e} / \mathfrak{m}_{L}^{e+1},
\end{gathered}
$$

induce an injective homomorphism of $F$-algebras

$$
F\left\langle\mathfrak{m}_{K} / \mathfrak{m}_{K}^{2}\right\rangle \rightarrow E\left\langle\mathfrak{m}_{L} / \mathfrak{m}_{L}^{2}\right\rangle
$$

and hence an injective homomorphism $R_{K} \rightarrow R_{L}$.

3.3. Kato's theory applies if the extension $L / K$ is of one of the following types ([Kato2, $1.5]):$

(I) $L / K$ is totally ramified (i.e., $F=E$ );

(II) the ramification index of $L / K$ is 1 and the residue field extension $E / F$ is purely inseparable and monogenic. 
Observe that in both cases, $\mathcal{O}_{L}$ is monogenic over $\mathcal{O}_{K}$. These two cases do not cover all finite separable extensions.

In the remaining part of this section, we assume that $L / K$ is of type (II). We denote by $p^{n}$ the degree of the residue extension $E / F$. We choose an element $h \in \mathcal{O}_{L}$ such that its reduction $\bar{h} \in E$ is the generator of $E / F$ and a lifting $a \in \mathcal{O}_{K}$ of $\bar{a}=\bar{h}^{p^{n}} \in F$.

LEMMA 3.4. Let $V$ be the kernel of the canonical morphism $\Omega_{F}^{1} \rightarrow \Omega_{E}^{1}$. Denote by $\varrho$ the morphism $E \rightarrow F, b \mapsto b^{p^{n}}$, by $\phi$ the morphism $F \rightarrow F, b \mapsto b^{p^{n}}$, and by $\varphi$ the morphism $E \rightarrow E, b \mapsto b^{p^{n}}$.

(i) The $F$-vector space $V$ is of dimension 1 , generated by $\mathrm{d} \bar{a}$.

(ii) The E-vector space $\Omega_{E / F}^{1}$ is of dimension 1 , generated by $\mathrm{d} \bar{h}$.

(iii) The canonical morphism $F \otimes_{\varrho, E} \Omega_{E / F}^{1} \rightarrow \Omega_{F / \phi(F)}^{1}=\Omega_{F}^{1}$ associated to $F \rightarrow E \stackrel{\varrho}{\rightarrow}$ $F$ is injective with image $V$.

(iv) For any 1-dimensional $E$ vector space $W$, the morphism

$$
E \otimes_{\varphi, E} W \rightarrow W^{\otimes p^{n}}, \quad y \otimes z \mapsto y z^{\otimes p^{n}}
$$

is an isomorphism.

(v) There exists a canonical E-linear isomorphism

$$
E \otimes_{F} V \stackrel{\sim}{\rightarrow}\left(\Omega_{E / F}^{1}\right)^{\otimes p^{n}},
$$

that maps $y \otimes \mathrm{d} \bar{a}$ to $y(\mathrm{~d} \bar{h})^{\otimes p^{n}}$.

Proof. (i), (ii), (iv) are obvious. We have two canonical exact sequences of differential modules corresponding to the extensions $\phi: F \rightarrow E \stackrel{\varrho}{\rightarrow} F$ and $\varphi: E \stackrel{\varrho}{\rightarrow} F \rightarrow E$,

$$
\begin{gathered}
F \otimes_{\varrho, E} \Omega_{E / F}^{1} \stackrel{\beta}{\rightarrow} \Omega_{F}^{1} \rightarrow \Omega_{F / \varrho(E)}^{1} \rightarrow 0, \\
E \otimes_{F} \Omega_{F / \varrho(E)}^{1} \rightarrow \Omega_{E}^{1} \rightarrow \Omega_{E / F}^{1} \rightarrow 0 .
\end{gathered}
$$

Since the canonical morphism $\Omega_{F}^{1} \rightarrow \Omega_{E}^{1}$ factors as

$$
\Omega_{F}^{1} \rightarrow \Omega_{F / \varrho(E)}^{1} \rightarrow E \otimes_{F} \Omega_{F / \varrho(E)}^{1} \rightarrow \Omega_{E}^{1},
$$

the image of $F \otimes_{\varrho, E} \Omega_{E / F}^{1}$ in $\Omega_{E}^{1}$ is $\{0\}$. Hence the image of $\beta$ lies in $V$. Since the kernel of $\Omega_{F}^{1} \rightarrow \Omega_{F / \varrho(E)}^{1}$ is not zero (as it contains $\mathrm{d} \bar{a}$ ) and since $F \otimes_{\varrho, E} \Omega_{E / F}^{1}$ is of dimension $1, \beta$ is injective. Hence $\beta$ induces an isomorphism

$$
\beta: F \otimes_{\varrho, E} \Omega_{E / F}^{1} \stackrel{\sim}{\rightarrow} V .
$$

From (ii) and (iv), we obtain an isomorphism

$$
\beta^{\prime}: E \otimes_{\varphi, E} \Omega_{E / F}^{1} \rightarrow\left(\Omega_{E / F}^{1}\right)^{\otimes p^{n}}, \quad y \otimes z \mathrm{~d} \bar{h} \mapsto y z^{p^{n}}(\mathrm{~d} \bar{h})^{\otimes p^{n}} .
$$

We take for (3.4.1) the isomorphism $\beta^{\prime} \circ\left(\operatorname{id}_{E} \otimes \beta\right)^{-1}$. 
3.5. Let $V$ be the kernel of the canonical morphism $\Omega_{F}^{1} \rightarrow \Omega_{E}^{1}$ (Lemma 3.4). We put (Subsection 2.2)

$$
S_{K, L}=\left(F\left\langle\mathfrak{m}_{K} / \mathfrak{m}_{K}^{2}, V\right\rangle\right)^{\times} \quad \text { and } \quad S_{L / K}=\left(E\left\langle\mathfrak{m}_{L} / \mathfrak{m}_{L}^{2}, \Omega_{E / F}^{1}\right\rangle\right)^{\times} .
$$

From (3.2.1) and (3.4.1), we obtain an injective homomorphism of $F$-algebras

$$
F\left\langle\mathfrak{m}_{K} / \mathfrak{m}_{K}^{2}, V\right\rangle \hookrightarrow E\left\langle\mathfrak{m}_{L} / \mathfrak{m}_{L}^{2}, \Omega_{E / F}^{1}\right\rangle,
$$

which induces an injective homomorphism

$$
S_{K, L} \hookrightarrow S_{L / K} .
$$

3.6. Let $L^{\prime}$ be a subfield of $L$ containing $K, \mathcal{O}_{L^{\prime}}$ its integer ring and $E^{\prime}$ its residue field. When $L^{\prime} \neq L$ (resp. $L^{\prime} \neq K$ ), the extension $L / L^{\prime}$ (resp. $L^{\prime} / K$ ) is of type (II) ; we consider $S_{L^{\prime}, L}$ (resp. $S_{L^{\prime} / K}$ ) as a subgroup of $S_{L / K}$ containing $S_{K, L}$, by functoriality. If $K \neq L^{\prime} \neq L$, the following canonical maps

$$
\begin{aligned}
& \operatorname{ker}\left(\Omega_{F}^{1} \rightarrow \Omega_{E^{\prime}}^{1}\right) \rightarrow \operatorname{ker}\left(\Omega_{F}^{1} \rightarrow \Omega_{E}^{1}\right), \\
& \Omega_{E / F}^{1} \rightarrow \Omega_{E / E^{\prime}}^{1}, \\
& \operatorname{ker}\left(\Omega_{E^{\prime}}^{1} \rightarrow \Omega_{E}^{1}\right) \rightarrow \Omega_{E^{\prime} / F}^{1}
\end{aligned}
$$

are isomorphisms by considering dimensions, which give the following relations:

$$
S_{K, L}=S_{K, L^{\prime}} \subset S_{L^{\prime} / K}=S_{L^{\prime}, L} \subset S_{L / L^{\prime}}=S_{L / K} .
$$

3.7. Let $i$ be the maximal integer such that $\operatorname{Tr}_{L / K}\left(m_{L}^{i}\right)=O_{K}$. The surjective homomorphism $\operatorname{Tr}_{L / K}: \mathfrak{m}_{L}^{i} / \mathfrak{m}_{L}^{i+1} \rightarrow O_{K} / \mathfrak{m}_{K}=F$ induces an $E$-isomorphism

$$
\mathfrak{m}_{L}^{i} / \mathfrak{m}_{L}^{i+1} \stackrel{\sim}{\rightarrow} \operatorname{Hom}_{F}(E, F), \quad b \mapsto\left(a \mapsto \operatorname{Tr}_{L / K}(a b)\right),
$$

and hence a basis of $\left(\mathfrak{m}_{L} / \mathfrak{m}_{L}^{2}\right)^{\otimes(-i)} \otimes_{E} \operatorname{Hom}_{F}(E, F)$, that we call Kato's different of $L / K$ and denote by $\mathfrak{D}(L / K)$ ([Kato2, 2.1]).

3.8. Following Kato ([Kato2, 2.3]), there is an $F$-linear map $\operatorname{Tr}_{E / F}: \Omega_{E}^{1} \rightarrow \Omega_{F}^{1}$ characterized by

$$
\operatorname{Tr}_{E / F}\left(\frac{\mathrm{d} x}{x}\right)=\frac{\mathrm{d} x^{p^{n}}}{x^{p^{n}}}, \quad \operatorname{Tr}_{E / F}\left(x^{i} \frac{\mathrm{d} x}{x}\right)=0,
$$

for any $x \in E^{\times}$and $1 \leqslant i \leqslant p^{n}-1$. Its image is $V$ (Lemma 3.4) and it induces an isomorphism

$$
\Omega_{E / F}^{1} \stackrel{\sim}{\rightarrow} \operatorname{Hom}_{F}(E, V), \quad \omega \mapsto\left(a \mapsto \operatorname{Tr}_{E / F}(a \omega)\right) .
$$

Hence we obtain a sequence of isomorphisms

$\operatorname{Hom}_{F}(E, F) \stackrel{(3.8 .1)}{\longrightarrow} \Omega_{E / F}^{1} \otimes_{F} V^{\otimes(-1)} \stackrel{(3.4 .1)}{\longrightarrow} \Omega_{E / F}^{1} \otimes_{E}\left(\Omega_{E / F}^{1}\right)^{\otimes\left(-p^{n}\right)}=\left(\Omega_{E / F}^{1}\right)^{\otimes\left(1-p^{n}\right)}$,

by which $E\left\langle\mathfrak{m}_{L} / \mathfrak{m}_{L}^{2}\right\rangle \otimes_{E} \operatorname{Hom}_{F}(E, F)$ is a sub- $E\left\langle\mathfrak{m}_{L} / \mathfrak{m}_{L}^{2}\right\rangle$-module of $E\left\langle\mathfrak{m}_{L} / \mathfrak{m}_{L}^{2}, \Omega_{E / F}^{1}\right\rangle$. Hence we may consider $\mathfrak{D}(L / K)$ (Subsection 3.7) as an element of $S_{L / K}$. 
Proposition 3.9 ([Kato2, 2.2]). Let $L^{\prime}$ be a subfield of $L$ containing $K$. If $L=L^{\prime}$ (resp. $\left.L^{\prime}=K\right)$, we put $\mathfrak{D}\left(L / L^{\prime}\right)=[1]$ (resp. $\left.\mathfrak{D}\left(L^{\prime} / K\right)=[1]\right)$. Then, we have

$$
\mathfrak{D}(L / K)=\mathfrak{D}\left(L / L^{\prime}\right)+\mathfrak{D}\left(L^{\prime} / K\right) \in S_{L / K} .
$$

We consider $\mathfrak{D}\left(L^{\prime} / K\right) \in S_{L^{\prime} / K} \subseteq S_{L / K}$.

3.10. In the rest of this section, we assume that the extension $L / K$ is of Galois group $G$. For any $\sigma \in G-\{1\}$, we put

$$
s_{G}(\sigma)=[\mathrm{d} \bar{h}]-[h-\sigma(h)] \in S_{L / K},
$$

where the term $[\mathrm{d} \bar{h}]$ corresponds to the element $\mathrm{d} \bar{h}$ in $\Omega_{E / F}^{1}$ and the term $[h-\sigma(\sigma)]$ corresponds abusively to the class of $h-\sigma(h) \in\left(\mathfrak{m}_{L} / \mathfrak{m}_{L}^{2}\right)^{\otimes v(h-\sigma(h))}$. The definition of $s_{G}(\sigma)$ is independent of the choice of the generator $h$ ([Kato2, 1.8]). We also put

$$
s_{G}(1)=-\sum_{\sigma \in G-\{1\}} s_{G}(\sigma) \in S_{L / K} .
$$

We have ([Kato2, (2.4)])

$$
s_{G}(1)=\mathfrak{D}(L / K) .
$$

PROPOSITION 3.11 ([Kato2, Proposition 1.9]). Let $H$ be a normal subgroup of $G$. Then for any element $\tau \in G / H-\{1\}$, we have

$$
s_{G / H}(\tau)=\sum_{\substack{\sigma \in G \\ \sigma \mapsto \tau}} s_{G}(\sigma) .
$$

3.12. In the following of this section, let $C$ be an algebraically closed field of characteristic zero, $\xi$ a primitive $p$-th root of 1 in $C$ and $\widetilde{\mathbb{Z}}$ the integral closure of $\mathbb{Z}$ in $C$. For any finite group $H$, we denote by $R_{C}(H)$ the Grothendieck group of finitely generated $C[H]$-modules. For an element $\chi \in R(H)$, let $\langle\chi, 1\rangle=\frac{1}{\sharp H} \sum_{\sigma \in H} \operatorname{tr}_{\chi}(\sigma)$.

3.13. For an element $\chi \in R_{C}(G)$, we put

$$
\begin{aligned}
s_{G}(\chi) & =\sum_{\sigma \in G} s_{G}(\sigma) \otimes \operatorname{tr}_{\chi}(\sigma) \in S_{L / K} \otimes_{\mathbb{Z}} \widetilde{\mathbb{Z}}, \\
\varepsilon(\xi) & =\sum_{r \in \mathbb{F}_{p}^{\times} \subseteq E^{\times}}[r] \otimes \xi^{r} \in S_{L / K} \otimes_{\mathbb{Z}} \widetilde{\mathbb{Z}} .
\end{aligned}
$$

Kato defined the Swan conductor with differential values of $\chi$ as

$$
\operatorname{sw}_{\xi}(\chi)=s_{G}(\chi)+(\operatorname{dim} \chi-\langle\chi, 1\rangle) \varepsilon(\xi) \in S_{L / K} \otimes \widetilde{\mathbb{Z}} .
$$

For any $r \in \mathbb{F}_{p}^{\times}$, we have $\operatorname{sw}_{\xi} r(\chi)=\operatorname{sw}_{\xi}(\chi)+(\operatorname{dim} \chi-\langle\chi, 1\rangle)[r]$.

Proposition 3.14 ([Kato2, 3.3(1)]). Let $H$ be a normal subgroup of $G, \vartheta$ an element in $R_{C}(G / H)$ and $\vartheta^{\prime}$ the image of $\vartheta$ under the canonical map $R_{C}(G / H) \rightarrow R_{C}(G)$. Then, we have

$$
s_{G}\left(\vartheta^{\prime}\right)=s_{G / H}(\vartheta) \quad \text { and } \quad \operatorname{sw}_{\xi}\left(\vartheta^{\prime}\right)=\operatorname{sw}_{\xi}(\vartheta)
$$


Proposition 3.15 ([Kato2, 3.3(2)]). Let $H$ be a subgroup of $G$. For any $\theta \in$ $R_{C}(H)$, we have

$$
\begin{gathered}
s_{G}\left(\operatorname{Ind}_{H}^{G} \theta\right)=[G: H]\left(s_{H}(\theta)+\operatorname{dim} \theta \cdot \mathfrak{D}\left(L^{H} / K\right)\right) \\
\operatorname{sw}_{\xi}\left(\operatorname{Ind}_{H}^{G} \theta\right)=[G: H]\left(\operatorname{sw}_{\xi}(\theta)+(\operatorname{dim} \theta-\langle\theta, 1\rangle) \cdot \mathfrak{D}\left(L^{H} / K\right)\right) .
\end{gathered}
$$

By (3.10.1), (3.10.2) and (3.9.1), equation (3.15.1) can be written as

$$
\operatorname{sw}_{\xi}\left(\operatorname{Ind}_{H}^{G} \theta\right)=[G: H]\left(\operatorname{sw}_{\xi}(\theta)-(\operatorname{dim} \theta-\langle\theta, 1\rangle)\left(\sum_{\sigma \in G-H}([\mathrm{~d} \bar{h}]-[h-\sigma(h)])\right)\right) .
$$

THEOREM 3.16. ([Kato2, 3.4]). For any $\chi \in R_{C}(G)$, we have

$$
\operatorname{sw}_{\xi}(\chi) \in S_{K, L} \subset S_{L / K} \otimes_{\mathbb{Z}} \widetilde{\mathbb{Z}} .
$$

This is a generalization of Hasse-Arf's theorem. It can be reduced to the case where $G$ is cyclic of rank $p^{s}$ and $\chi$ is 1-dimensional by the induction formula (3.15.1) and Brauer theorem. Then the proof relies on the higher dimensional class field theory of Kato ([Kato2, 3.6, 3.7]).

3.17. For an element $\chi \in R_{C}(G)$, the Swan conductor with differential values $\operatorname{sw}_{\xi}(\chi)$ is given by

where

$$
\operatorname{sw}_{\xi}(\chi)=-\sharp G\left(\operatorname{dim}_{C} \chi-\langle\chi, 1\rangle\right)[\mathrm{d} \bar{h}]+\Delta,
$$

$$
\Delta=\sum_{\sigma \in G-\{1\}}[h-\sigma(h)] \otimes\left(\operatorname{dim}_{C} \chi-\operatorname{tr}_{\chi}(\sigma)\right)+\left(\operatorname{dim}_{C} \chi-\langle\chi, 1\rangle\right) \varepsilon(\xi) \in R_{L} \otimes_{\mathbb{Z}} \widetilde{\mathbb{Z}} .
$$

From (3.4.1) and Theorem 3.16, we have $\sharp G[\mathrm{~d} \bar{h}]=[\mathrm{d} \bar{a}]$ and $\Delta \in R_{K}$. Hence, we get

$$
\operatorname{sw}_{\xi}(\chi)=\left[\pi^{c}\right]+\left[\Delta^{\prime}\right]-m[\mathrm{~d} \bar{a}] \in S_{K, L},
$$

where $\pi$ is the uniformizer of $\mathcal{O}_{K}$ fixed in Subsection 2.1, $c$ is an integer, $m=\operatorname{dim}_{C} \chi-\langle\chi, 1\rangle$ and $\Delta^{\prime} \in F$ such that $\left[\pi^{c} \Delta^{\prime}\right]=\Delta$. We define Kato's characteristic cycle of $\chi$ and denote by $\mathrm{KCC}_{\xi}(\chi)$ the element

$$
\operatorname{KCC}_{\xi}(\chi)=\Delta^{\prime}(\mathrm{d} \bar{a})^{m} \in\left(\Omega_{F}^{1}\right)^{\otimes m} .
$$

REMARK 3.18 ([Kato2, 3.15]). If the extension $L / K$ is not of type (II), but there exists a subfield $K^{\prime}$ of $L$ containing $K$ such that $K^{\prime} / K$ is an unramified extension and $L / K^{\prime}$ is of type (II), we define

$$
\operatorname{sw}_{\xi}(\chi)=\operatorname{sw}_{\xi}\left(\operatorname{Res}_{\operatorname{Gal}\left(L / K^{\prime}\right)}^{G} \chi\right) .
$$

Denote by $\mathcal{O}_{K^{\prime}}$ the integer ring of $K, \mathfrak{m}_{K^{\prime}}$ the maximal ideal of $\mathcal{O}_{K^{\prime}}$ and $F^{\prime}$ the residue field of $\mathcal{O}_{K^{\prime}}$. Observe that $\operatorname{sw}_{\xi}(\chi)$ is fixed by $\operatorname{Gal}\left(K^{\prime} / K\right)$ and that the $\operatorname{Gal}\left(K^{\prime} / K\right)$-invariant part of $F^{\prime}\left\langle\mathfrak{m}_{K^{\prime}} / \mathfrak{m}_{K^{\prime}}^{2}, \operatorname{ker}\left(\Omega_{F^{\prime}}^{1} \rightarrow \Omega_{E}^{1}\right)\right\rangle$ is $F\left\langle\mathfrak{m}_{K} / \mathfrak{m}_{K}^{2}, \operatorname{ker}\left(\Omega_{F}^{1} \rightarrow \Omega_{E}^{1}\right)\right\rangle$. Thus $\operatorname{sw}_{\xi}(\chi)$ is still contained in $S_{K, L}$. 
REMARK 3.19 ([Kato2, 3.16]). Let $A$ be an algebraically closed field of characteristic $\ell \notin\{0, p\}$. We denote by $A^{\prime}$ an algebraic closure of the fraction field of the ring of Witt vectors $W(A)$. Let $\chi$ be an element of $R_{A}(G)$ and let $\hat{\chi}$ be a pre-image of $\chi$ in $R_{A^{\prime}}(G)$ ([Se2, 16.1 Theorem 33]). We denote by $\hat{\xi}$ the $p$-th root of unity in $A^{\prime}$ lifting of a primitive $p$-th root of unity $\xi$ in $A$. Then we put

$$
\operatorname{sw}_{\xi}(\chi)=\operatorname{sw}_{\hat{\xi}}(\hat{\chi}) .
$$

This definition is independent of the choice of $\hat{\chi}$ because of ([Se2, 18.2 Theorem 42]) and (3.13.1).

\section{Abbes-Saito's ramification theory.}

4.1. Abbes and Saito defined two decreasing filtrations $G_{K}^{r}$ and $G_{K, \log }^{r}\left(r \in \mathbb{Q}_{>0}\right)$ of $G_{K}$ by closed normal subgroups called the ramification filtration and the logarithmic ramification filtration, respectively ([AS1, 3.1, 3.2]).

4.2. We denote by $G_{K}^{0}$ the group $G_{K}$. For any $r \in \mathbb{Q} \geqslant 0$, we put

$$
G_{K}^{r+}=\overline{\bigcup_{s \in \mathbb{Q}_{>r}} G_{K}^{s}} \text { and } \operatorname{Gr}^{r} G_{K}=G_{K}^{r} / G_{K}^{r+} .
$$

Let $L$ be a finite separable extension of $K$. For a rational number $r \geqslant 0$, we say that the ramification of $L / K$ is bounded by $r$ (resp. by $r+$ ) if $G_{K}^{r}$ (resp. $G_{K}^{r+}$ ) acts trivially on $\operatorname{Hom}_{K}(L, \bar{K})$ via its action on $\bar{K}$. We define the conductor $c$ of $L / K$ as the infimum of rational numbers $r>0$ such that the ramification of $L / K$ is bounded by $r$. Then $c$ is a rational number and $L / K$ is bounded by $c+$ ([AS1, 6.4]). If $c>0$, the ramification of $L / K$ is not bounded by $c$.

4.3. We denote by $G_{K, \log }^{0}$ the inertia subgroup of $G_{K}$. For any $r \in \mathbb{Q} \geqslant 0$, we put

$$
G_{K, \log }^{r+}=\overline{\bigcup_{s \in \mathbb{Q}>r} G_{K, \log }^{s}} \text { and } \operatorname{Gr}_{\log }^{r} G_{K}=G_{K, \log }^{r} / G_{K, \log }^{r+} .
$$

By ([AS1, 3.15]), $P=G_{K, \log }^{0+}$ is the wild inertia subgroup of $G_{K}$, i.e., the $p$-Sylow subgroup of $G_{K, \log }^{0}$. Let $L$ be a finite separable extension of $K$. For a rational number $r \geqslant 0$, we say that the logarithmic ramification of $L / K$ is bounded by $r$ (resp. by $r+$ ) if $G_{K, \log }^{r}$ (resp. $G_{K, \log }^{r+}$ ) acts trivially on $\operatorname{Hom}_{K}(L, \bar{K})$ via its action on $\bar{K}$. We define the logarithmic conductor $c$ of $L / K$ as the infimum of rational numbers $r>0$ such that the ramification of $L / K$ is bounded by $r$. Then $c$ is a rational number and $L / K$ is bounded by $c+([\operatorname{AS} 1,9.5])$. If $c>0$, the ramification of $L / K$ is not bounded by $c$.

THEOREM 4.4 ([AS2, Theorem 1]). For every rational number $r>0$, the group $\mathrm{Gr}_{\log }^{r} G_{K}$ is abelian and is contained in the center of $P / G_{K, \log }^{r}$.

LEMMA 4.5 ([Katz, 1.1]). Let $M$ be a $\mathbb{Z}\left[\frac{1}{p}\right]$-module on which $P=G_{K, \log }^{0+}$ acts through a finite discrete quotient, say by $\rho: P \rightarrow \operatorname{Aut}_{\mathbb{Z}}(M)$. Then, 
(i) The module $M$ has a unique direct sum decomposition

$$
M=\bigoplus_{r \in \mathbb{Q} \geqslant 0} M^{(r)}
$$

into $P$-stable submodules $M^{(r)}$, such that $M^{(0)}=M^{P}$ and for every $r>0$,

$$
\left(M^{(r)}\right)^{G_{K, \log }^{r}}=0 \quad \text { and } \quad\left(M^{(r)}\right)^{G_{K, \log }^{r+}}=0 .
$$

(ii) If $r>0$, then $M^{(r)}=0$ for all but the finitely many values of $r$ for which $\rho\left(G_{K, \log }^{r+}\right) \neq$ $\rho\left(G_{K, \log }^{r}\right)$.

(iii) For any $r \geqslant 0$, the functor $M \mapsto M^{(r)}$ is exact.

(iv) For $M, N$ as above, we have $\operatorname{Hom}_{P-\bmod }\left(M^{(r)}, N^{\left(r^{\prime}\right)}\right)=0$ if $r \neq r^{\prime}$.

The decomposition (4.5.1) is called the slope decomposition of $M$. The values $r \geqslant 0$ for which $M^{(r)} \neq 0$ are called the slopes of $M$. We say that $M$ is isoclinic if it has only one slope.

4.6. In the following of this section, we fix a prime number $\ell$ different from $p$, a local $\mathbb{Z}_{\ell}$-algebra $\Lambda$ which is of finite type as a $\mathbb{Z}_{\ell}$-module and a non-trivial character $\psi_{0}: \mathbb{F}_{p} \rightarrow$ $\Lambda^{\times}$.

LEMMA 4.7 ([AS5, 6.7]). Let $M$ be a $\Lambda$-module on which $P$ acts $\Lambda$-linearly through a finite discrete quotient, which is isoclinic of slope $r>0$. So the $P$ action on $M$ factors through the group $P / G_{K, \log }^{r+}$.

(i) Let $X(r)$ be the set of isomorphism classes of finite characters $\chi: \operatorname{Gr}_{\log }^{r} G_{K} \rightarrow \Lambda_{\chi}^{\times}$ such that $\Lambda_{\chi}$ is a finite étale $\Lambda$-algebra, generated by the image of $\chi$, and having $a$ connected spectrum. Then $M$ has a unique direct sum decomposition

$$
M=\bigoplus_{\chi \in X(r)} M_{\chi}
$$

Each $M_{\chi}$ is a P stable sub- $\Lambda$-module such that $\Lambda\left[G_{K, \log }^{r}\right]$ acts on $M_{\chi}$ through $\Lambda_{\chi}$.

(ii) There are finitely many characters $\chi \in X(r)$ for which $M_{\chi} \neq 0$.

(iii) For a fixed $\chi \in X(r)$, the functor $M \rightarrow M_{\chi}$ is exact.

(iv) For $M, N$ as above, we have $\operatorname{Hom}_{\Lambda}\left(M_{\chi}, N_{\chi^{\prime}}\right)=0$ if $\chi \neq \chi^{\prime}$.

The decomposition (4.7.1) is called the central character decomposition of $M$. The characters $\chi: \mathrm{Gr}_{\log }^{r} G_{K} \rightarrow \Lambda_{\chi}^{\times}$for which $M_{\chi} \neq 0$ are called the central characters of $M$ ([AS5, $6.8])$.

Let $P_{0}$ be a finite discrete quotient of $P / G_{K, \log }^{r+}$ through which $P$ acts on $M$ and let $C_{0}$ be the image of $\mathrm{Gr}_{\log }^{r} G_{K}$ in $P_{0}$. By Theorem 4.4, we know that $C_{0}$ is contained in the center of $P_{0}$. The connected components of $\operatorname{Spec}\left(\Lambda\left[C_{0}\right]\right)$ correspond to the isomorphism classes of characters $\chi: C_{0} \rightarrow \Lambda_{\chi}^{\times}$, where $\Lambda_{\chi}$ is finite étale $\Lambda$-algebra, generated by the image of $\chi$, and having a connected spectrum. If $p^{n} C=0$, and $\Lambda$ contains a primitive $p^{n}$-th root of 1 , then $\Lambda_{\chi}=\Lambda$ for every $\chi$ such that $M_{\chi} \neq 0$.

LEMMA 4.8 ([Katz, 1.4], [AS5, 6.10]). Let A be a $\Lambda$-algebra and $M$ a left A-module on which $P$ acts A-linearly through a finite discrete quotient. Then, 
(i) In the slope decomposition $M=\bigoplus_{r} M^{(r)}$, each $M^{(r)}$ is a sub-A-module of $M$. For any A-algebra $B$, the decomposition of $B \otimes_{A} M$ is given by $B \otimes_{A} M=\bigoplus_{r}\left(B \otimes_{A}\right.$ $M(r))$.

(ii) If $M$ is isoclinic, then in the central character decomposition $M=\bigoplus_{\chi} M_{\chi}$, each $M_{\chi}$ is a sub-A-module of $M$. For any A-algebra $B$, the central character decomposition of $B \otimes_{A} M$ is given by $B \otimes_{A} M=\bigoplus_{\chi}\left(B \otimes_{A} M_{\chi}\right)$.

4.9. Let $V$ be a finite dimensional $\bar{F}$-vector space and we denote by $V^{*}$ its dual space. We consider $V$ as a smooth abelian algebraic group over $\bar{F}$, i.e. $\operatorname{Spec}\left(\operatorname{Sym}\left(V^{*}\right)\right)$. Let $\pi_{1}^{\text {alg }}(V)$ be the quotient of $\pi_{1}^{\mathrm{ab}}(V)$ classifying étale isogenies. Then $\pi_{1}^{\mathrm{alg}}(V)$ is a profinite group killed by $p$ and the group $\operatorname{Hom}\left(\pi_{1}^{\mathrm{alg}}(V), \mathbb{F}_{p}\right)$ is canonically identified with the dual space $V^{*}$ by pulling-back the Lang's isogeny $\mathbb{A}^{1} \rightarrow \mathbb{A}^{1}: t \mapsto t^{p}-t$ by linear forms (cf. [Sa4, 1.19]).

4.10. For the rest of this section, we assume that $F$ is of finite type over a perfect subfield $F_{0}$. We define the $F$-vector space $\Omega_{F}^{1}(\log )$ by

$$
\Omega_{F}^{1}(\log )=\left(\Omega_{F / F_{0}}^{1} \oplus\left(F \otimes_{\mathbb{Z}} K^{\times}\right)\right) /\left(\mathrm{d} \bar{a}-\bar{a} \otimes a ; a \in \mathcal{O}_{K}^{\times}\right) .
$$

Then we have an exact sequence of finite dimensional $F$-vector spaces

$$
0 \longrightarrow \Omega_{F}^{1} \longrightarrow \Omega_{F}^{1}(\log ) \stackrel{\text { res }}{\longrightarrow} F \longrightarrow 0,
$$

where $\operatorname{res}((0, a \otimes b))=a \cdot v(b)$ for $a \in F$ and $b \in K^{\times}$. If $K$ has characteristic $p$, we put

$$
\widehat{\Omega}_{\mathcal{O}_{K} / F_{0}}^{1}=\lim _{n} \Omega_{\left(\mathcal{O}_{K} / \mathfrak{m}_{K}^{n}\right) / F_{0}}^{1}
$$

We have an exact sequence of $F$-vector spaces

$$
0 \rightarrow \mathfrak{m}_{K} / \mathfrak{m}_{K}^{2} \rightarrow \widehat{\Omega}_{\mathcal{O}_{K} / F_{0}}^{1} \otimes_{\mathcal{O}_{K}} F \rightarrow \Omega_{F}^{1} \rightarrow 0 .
$$

If $K$ has characteristic zero and $p$ is not a uniformizer of $\mathcal{O}_{K}$, we denote by $\mathcal{O}_{K_{0}}$ the ring of Witt vectors $W\left(F_{0}\right)$ regarded as a sub-algebra of $\mathcal{O}_{K}$. Then, we put

$$
\widehat{\Omega}_{\mathcal{O}_{K} / \mathcal{O}_{K_{0}}}^{1}={\underset{\lim }{n}}_{\Omega_{\left(\mathcal{O}_{K} / \mathfrak{m}_{K}^{n}\right) / \mathcal{O}_{K_{0}}}^{1}}^{1}
$$

We have an exact sequence of $F$-vector spaces

$$
0 \rightarrow \mathfrak{m}_{K} / \mathfrak{m}_{K}^{2} \rightarrow \widehat{\Omega}_{\mathcal{O}_{K} / \mathcal{O}_{K_{0}}}^{1} \otimes_{\mathcal{O}_{K}} F \rightarrow \Omega_{F}^{1} \rightarrow 0 .
$$

For any rational number $r$, we put

$$
\begin{gathered}
\mathfrak{m}_{\bar{K}}^{r}=\{x \in \bar{K} ; v(x) \geqslant r\}, \quad \mathfrak{m}_{\bar{K}}^{r+}=\{x \in \bar{K} ; v(x)>r\}, \\
\Theta_{\bar{F}, \log }^{(r)}=\operatorname{Hom}_{F}\left(\Omega_{F}^{1}(\log ), \mathfrak{m}_{\bar{K}}^{r} / \mathfrak{m}_{\bar{K}}^{(r+)}\right), \\
\Xi_{\bar{F}}^{(r)}=\operatorname{Hom}_{F}\left(\Omega_{F}^{1}, \mathfrak{m}_{\bar{K}}^{r} / \mathfrak{m}_{\bar{K}}^{(r+)}\right) .
\end{gathered}
$$

When $K$ has characteristic $p$ (resp. characteristic zero and $p$ is not a uniformizer of $\mathcal{O}_{K}$ ), for any rational number $r>0$, we denote by $\Theta_{\bar{F}}^{(r)}$ the $\bar{F}$-vector space

$$
\Theta_{\bar{F}}^{(r)}=\operatorname{Hom}_{F}\left(\widehat{\Omega}_{\mathcal{O}_{K} / F_{0}}^{1} \otimes_{\mathcal{O}_{K}} F, \mathfrak{m}_{\bar{K}}^{r} / \mathfrak{m}_{\bar{K}}^{(r+)}\right)
$$




$$
\text { (resp. } \left.\Theta_{\bar{F}}^{(r)}=\operatorname{Hom}_{F}\left(\widehat{\Omega}_{\mathcal{O}_{K} / \mathcal{O}_{K_{0}}}^{1} \otimes_{\mathcal{O}_{K}} F, \mathfrak{m}_{K}^{r} / \mathfrak{m}_{\bar{K}}^{(r+)}\right)\right) \text {. }
$$

By (4.10.1), (4.10.2) and (4.10.3), when $p$ is not a uniformizer of $K$, we have homomorphisms

$$
\Theta_{\bar{F}, \log }^{(r)} \rightarrow \Xi_{\bar{F}}^{(r)} \rightarrow \Theta_{\bar{F}}^{(r)}
$$

By ([AS2, 5.12]), we have a canonical surjection

$$
\pi_{1}^{\mathrm{ab}}\left(\Theta_{\bar{F}, \log }^{(r)}\right) \rightarrow \mathrm{Gr}_{\log }^{r} G_{K}
$$

THEOREM 4.11 ([Sa2, 1.24], [Sa3, Theorem 2]). For every rational number $r>0$, the canonical surjection (4.10.6) factors through the quotient $\pi_{1}^{\mathrm{alg}}\left(\Theta_{\bar{F}, \log }^{(r)}\right)$. In particular, the abelian group $\mathrm{Gr}_{\log }^{r} G_{K}$ is killed by $p$ and the surjection (4.10.6) induces an injective homomorphism

$$
\text { rsw : } \operatorname{Hom}\left(\mathrm{Gr}_{\log }^{r} G_{K}, \mathbb{F}_{p}\right) \rightarrow \operatorname{Hom}_{\bar{F}}\left(\mathfrak{m}_{\bar{K}}^{r} / \mathfrak{m}_{\bar{K}}^{r+}, \Omega_{F}^{1}(\log ) \otimes \bar{F}\right) .
$$

The morphism (4.11.1) is called the refined Swan conductor.

4.12. Let $M$ be a free $\Lambda$-module of finite type on which $P$ acts $\Lambda$-linearly through a finite discrete quotient. Let

$$
M=\bigoplus_{r \in \mathbb{Q} \geqslant 0} M^{(r)}
$$

be the slope decomposition of $M$ and for each rational number $r>0$, let

$$
M^{(r)}=\bigoplus_{\chi \in X(r)} M_{\chi}^{(r)}
$$

be the central character decomposition of $M^{(r)}$. We notice that each $M_{\chi}^{(r)}$ is a free $\Lambda$-module. Enlarging $\Lambda$, we may assume that for all rational number $r>0$ and $\chi \in X(r), \Lambda=\Lambda_{\chi}$ (Lemma 4.7). Each $\chi$ factors uniquely through $\psi_{0}$ (Subsection 4.6)

$$
\mathrm{Gr}_{\log }^{r} G_{K} \rightarrow \mathbb{F}_{p} \stackrel{\psi_{0}}{\rightarrow} \Lambda^{\times}
$$

We denote abusively by $\chi$ the induced character $\operatorname{Gr}_{\log }^{r} G_{K} \rightarrow \mathbb{F}_{p}$. We define the Abbes-Saito characteristic cycle $\mathrm{CC}_{\psi_{0}}(M)$ of $M$ by

$$
\text { (4.12.1) } \mathrm{CC}_{\psi_{0}}(M)=\bigotimes_{r \in \mathbb{Q}_{>0}} \bigotimes_{\chi \in X(r)}\left(\operatorname{rsw}(\chi) \otimes \pi^{r}\right)^{\operatorname{dim}_{\Lambda} M_{\chi}^{(r)}} \in\left(\Omega_{F}^{1}(\log ) \otimes_{F} \bar{F}\right)^{\otimes \operatorname{dim}_{\Lambda} M / M^{(0)}} \text {. }
$$

\section{Ramification of extensions of type (II).}

5.1. In this section, we assume that the residue field $F$ of $\mathcal{O}_{K}$ is of finite type over a perfect field $F_{0}$ of characteristic $p$. Let $L$ be a finite Galois extension of $K$ of group $G$ and type (II) (Subsection 3.3), $\mathcal{O}_{L}$ the integer ring of $L$ and $E$ the residue field of $\mathcal{O}_{L}$. We denote by $p^{n}$ the degree of the residue extension $E / F$. We choose an element $h \in \mathcal{O}_{L}$ such that its residue class $\bar{h} \in E$ is a generator of $E / F$. We have $\mathcal{O}_{L}=\mathcal{O}_{K}[h]$. Let $f(T) \in \mathcal{O}_{K}[T]$ be the minimal polynomial of $h$ :

$$
f(T)=T^{p^{n}}+a_{p^{n}-1} T^{p^{n}-1}+\cdots+a_{0} .
$$


Notice that $\bar{a}_{0}=-\bar{h}^{p^{n}} \in F$. We put

$$
c=\sup _{\sigma \in G-\{1\}} v(h-\sigma(h))+\sum_{\sigma \in G-\{1\}} v(h-\sigma(h)),
$$

which is an integer $\geqslant p^{n}$.

For any rational number $r \geqslant 0$, we denote by $G^{r}$ (resp. $G_{\log }^{r}$ ) the image of $G_{K}^{r}$ (resp. $G_{K, \log }^{r}$ ) in $G$ ([AS1, 3.1]). Using the monogenic presentation $\mathcal{O}_{L}=\mathcal{O}_{K}[T] /(f(T))$, we obtain that, for any rational number $r>1, G^{r}=G_{\log }^{r}([\mathrm{AS} 1,3.1,3.2])$ and that the conductor of $L / K$ is $c$ ([AS1,6.6]). By Theorem 4.11, the normal subgroup $G^{c}$ of $G$ is commutative and killed by $p$. In the following, we put $\sharp G^{c}=p^{s}$.

5.2. For any integer $j \geqslant 1$, we denote by $D^{j}$ the $j$-dimensional closed poly-disc of radius one over $K$ and by $\stackrel{\circ}{D}^{j}$ the $j$-dimensional open disc of radius one over $K$. For a rational number $r \geqslant 0$, the $j$-dimensional closed poly-disc of radius $r$ is denoted by $D^{j,(r)}=$ $\left\{\left(x_{1}, \ldots, x_{j}\right) \in D^{j} ; v\left(x_{i}\right) \geqslant r\right\}$. Let

$$
\tilde{f}: D^{1} \rightarrow D^{1}, \quad x \mapsto f(x),
$$

be the morphism induced by $f$. For any rational number $r \geqslant 0$, it is easy to see that $\tilde{f}^{-1}\left(D^{1,(r)}\right)$ is a disjoint union of closed discs with the same radius, i.e. there exists a rational number $\rho(r) \geqslant 0$ such that

$$
\tilde{f}^{-1}\left(D^{1,(r)}\right)=\coprod_{1 \leqslant j \leqslant i}\left(x_{j}+D^{1,(\rho(r))}\right),
$$

where the $x_{j}$ 's are zeros of $f$. The function $\rho: \mathbb{Q} \geqslant 0 \rightarrow \mathbb{Q} \geqslant 0$ is called the Herbrand function of the extension $L / K$. By ([AS2, 6.6]), we have $\rho(c)=\sup _{\sigma \in G-\{1\}} v(h-\sigma(h))$ and

$$
G^{c}=\{\sigma \in G ; v(h-\sigma(h)) \geqslant \rho(c)\} .
$$

5.3. We denote by $u$ the map

$$
u: G \rightarrow E, \quad \sigma \mapsto \begin{cases}u_{\sigma}=\overline{\left(\frac{h-\sigma(h)}{\pi^{v(h-\sigma(h))}}\right)}, & \text { if } \sigma \neq 1, \\ u_{\sigma}=0, & \text { if } \sigma=1 .\end{cases}
$$

The restriction $\left.u\right|_{G^{c}}: G^{c} \rightarrow E$ of $u$ to $G^{c}$ is an injective homomorphism of groups. Indeed, for any $\sigma \in G^{c}-\{1\}$, we have $v(h-\sigma(h))=\rho(c)$. Hence, for $\sigma_{1}, \sigma_{2} \in G^{c}$, we have

$$
u_{\sigma_{1} \sigma_{2}}=\overline{\left(\frac{h-\sigma_{1} \sigma_{2}(h)}{\pi^{\rho(c)}}\right)}=\overline{\left(\frac{h-\sigma_{1}(h)+\sigma_{1}\left(h-\sigma_{2}(h)\right)}{\pi^{\rho(c)}}\right)}=u_{\sigma_{1}}+u_{\sigma_{2}} .
$$

Proposition 5.4. The polynomial $f_{c}(T)=f\left(\pi^{\rho(c)} T+h\right) / \pi^{c} \in L[T]$ has integral coefficients. Its reduction $\overline{f_{c}} \in E[T]$ is an additive polynomial of degree $p^{s}=\sharp G^{c}$ with a non-zero linear term.

Proof. We have

$$
f_{c}(T)=T \prod_{\sigma \in G-\{1\}} \frac{\pi^{\rho(c)} T+h-\sigma(h)}{\pi^{v(h-\sigma(h))}} \in \mathcal{O}_{L}[T] .
$$


Hence

$$
\overline{f_{c}}(T)=T \prod_{\sigma \in G-\{1\}} \overline{\left(\frac{\pi^{\rho(c)} T+h-\sigma(h)}{\pi^{v(h-\sigma(h))}}\right)}=\prod_{\sigma \in G-G^{c}} u_{\sigma} \cdot \prod_{\sigma \in G^{c}}\left(T+u_{\sigma}\right) .
$$

Choose an $\mathbb{F}_{p}$-basis $\tau_{1}, \ldots, \tau_{s}$ of $G^{c}$, we get

$$
\prod_{\sigma \in G^{c}}\left(T+u_{\sigma}\right)=\prod_{\left(j_{1}, \ldots, j_{s}\right) \in \mathbb{F}_{p}^{s}}\left(T+j_{1} u_{\tau_{1}}+\cdots+j_{s} u_{\tau_{s}}\right) .
$$

We conclude by the lemma below.

LEMMA 5.5. Let $C$ be a field of characteristic $p$. For any integer $r \geqslant 0$, let $x_{1}, \ldots, x_{r}$ be $r$ elements of $C$ such that for any $\left(j_{1}, \ldots, j_{r}\right) \in \mathbb{F}_{p}^{r}-\{0\}, j_{1} x_{1}+\cdots+j_{r} x_{r} \neq 0$. Then we have

$$
\prod_{\left(j_{1}, \ldots, j_{r}\right) \in \mathbb{F}_{p}^{r}}\left(T+j_{1} x_{1}+\cdots+j_{r} x_{r}\right)=T^{p^{r}}+\lambda_{r-1} T^{p^{r-1}}+\cdots+\lambda_{1} T^{p}+\lambda_{0} T \in C[T],
$$

where

$$
\lambda_{0}=\prod_{\left(j_{1}, \ldots, j_{r}\right) \in \mathbb{F}_{p}^{r}-\{0\}}\left(j_{1} x_{1}+\cdots+j_{r} x_{r}\right) \neq 0 .
$$

PROOF. We proceed by induction on $r$. If $r=1$,

$$
\prod_{j_{1} \in \mathbb{F}_{p}^{r}}\left(T+j_{1} x_{1}\right)=T^{p}-x_{1}^{p-1} T,
$$

which satisfies (5.5.1). Assume that (5.5.1) holds for $(r-1)$-tuples where $r \geqslant 2$, let $\left(x_{1}, \ldots, x_{r}\right) \in C^{r}$ be as in the lemma. We put

$$
g_{r-1}(T)=\prod_{\left(j_{1}, \ldots, j_{r-1}\right) \in \mathbb{F}_{p}^{r-1}}\left(T+j_{1} x_{1}+\cdots+j_{r-1} x_{r-1}\right) .
$$

Then, we have

$$
\begin{aligned}
\prod_{\left(j_{1}, \ldots, j_{r}\right) \in \mathbb{F}_{p}^{r}}\left(T+j_{1} x_{1}+\cdots+j_{r} x_{r}\right) & =\prod_{j_{r} \in \mathbb{F}_{p}}\left(g_{r-1}\left(T+j_{r} x_{r}\right)\right) \\
& =\prod_{j_{r} \in \mathbb{F}_{p}}\left(g_{r-1}(T)+j_{r} g_{r-1}\left(x_{r}\right)\right) \\
& =g_{r-1}^{p}(T)-g_{r-1}^{p-1}\left(x_{r}\right) g_{r-1}(T),
\end{aligned}
$$

which satisfies (5.5.1) since $g_{r-1}$ does.

In the following of this section, we assume that $p$ is not a uniformizer of $K$.

LEMMA 5.6. Suppose $c>2$. Then, for any $1 \leqslant i \leqslant p^{n}-1$, we have $v\left(a_{i}\right) \geqslant 2(5.1 .1)$. 
Proof. From the equation $f(T)=\prod_{\sigma \in G}(T-\sigma(h))$, for any $1 \leqslant i \leqslant p^{n}-1$, we obtain

$$
\begin{aligned}
a_{i} & =(-1)^{\left(p^{n}-i\right)} \sum_{\left\{\sigma_{1}, \ldots, \sigma_{p^{n}-i}\right\} \subseteq G} \sigma_{1}(h) \sigma_{2}(h) \cdots \sigma_{p^{n}-i}(h) \\
& =(-1)^{\left(p^{n}-i\right)} \sum_{\left\{\sigma_{1}, \ldots, \sigma_{p^{n}-i}\right\} \subseteq G}\left(\sigma_{1}(h)-h+h\right) \cdots\left(\sigma_{p^{n}-i}(h)-h+h\right) \\
& =(-1)^{\left(p^{n}-i\right)}\left(\left(\begin{array}{c}
p^{n} \\
i
\end{array}\right) h^{p^{n}-i}+\left(\begin{array}{c}
p^{n}-1 \\
i
\end{array}\right) h^{p^{n}-i-1} \sum_{\sigma \in G}(\sigma(h)-h)+\Delta\right),
\end{aligned}
$$

where $v(\Delta) \geqslant 2$. Since the integer $\left(\begin{array}{c}p^{n} \\ i\end{array}\right)$ is divisible by $p, v\left(\left(\begin{array}{c}p^{n} \\ i\end{array}\right) h^{p^{n}}\right) \geqslant 2$. Hence it is sufficient to show that

$$
v\left(\sum_{\sigma \in G}(\sigma(h)-h)\right) \geqslant 2 .
$$

Assume first that for any $\sigma \in G-\{1\}, v(h-\sigma(h))=\rho(c)$, i.e. $G=G^{c}$. It suffices to treat the case where $\rho(c)=1$. In this case, $\sharp G=c>2$ (5.1.2). From Subsection 5.1, $G$ is an $\mathbb{F}_{p}$-vector space of dimension $n$ and we choose an $\mathbb{F}_{p}$-basis $\tau_{1}, \ldots, \tau_{n}$ of $G$. By Subsection 5.3, we have

$$
\begin{aligned}
\sum_{\sigma \in G} u_{\sigma} & =\sum_{\left(j_{1}, \ldots, j_{n}\right) \in \mathbb{F}_{p}^{n}}\left(j_{1} u_{\tau_{1}}+\cdots+j_{n} u_{\tau_{n}}\right) \\
& =\frac{p^{n}(p-1)}{2}\left(u_{\tau_{1}}+\cdots+u_{\tau_{n}}\right)=0,
\end{aligned}
$$

which means that $v\left(\sum_{\sigma \in G-\{1\}}(\sigma(h)-h)\right) \geqslant \rho(c)+1=2$.

Assume next that for $\sigma \in G-\{1\}$, the $v(h-\sigma(h))$ 's are not equal. Let $c^{\prime}$ be the smallest jump of the ramification filtration of $G$ and let $\sharp\left(G^{c^{\prime}+}\right)=p^{n^{\prime}}$ for some integer $n^{\prime}<n$. Let $\varsigma_{1}=1, \varsigma_{2}, \ldots, \varsigma_{p^{n-n^{\prime}}}$ be liftings of all the elements of $G / G^{c^{\prime}+}$ in $G$. Observe that for any $\varsigma \in G-G^{c^{\prime}+}$ and $\sigma \in G^{c^{\prime}+}$, we have $u_{\varsigma \sigma}=u_{\varsigma}$. Hence

$$
\sum_{\varsigma \in G-G^{c^{\prime}+}} u_{\varsigma}=\sum_{j=2}^{p^{n-n^{\prime}}} \sum_{\sigma \in G^{c^{\prime}+}} u_{\varsigma_{j}}=p^{n^{\prime}} \sum_{j=2}^{p^{n-n^{\prime}}} u_{\varsigma_{j}}=0 .
$$

Hence $v\left(\sum_{\sigma \in G-G^{c^{\prime}+}}(\sigma(h)-h)\right) \geqslant 2$. Meanwhile, $v\left(\sum_{\sigma \in G^{c^{\prime}+}}(\sigma(h)-h)\right) \geqslant 2$, hence we obtain the inequality $v\left(\sum_{\sigma \in G}(\sigma(h)-h)\right) \geqslant 2$.

PROPOSITION 5.7. The composition of the canonical homomorphisms (Theorem 4.11)

$$
\pi_{1}^{\mathrm{alg}}\left(\Theta_{\bar{F}, \log }^{(c)}\right) \rightarrow \mathrm{Gr}_{\log }^{c} G_{K} \rightarrow G^{c}
$$

factors through $\pi_{1}^{\mathrm{alg}}\left(\Xi_{\bar{F}}^{(c)}\right)$ (4.10.4). In particular, for any non-trivial character $\chi: G^{c} \rightarrow$ $\mathbb{F}_{p}$, we have $\operatorname{rsw}(\chi) \in \Omega_{F}^{1} \otimes_{F} \mathfrak{m}_{\bar{K}}^{-c} / \mathfrak{m}_{\bar{K}}^{-c+}$.

The proof of this proposition is given in Subsection 9.3. 
5.8. For a non-trivial character $\chi: G^{c} \rightarrow \mathbb{F}_{p}$, we denote by $\bar{f}_{c, \chi}(T)$ the polynomial (Subsection 5.3)

$$
\bar{f}_{c, \chi}(T)=\prod_{\sigma \in \operatorname{ker} \chi}\left(T+u_{\sigma}\right) \in \bar{F}[T],
$$

and by $\tau \in G^{c}$ a lifting of $1 \in \mathbb{F}_{p}$. Recall that $\bar{f}_{c, \chi}$ is an additive polynomial with a non-zero linear term (Lemma 5.5), and that $\bar{f}_{c, \chi}\left(u_{\tau}\right)$ is independent of the choice of $\tau$.

THEOREM 5.9. For any non-trivial character $\chi: G^{c} \rightarrow \mathbb{F}_{p}$, the refined Swan conductor $\operatorname{rsw}(\chi)$ is given by

$$
\operatorname{rsw}(\chi)=-\mathrm{d} \bar{a}_{0} \otimes \frac{\pi^{-c}}{\left(\prod_{\sigma \in G-G^{c}} u_{\sigma}\right) \bar{f}_{c, \chi}^{p}\left(u_{\tau}\right)} \in \Omega_{F}^{1} \otimes_{F} \mathfrak{m}_{\bar{K}}^{-c} / \mathfrak{m}_{\bar{K}}^{-c+} .
$$

The proof of this theorem is given in Subsection 9.4.

COROLlARY 5.10. Let $M$ be a finite dimensional $\Lambda$-vector space with a non-trivial linear $G$-action. Then, with the notation of Subsection 4.6, we have (4.12.1)

$$
\mathrm{CC}_{\psi_{0}}(M) \in\left(\Omega_{F}^{1} \otimes_{F} \bar{F}\right)^{\otimes r},
$$

where $r=\operatorname{dim}_{\Lambda} M / M^{(0)}($ Lemma 4.5$)$.

\section{Tubular neighborhoods and normalized integral models.}

6.1. Let $R$ be an $\mathcal{O}_{K}$-algebra. Following ([AS2, 1]), we say that $R$ is formally of finite type over $\mathcal{O}_{K}$ if it is semi-local with radical $\mathfrak{m}_{R}, \mathfrak{m}_{R}$-adically complete, Noetherian and if the quotient $R / \mathfrak{m}_{R}$ is of finite type over $F$. We say that $R$ is topologically of finite type over $\mathcal{O}_{K}$ if it is $\pi$-adically complete, Noetherian and if the quotient $R / \pi R$ is of finite type over $F$.

6.2. We denote by $\mathrm{AFS}_{\mathcal{O}_{K}}$ the category of affine Noetherian adic formal schemes $\mathfrak{X}$ over $\operatorname{Spf}\left(\mathcal{O}_{K}\right)$ such that the closed sub-scheme $\mathfrak{X}_{\text {red }}$ defined by the largest ideal of definition of $\mathfrak{X}$, is a scheme of finite type over $\operatorname{Spec}(F)$. Let $A$ be a finite flat algebra over $\mathcal{O}_{K}$, and $i: \operatorname{Spf}(A) \rightarrow \mathfrak{X}$ a closed immersion in $\operatorname{AFS}_{\mathcal{O}_{K}}$. For any rational number $r>0$, following ([deJ, 7.1] and [AM, 2.1]), we associate to $i$ a $K$-affinoid variety $X^{r}$, called the tubular neighborhood of $i$ of thickening $r$, as follows. Let $\mathfrak{X}=\operatorname{Spf}(\mathcal{A}), I$ be the ideal of $\mathcal{A}$ which defines the immersion $i$ and $t, s>0$ be two integer such that $r=t / s$. Let $\mathcal{A}\left\langle I^{s} / \pi^{t}\right\rangle$ be the $\pi$-adic completion of the subalgebra of $\mathcal{A} \otimes \mathcal{O}_{K} K$ generated by $\mathcal{A}$ and $f / \pi^{t}$ for $f \in I^{s}$. Then $\mathcal{A}\left\langle I^{s} / \pi^{t}\right\rangle \otimes_{\mathcal{O}_{K}} K$ is a $K$-affinoid algebra which depends only on $r$. We denote by $X^{r}$ the $K$-affinoid variety $\operatorname{Sp}\left(\mathcal{A}\left\langle I^{s} / \pi^{t}\right\rangle \otimes_{\mathcal{O}_{K}} K\right)$. For rational numbers $r^{\prime}>r>0$, there exists a canonical morphism $X^{r^{\prime}} \rightarrow X^{r}$ which makes $X^{r^{\prime}}$ a rational sub-domain of $X^{r}$. The admissible union of the affinoid spaces $X^{r}$ for $r \in \mathbb{Q} \geqslant 0$ is a quasi-separated rigid variety over $K$.

PROPOSITION 6.3 (Finiteness theorem of Grauert-Remmert, [BGR, 6.4.1/3], [AS1, 4.2]). Let $\mathcal{R}$ be a geometrically reduced $K$-affinoid algebra. Then, there exists a finite separable extension $K^{\prime}$ of $K$ such that the supremum norm unit ball ([BGR, 3.8.1])

$$
\mathcal{R}_{\mathcal{O}_{K^{\prime}}}=\left\{f \in \mathcal{R} \otimes_{K} K^{\prime} ;|f|_{\text {sup }} \leqslant 1\right\} \subseteq \mathcal{R} \otimes_{K} K^{\prime}
$$


has a reduced geometric closed fiber $\mathcal{R}_{\mathcal{O}_{K^{\prime}}} \otimes_{\mathcal{O}_{K^{\prime}}} \bar{F}$. Moreover, the formation of $\mathcal{R}_{\mathcal{O}_{K^{\prime}}}$ commutes with any finite extension of $K^{\prime}$.

6.4. Let $\mathcal{R}$ be a geometrically reduced $K$-affinoid algebra. We consider the collection of $\mathcal{O}_{K^{\prime}}$-formal scheme $\operatorname{Spf}\left(\mathcal{R}_{\mathcal{O}_{K^{\prime}}}\right)$, where $K^{\prime}$ and $\mathcal{R}_{\mathcal{O}_{K^{\prime}}}$ are as in Proposition 6.3, as a unique model of $\operatorname{Sp}(\mathcal{R})$ over $\mathcal{O}_{\bar{K}}$. We call it the normalized integral model over $\mathcal{O}_{\bar{K}}$. We say that the normalized integral model of $\operatorname{Sp}(\mathcal{R})$ is defined over $K^{\prime}$ if the supremum norm unit ball $\mathcal{R}_{\mathcal{O}_{K^{\prime}}}$ has a reduced geometric special fiber. We call this reduced geometric special fiber over $\bar{F}$ the special fiber of the normalized integral model of $\operatorname{Sp}(\mathcal{R})$ over $\mathcal{O}_{\bar{K}}$.

PROPOSITION 6.5 ([AS1, 4.4]). Let $X$ be a geometrically reduced affinoid variety over $K, \mathfrak{X}$ its normalized integral model over $\mathcal{O}_{\bar{K}}$ and $\overline{\mathfrak{X}}$ the special fiber of $\mathfrak{X}$. Then the set of geometric connected components of $X$ and $\overline{\mathfrak{X}}$ are isomorphic.

6.6. Let $X$ be a geometrically reduced affinoid variety over $K, \mathfrak{X}$ its normalized integral model over $\mathcal{O}_{\bar{K}}$ and $\overline{\mathfrak{X}}$ the special fiber of $\mathfrak{X}$. If $\mathfrak{X}$ is defined over a finite Galois extension $K^{\prime}$ of $K$, we denote by $\mathfrak{X}_{\mathcal{O}_{K^{\prime}}}$ the normalized integral model of $X$ over $\mathcal{O}_{K^{\prime}}$. The natural $K^{\prime}$-semilinear action of $G_{K}$ on $X \otimes_{K} K^{\prime}$ extends to an $\mathcal{O}_{K^{\prime}}$-semi-linear action of $G_{K}$ on $\mathfrak{X}_{\mathcal{O}_{K^{\prime}}}$. If $K^{\prime \prime}$ is another finite Galois extension of $K$ containing $K^{\prime}$, then $\mathfrak{X}_{\mathcal{O}_{K^{\prime \prime}}^{\prime}}=\mathfrak{X}_{\mathcal{O}_{K^{\prime}}} \otimes_{\mathcal{O}_{K^{\prime}}} \mathcal{O}_{K^{\prime \prime}}$ and the semi-linear action of $G_{K}$ on both sides are compatible. Hence, it induces an $\bar{F}$-semi-linear action of $G_{K}$ on the special fiber $\overline{\mathfrak{X}}$, called the geometric monodromy ([AS1, 4.5]).

\section{Isogenies associated to extensions of type (II): the equal characteristic case.}

7.1. In this section, we assume that $K$ has characteristic $p$ and that the residue field $F$ of $\mathcal{O}_{K}$ is of finite type over a perfect field $F_{0}$. For an object $L$ of FÉ $/ K$ and an integer $r \geqslant 1$, we denote by $\left(\mathcal{O}_{L} / \mathfrak{m}_{L}^{r}\right) \widehat{\otimes}_{F_{0}} \mathcal{O}_{K}$ the completion of $\left(\mathcal{O}_{L} / \mathfrak{m}_{L}^{r}\right) \otimes_{F_{0}} \mathcal{O}_{K}$ relatively to the kernel of the homomorphism

$$
\left(\mathcal{O}_{L} / \mathfrak{m}_{L}^{r}\right) \otimes_{F_{0}} \mathcal{O}_{K} \rightarrow \mathcal{O}_{L} / \mathfrak{m}_{L}^{r}, \quad a \otimes b \mapsto a b,
$$

and by $\mathcal{O}_{L} \widehat{\widehat{\otimes}}_{F_{0}} \mathcal{O}_{K}$ the projective limit

$$
\lim _{r}\left(\mathcal{O}_{L} / \mathfrak{m}_{L}^{r}\right) \widehat{\otimes}_{F_{0}} \mathcal{O}_{K}
$$

We will always consider $\mathcal{O}_{L} \widehat{\widehat{\otimes}}_{F_{0}} \mathcal{O}_{K}$ as an $\mathcal{O}_{K}$-algebra by the homomorphism

$$
\mathcal{O}_{K} \rightarrow \mathcal{O}_{L} \widehat{\widehat{\otimes}}_{F_{0}} \mathcal{O}_{K}, \quad u \mapsto 1 \otimes u,
$$

(in the following, we always abbreviate $1 \otimes u$ by $u$ ) and we will consider it as an $\mathcal{O}_{L}$-algebra by

$$
\mathcal{O}_{L} \rightarrow \mathcal{O}_{L} \widehat{\widehat{\otimes}}_{F_{0}} \mathcal{O}_{K}, \quad v \mapsto v \otimes 1 .
$$

There is a canonical surjective homomorphism

$$
\mathcal{O}_{L} \widehat{\otimes}_{F_{0}} \mathcal{O}_{K} \rightarrow \mathcal{O}_{L}
$$

induced by the surjections (7.1.1). We denote by $I_{L}$ its kernel. 
PROPOSITION 7.2 ([AS2, 2.3]). Let $L$ be an object of $F E_{/ K}$.

(i) The $\mathcal{O}_{K}$-algebra $\mathcal{O}_{L} \widehat{\widehat{\otimes}}_{F_{0}} \mathcal{O}_{K}$ is formally of finite type and formally smooth over $\mathcal{O}_{K}$

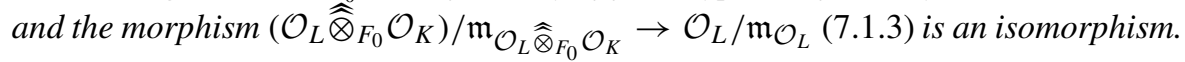

(ii) Any morphism $L \rightarrow L^{\prime}$ of $F E_{/ K}$ induces an isomorphism

$$
\mathcal{O}_{L^{\prime}} \otimes_{\mathcal{O}_{L}}\left(\mathcal{O}_{L} \widehat{\widehat{\otimes}}_{F_{0}} \mathcal{O}_{K}\right) \stackrel{\sim}{\rightarrow} \mathcal{O}_{L^{\prime}} \widehat{\widehat{\otimes}}_{F_{0}} \mathcal{O}_{K}
$$

7.3. Let $L$ be an object of FÉ $/ K$. By Proposition 7.2, $\operatorname{Spf}\left(\mathcal{O}_{L} \widehat{\widehat{\otimes}}_{F_{0}} \mathcal{O}_{K}\right)$ is an object of $\mathrm{AFS}_{\mathcal{O}_{K}}$ (Subsection 6.2). For any rational number $r>0$ and integer numbers $s, t>0$ such that $r=t / s$, we denote by $\mathcal{R}_{L}^{r}$ the $K$-affinoid algebra

$$
\mathcal{R}_{L}^{r}=\left(\mathcal{O}_{L} \widehat{\otimes}_{F_{0}} \mathcal{O}_{K}\right)\left\langle I_{L}^{s} / \pi^{t}\right\rangle \otimes_{\mathcal{O}_{K}} K,
$$

by $X_{L}^{r}=\operatorname{Sp}\left(\mathcal{R}_{L}^{r}\right)$ the tubular neighborhood of thickening $r$ of the closed immersion $\operatorname{Spf}\left(\mathcal{O}_{L}\right) \rightarrow \operatorname{Spf}\left(\mathcal{O}_{L} \widehat{\widehat{\otimes}}_{F_{0}} \mathcal{O}_{K}\right)$ (7.1.3), (Subsection 6.2), which is smooth over $K$ ([AS2, 1.7]). By Proposition 6.3, there exists a finite separable extension $K^{\prime}$ of $K$ such that the normalized integral model of $X_{L}^{r}$ over $\mathcal{O}_{\bar{K}}$ is defined over $K^{\prime}$ (Subsection 6.4). We denote by $\mathcal{R}_{L, \mathcal{O}_{K^{\prime}}}^{r}$ the supremum norm unit ball of $\mathcal{R}_{L}^{r} \otimes_{K} K^{\prime}$ (6.3.1), by $\mathfrak{X}_{L}^{r}$ the normalized integral model of $X_{L}^{r}$ over $\mathcal{O}_{\bar{K}}$ and by $\overline{\mathfrak{X}}_{L}^{r}$ the special fiber of $\mathfrak{X}_{L}^{r}$ (Subsection 6.4).

7.4. Let $m$ be the dimension of the $F$-vector space $\Omega_{F}^{1}$, which is finite. By ([AS2, 1.14.3]), there is an isomorphism of $\mathcal{O}_{K}$-algebras

$$
\mathcal{O}_{K}\left[\left[T_{0}, \ldots, T_{m}\right]\right] \stackrel{\sim}{\rightarrow} \mathcal{O}_{K} \widehat{\widehat{\otimes}}_{F_{0}} \mathcal{O}_{K},
$$

such that the composition of it and (7.1.3) $\mathcal{O}_{K}\left[\left[T_{0}, \ldots, T_{m}\right]\right] \stackrel{\sim}{\rightarrow} \mathcal{O}_{K} \widehat{\widehat{\otimes}}_{F_{0}} \mathcal{O}_{K} \rightarrow \mathcal{O}_{K}$ maps $T_{i}$ to 0 . Here the $\mathcal{O}_{K}$-algebra structure of $\mathcal{O}_{K} \widehat{\otimes}_{F_{0}} \mathcal{O}_{K}$ is as in (7.1.2). If $r$ is an integer $\geqslant 1$, we have an isomorphism of $K$-affinoid algebras

$$
K\left\langle T_{0} / \pi^{r}, \ldots, T_{m} / \pi^{r}\right\rangle \stackrel{\sim}{\rightarrow} \mathcal{R}_{K}^{r} .
$$

The normalized integral model $\mathfrak{X}_{K}^{r}$ is defined over $\mathcal{O}_{K}$, and we have an isomorphism

$$
\mathcal{O}_{K}\left\langle T_{0} / \pi^{r}, \ldots, T_{m} / \pi^{r}\right\rangle \stackrel{\sim}{\rightarrow}\left(\mathcal{O}_{K} \widehat{\widehat{\otimes}}_{F_{0}} \mathcal{O}_{K}\right)\left\langle I_{K} / \pi^{r}\right\rangle=\mathcal{R}_{K, \mathcal{O}_{K}}^{r} .
$$

Hence the closed fiber $\overline{\mathfrak{X}}_{K}^{r}$ is isomorphic to the affine scheme

$$
\operatorname{Spec} \bar{F}\left[T_{0} / \pi^{r}, \ldots, T_{m} / \pi^{r}\right] \text {. }
$$

In general, for any rational number $r>0$, the $K$-affinoid variety $X_{K}^{r}$ is isomorphic to $D^{m+1,(r)}$ and the rigid space $X_{K}=\cup_{r>0} X_{K}^{r}$ is isomorphic to $\stackrel{\circ}{D}^{m+1}$ (Subsection 5.2).

By ([AS2, 1.13, 2.4]), for any rational number $r>0$, there exists a canonical isomorphism $\overline{\mathfrak{X}}_{K}^{r} \stackrel{\sim}{\rightarrow} \Theta_{\bar{F}}^{(r)}$ (4.10.5) which is compatible with the geometric monodromy on $\overline{\mathfrak{X}}_{K}^{r}$ and the natural $G_{K}$-action on $\Theta_{\bar{F}}^{(r)}$ (via its action on $\mathfrak{m}_{\bar{K}}^{r} / \mathfrak{m}_{\bar{K}}^{r+}$ ). If $r$ is an integer, it is constructed as follows. Firstly, we have a natural ring isomorphism

$$
\bigoplus_{i=0}^{\infty} I_{K}^{i} / I_{K}^{i+1} \otimes_{\mathcal{O}_{K}} \mathfrak{m}_{K}^{-i r} / \mathfrak{m}_{K}^{-i r+1} \rightarrow \mathcal{R}_{K, \mathcal{O}_{K}}^{r} / \mathfrak{m}_{K} \mathcal{R}_{K, \mathcal{O}_{K}}^{r}, \quad \bar{b} \otimes \bar{c} \mapsto \overline{b c},
$$


by (7.4.1) and (7.4.3). Extending scalars, we have

$$
\overline{\mathfrak{X}}_{K}^{r} \stackrel{\sim}{\rightarrow} \operatorname{Spec}\left(\bigoplus_{i=0}^{\infty} I_{K}^{i} / I_{K}^{i+1} \otimes_{\mathcal{O}_{K}} \mathfrak{m}_{K}^{-i r} / \mathfrak{m}_{\bar{K}}^{-i r+}\right) .
$$

Then, from ([AS2, 1.14.3, 2.4]), we have an isomorphism of free $\mathcal{O}_{K}$-modules

$$
\widehat{\Omega}_{\mathcal{O}_{K} / F_{0}}^{1} \rightarrow I_{K} / I_{K}^{2}, \quad \mathrm{~d} t \mapsto \overline{1 \otimes t-t \otimes 1},
$$

which induces the isomorphism $\overline{\mathfrak{X}}_{K}^{r} \rightarrow \Theta_{\bar{F}}^{(r)}$.

7.5. Let $L$ be a finite Galois extension of $K$ of group $G$ and conductor $r>1$. By ([AS1, 7.2]), the natural action of $G$ on $\mathcal{O}_{L} \widehat{\widehat{\otimes}}_{F_{0}} \mathcal{O}_{K}$ induces an $\mathcal{O}_{\bar{K}}$-linear action of $G$ on $\mathfrak{X}_{L}^{r}$ making it an étale $G$-torsor over $\mathfrak{X}_{K}^{r}$. In particular, $X_{L}^{r}$ and $\overline{\mathfrak{X}}_{L}^{r}$ are étale $G$-torsors of $X_{K}^{r}$ and $\overline{\mathfrak{X}}_{K}^{r}$, respectively. The geometric monodromy action of $G_{K}$ on $\overline{\mathfrak{X}}_{L}^{r}$ (Subsection 6.6) commutes with the action of $G$. Let $\overline{\mathfrak{X}}_{L, 0}^{r}$ be a connected component of $\overline{\mathfrak{X}}_{L}^{r}$. The stabilizers of $\overline{\mathfrak{X}}_{L, 0}^{r}$ via these two actions are $G^{r}$ and $G_{K}^{r}$, respectively. Then, we get an isomorphism $G^{r} \stackrel{\sim}{\rightarrow}$ $\operatorname{Aut}\left(\overline{\mathfrak{X}}_{L, 0}^{r} / \overline{\mathfrak{X}}_{K}^{r}\right)$ and a surjection $G_{K}^{r} \rightarrow \operatorname{Aut}\left(\overline{\mathfrak{X}}_{L, 0}^{r} / \overline{\mathfrak{X}}_{K}^{r}\right)$ which implies that $G^{r}$ is commutative (cf. [AS2, 2.15.1]). Composing with $\overline{\mathfrak{X}}_{K}^{r} \stackrel{\sim}{\rightarrow} \Theta \bar{F}_{\bar{F}}^{(r)}$, the étale covering $\overline{\mathfrak{X}}_{L, 0}^{r} \rightarrow \Theta \frac{(r)}{F}$ induces a surjective homomorphism ([AS2, 2.15.1])

$$
\pi_{1}^{\mathrm{ab}}\left(\Theta_{\bar{F}}^{(r)}\right) \rightarrow \mathrm{Gr}^{r} G_{K} \rightarrow G^{r} .
$$

7.6. In the rest of this section, let $L / K$ be a finite Galois extension of type (II) and we take again the notation and assumptions of Subsections 5.1 and 5.2. By (7.2.1) and the proof of ([AS2, 1.6]), for any rational number $r>0$, we have an isomorphism

$$
\mathcal{R}_{K}^{r} \otimes_{\mathcal{O}_{K} \widehat{\otimes}_{F_{0}} \mathcal{O}_{K}}\left(\mathcal{O}_{L} \widehat{\widehat{\otimes}}_{F_{0}} \mathcal{O}_{K}\right) \stackrel{\sim}{\rightarrow} \mathcal{R}_{L}^{r}
$$

It induces, for any rational numbers $r>r^{\prime}>0$, an isomorphism

$$
\mathcal{R}_{K}^{r} \otimes_{\mathcal{R}_{K}^{r^{\prime}}} \mathcal{R}_{L}^{r^{\prime}} \stackrel{\sim}{\rightarrow} \mathcal{R}_{L}^{r},
$$

which gives a Cartesian diagram of rigid spaces

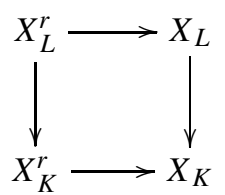

where $X_{K}=\bigcup_{r>0} X_{K}^{r}$ and $X_{L}=\bigcup_{r>0} X_{L}^{r}$.

We put

$$
\mathbb{f}(T)=T^{p^{n}}+\left(a_{p^{n}-1} \otimes 1\right) T^{p^{n}-1}+\cdots+\left(a_{0} \otimes 1\right) \in\left(\mathcal{O}_{K} \widehat{\widehat{\otimes}}_{F_{0}} \mathcal{O}_{K}\right)[T] .
$$

From (7.2.1) and (7.6.1), we have a surjection

$$
\tau_{L}: \mathcal{R}_{K}^{r}\langle T\rangle \rightarrow \mathcal{R}_{L}^{r}, \quad T \mapsto h \otimes 1,
$$


which induces an isomorphism that we denote abusively also by

$$
\tau_{L}: \mathcal{R}_{K}^{r}\langle T\rangle / \mathbb{f}(T) \stackrel{\sim}{\rightarrow} \mathcal{R}_{L}^{r} .
$$

In other terms, we have a co-Cartesian diagram of homomorphisms of $\mathcal{R}_{K}^{r}$-algebras

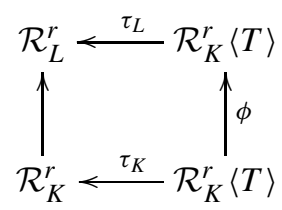

where $\phi(T)=\mathbb{f}(T)$ and $\tau_{K}(T)=0$. Hence, taking the union of the $K$-affinoid varieties associated to each of the $K$-affinoid algebras in (7.6.4) for $r \in \mathbb{Q}_{>0}$, we have a Cartesian diagram

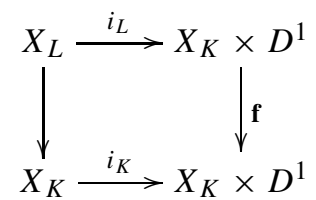

where $i_{L}$, f and $i_{K}$ are the morphisms induced by $\tau_{L}, \phi$ and $\tau_{K}$.

7.7. In the following, for any $0 \leqslant i \leqslant p^{n}-1$, we denote by $\alpha_{i}$ the element $a_{i}-a_{i} \otimes 1 \in$ $I_{K}$ (Subsection 7.1). When the conductor $c>2$, for each $1 \leqslant i \leqslant p^{n}-1, v\left(a_{i}\right) \geqslant 2$ (Lemma 5.6). Let $a_{i}^{\prime}=\pi^{-2} a_{i} \in \mathcal{O}_{K}$. We denote by $\alpha_{i}^{\prime}$ the element $a_{i}^{\prime}-a_{i}^{\prime} \otimes 1 \in I_{K}$ and by $\beta$ the element $\pi-\pi \otimes 1 \in I_{K}$. Then, we have

$$
\alpha_{i}=\left(a_{i}^{\prime}-\alpha_{i}^{\prime}\right)\left(2 \pi \beta-\beta^{2}\right)+\pi^{2} \alpha_{i}^{\prime} .
$$

Since $\alpha_{i}^{\prime}, \beta \in I_{K} \subset \pi^{c} \mathcal{R}_{K, \mathcal{O}_{K}}^{c}$, we have $\alpha_{i} \in \pi^{c+1} \mathcal{R}_{K, \mathcal{O}_{K}}^{c}$.

When $c=2$, we have $p=2, \sharp G=2, \rho(c)=1$ and $a_{1}^{\prime \prime}=\pi^{-1} a_{1} \in \mathcal{O}_{K}$. We denote by $\alpha_{1}^{\prime \prime}$ the element $a_{1}^{\prime \prime}-a_{1}^{\prime \prime} \otimes 1 \in I_{K}$. Then we have

$$
\alpha_{1}=\left(a_{1}^{\prime \prime}-\alpha_{1}^{\prime \prime}\right) \beta+\pi \alpha_{1}^{\prime \prime} .
$$

Since $\alpha_{1}^{\prime \prime}, \beta \in \pi^{c} \mathcal{R}_{K, \mathcal{O}_{K}}^{c}$, we have $\alpha_{1} \in \pi^{c} \mathcal{R}_{K, \mathcal{O}_{K}}^{c}$, and $\overline{\alpha_{1} / \pi^{c}}=\overline{a_{1}^{\prime \prime} \beta / \pi^{c}} \in \mathcal{R}_{K, \mathcal{O}_{K}}^{c} / \pi \mathcal{R}_{K, \mathcal{O}_{K}}^{c}$.

We put

$$
\mathbb{f}_{0}(T)=\sum_{0 \leqslant i \leqslant p^{n}-1}\left(\alpha_{i} / \pi^{c}\right) \cdot T^{i} \in \mathcal{R}_{K, \mathcal{O}_{K}}^{c}[T] .
$$

We have

$$
\mathbb{f}(T)=f(T)-\sum_{0 \leqslant i \leqslant p^{n}-1} \alpha_{i} T^{i}=f(T)-\pi^{c} \mathbb{f}_{0}(T) .
$$

In the rest of this section, we fix an embedding $L \rightarrow \bar{K}$. Recall that we put $\sharp\left(G^{c}\right)=p^{s}$ (Subsection 5.1). 
Proposition 7.8. The $K$-affinoid $X_{L}^{c}$ has $\sharp\left(G / G^{c}\right)=p^{n-s}$ geometric connected components. Let $\sigma_{1}, \ldots, \sigma_{p^{n-s}}$ be liftings of all the elements of $G / G^{c}$ in $G$. We have

$$
i_{L}\left(X_{L}^{c}\right) \subseteq \coprod_{1 \leqslant j \leqslant p^{n-s}} X_{K}^{c} \times\left(\sigma_{j}(h)+D^{1,(\rho(c))}\right) \subseteq X_{K} \times D^{1}
$$

and each disc of the disjoint union contains exact one geometric connected component of $X_{L}^{c}$.

Proof. By the Cartesian diagrams (7.6.2) and (7.6.5), we have

$$
i_{L}\left(X_{L}^{c}\right)=\mathbf{f}^{-1}\left(i_{K}\left(X^{c}\right)\right) \subseteq X_{K}^{c} \times D^{1} \subseteq X_{K} \times D^{1} .
$$

Taking in account the isomorphisms (7.4.2) and (7.4.3), for any point

$$
\left(t_{0}, \ldots, t_{m}, t\right) \in X_{K}^{c} \times D^{1}-\coprod_{1 \leqslant k \leqslant p^{n-s}} X_{K}^{c} \times\left(\sigma_{k}(h)+D^{1,(\rho(c))}\right),
$$

we have $v(f(t))<c$ and $v\left(\left(\alpha_{i} / \pi^{c}\right)\left(t_{0}, \ldots, t_{m}\right) t^{i}\right) \geqslant 0$. Hence $v\left(f(t)-\pi^{c} \mathbb{f}_{0}\left(t_{0}, \ldots, t_{m}, t\right)\right)$ $<c$ which means $\mathbf{f}\left(t_{0}, \ldots, t_{m}, t\right)=\left(t_{1}, \ldots, t_{m}, \mathbb{f}\left(t_{0}, \ldots, t_{m}, t\right)\right) \notin i_{K}\left(X_{K}^{c}\right)$. Thus (7.8.1) holds. By the proof of ([AS2, 2.15]), $X_{L}^{c}$ has exactly $p^{n-s}$ geometric connected components. Moreover, for any $1 \leqslant j \leqslant p^{n-s}, f\left(\sigma_{j}(h)\right)-\pi^{c} \mathbb{f}_{0}\left(0, \ldots, 0, \sigma_{j}(h)\right)=0$, hence each disc $X_{K}^{c} \times\left(\sigma_{j}(h)+D^{1,(\rho(c))}\right)$ contains at least one geometric connected component of $X_{L}^{c}$.

In the following, we denote by $\overline{\mathfrak{X}}_{L, 0}^{c}$ the connected component of $\overline{\mathfrak{X}}_{L}^{c}$ corresponding to the connected component $X_{L, 0}^{c}$ of $X_{L}^{c}$ containing $(0, \ldots, 0, h) \in X_{K}^{c} \times D^{1}$ defined over $L$.

Proposition 7.9. There exists a canonical Cartesian diagram

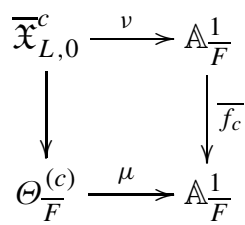

where $\overline{f_{c}}$ is defined in (5.4.1), such that if $\xi$ is the canonical coordinate of $\mathbb{A} \frac{1}{F}$, we have

$$
\mu^{*}(\xi)= \begin{cases}\mathrm{d} a_{0} \otimes \pi^{-c}, & \text { if } c>2, \\ \left(a_{1}^{\prime \prime} h \mathrm{~d} \pi+\mathrm{d} a_{0}\right) \otimes \pi^{-2}, & \text { if } c=2 .\end{cases}
$$

Moreover, for any $\sigma \in G^{c}$, the following diagram

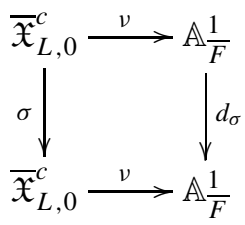

where $d_{\sigma}^{*}(\xi)=\xi-u_{\sigma}($ Subsection 5.3), is commutative. 
Proof. We consider the $K$-affinoid algebra $\mathcal{R}_{K}^{c}$ (resp. $\mathcal{R}_{L}^{c}$ ) as a sub-ring of the $L$ affinoid algebra $\mathcal{R}_{K}^{c} \otimes_{K} L$ (resp. $\mathcal{R}_{L}^{c} \otimes_{K} L$ ). By Proposition 7.8, we have

$$
X_{L, 0}^{c}=i_{L}^{-1}\left(X_{K}^{c} \times\left(h+D^{1,(\rho(c))}\right)\right) \cap X_{L}^{c} .
$$

Hence $X_{L, 0}^{c}$ is presented by the $L$-affinoid algebra

$$
\left(\mathcal{R}_{L}^{c} \otimes_{K} L\right)\left\langle T^{\prime}\right\rangle /\left(\pi^{\rho(c)} T^{\prime}+h-h \otimes 1\right) .
$$

By the isomorphism (7.6.3), (7.9.3) is isomorphic to

$$
\left(\mathcal{R}_{K}^{c} \otimes_{K} L\right)\left\langle T, T^{\prime}\right\rangle /\left(\mathbb{H}(T), \pi^{\rho(c)} T^{\prime}+h-T\right),
$$

which, after eliminating $T$ by the relation $\pi^{\rho(c)} T^{\prime}+h-T=0$, is

$$
\left(\mathcal{R}_{K}^{c} \otimes_{K} L\right)\left\langle T^{\prime}\right\rangle /\left(\mathbb{H}\left(\pi^{\rho(c)} T^{\prime}+h\right)\right) .
$$

In both cases, by Proposition 5.4 and Subsection 7.7, we have

$$
\begin{gathered}
\mathbb{f}\left(\pi^{\rho(c)} T^{\prime}+h\right) / \pi^{c} \in \mathcal{R}_{K, \mathcal{O}_{L}}^{c}\left\langle T^{\prime}\right\rangle, \\
\mathbb{f}\left(\pi^{\rho(c)} T^{\prime}+h\right) / \pi^{c+1} \notin \mathcal{R}_{K, \mathcal{O}_{L}}^{c}\left\langle T^{\prime}\right\rangle .
\end{gathered}
$$

Then the image of $\mathcal{R}_{K, \mathcal{O}_{L}}^{c}\left\langle T^{\prime}\right\rangle$ by the canonical surjection

$$
\left(\mathcal{R}_{K}^{c} \otimes_{K} L\right)\left\langle T^{\prime}\right\rangle \rightarrow\left(\mathcal{R}_{K}^{c} \otimes_{K} L\right)\left\langle T^{\prime}\right\rangle /\left(\mathbb{f}\left(\pi^{\rho(c)} T^{\prime}+h\right)\right)
$$

is

$$
\mathcal{R}_{K, \mathcal{O}_{L}}^{c}\left\langle T^{\prime}\right\rangle /\left(\mathbb{H}\left(\pi^{\rho(c)} T^{\prime}+h\right) / \pi^{c}\right) .
$$

Extending the scalars from $\mathcal{O}_{L}$ to $\bar{F}$, we obtain the following $\bar{F}$-algebra:

(i) if $c>2$,

$$
\left(\mathcal{R}_{K, \mathcal{O}_{L}}^{c} \otimes_{\mathcal{O}_{L}} \bar{F}\right)\left[T^{\prime}\right] /\left(\overline{f_{c}}\left(T^{\prime}\right)-\overline{\alpha_{0} / \pi^{c}}\right)
$$

(ii) if $c=2$,

$$
\left(\mathcal{R}_{K, \mathcal{O}_{L}}^{c} \otimes_{\mathcal{O}_{L}} \bar{F}\right)\left[T^{\prime}\right] /\left(\overline{f_{2}}\left(T^{\prime}\right)-\overline{\left(\alpha_{0}+a_{1}^{\prime \prime} h \beta\right) / \pi^{2}}\right) .
$$

From isomorphisms (7.4.4), (7.4.6) and the canonical exact sequence (4.10.2), we know that when $c>2$ (resp. $c=2$ ), $\overline{\alpha_{0} / \pi^{c}}$ (resp. $\overline{\left(\alpha_{0}+a_{1}^{\prime \prime} h \beta\right) / \pi^{2}}$ ) is a non-zero linear term in $\bar{F} \otimes \mathcal{O}_{L} \mathcal{R}_{K, \mathcal{O}_{L}}^{c}$. Hence (7.9.6) and (7.9.7) are all reduced. Then, by ([AS1, 4.1]),

$$
\operatorname{Spf}\left(\mathcal{R}_{K, \mathcal{O}_{L}}^{c}\left\langle T^{\prime}\right\rangle /\left(\mathbb{H}\left(\pi^{\rho(c)} T^{\prime}+h\right) / \pi^{c}\right)\right)
$$

is the normalized integral model of $X_{K, 0}^{c}$ defined over $\mathcal{O}_{L}$. Hence $\overline{\mathfrak{X}}_{L, 0}^{c}$ is defined by the $\bar{F}$-algebra (7.9.6) (resp. (7.9.7)) when $c>2$ (resp. $c=2)$. We put

$$
v: \overline{\mathfrak{X}}_{L, 0}^{c} \rightarrow \mathbb{A} \frac{1}{F}=\operatorname{Spec}(\bar{F}[\xi]), \quad v^{*}(\xi)=T^{\prime} .
$$

It follows form the isomorphism $\overline{\mathfrak{X}}_{K}^{c} \stackrel{\sim}{\rightarrow} \Theta_{\bar{F}}^{(c)}$ (Subsection 7.4) that (7.9.1) is Cartesian.

For any $\sigma \in G^{c}$, let $y_{\sigma}(x)$ be a polynomial $b_{r} x^{r}+\cdots+b_{0} \in \mathcal{O}_{K}[x]$, where $r \leqslant p^{n}-1$, such that $y_{\sigma}(h)=(h-\sigma(h)) / \pi^{\rho(c)} \in \mathcal{O}_{L}$. We denote by $\mathrm{y}_{\sigma}$ the polynomial

$$
\mathbb{y}_{\sigma}(x)=\left(b_{r} \otimes 1\right) x^{r}+\cdots+\left(b_{0} \otimes 1\right) \in \mathcal{R}_{K}^{c}[x] .
$$


The action of $\sigma$ on $\mathcal{R}_{K}^{c}\langle T\rangle / \mathbb{f}(T)$ (isomorphic to $\mathcal{R}_{L}^{c}$ (7.6.3)) is given by : $T \mapsto T-\left(\pi^{\rho(c)} \otimes\right.$ 1) $\mathbb{y}(T)$. Hence the action of $\sigma$ on (7.9.4) is given by

$$
T^{\prime} \mapsto T^{\prime}-\mathbb{y}_{\sigma}\left(\pi^{\rho(c)} T^{\prime}+h\right)-\left(\left(\pi^{\rho(c)} \otimes 1-\pi^{\rho(c)}\right) / \pi^{\rho(c)}\right) \mathbb{y}_{\sigma}\left(\pi^{\rho(c)} T^{\prime}+h\right)
$$

and the induced action on (7.9.5) is given by the same formula. Since $\pi^{\rho(c)} \otimes 1-\pi^{\rho(c)} \in$ $\pi^{c} \mathcal{R}_{K, \mathcal{O}_{K}}^{c}$ and $c>\rho(c)$, the reduction of $\left(\pi^{\rho(c)} \otimes 1-\pi^{\rho(c)}\right) / \pi^{\rho(c)}$ to the geometric special fiber is 0 . For any $0 \leqslant j \leqslant r, b_{j} \otimes 1-b_{j} \in \pi^{c} \mathcal{R}_{K, \mathcal{O}_{K}}^{c}$. Then, the reduction of $\mathrm{y}_{\sigma}\left(\pi^{\rho(c)} T^{\prime}+h\right)$ to the geometric special fiber is (Subsection 5.3)

$$
\overline{\mathbb{Y}_{\sigma}\left(\pi^{\rho(c)} T^{\prime}+h\right)}=\overline{y_{\sigma}\left(\pi^{\rho(c)} T^{\prime}+h\right)}=\overline{y_{\sigma}(h)}=u_{\sigma} .
$$

Hence, diagram (7.9.2) is commutative.

\section{Isogenies associated to extensions of type (II): the unequal characteristic case.}

8.1. In this section, we assume that $K$ has characteristic 0 and that the residue field $F$ of $\mathcal{O}_{K}$ is of finite type over a perfect field $F_{0}$. Let $K_{0}$ be the fraction field of the ring of Witt vectors $W\left(F_{0}\right)=O_{K_{0}}$ considered as a subfield of $K$. We denote by $m$ the dimension of the $F$-vector space $\Omega_{F}^{1}$, which is finite.

8.2. Let $L$ be an object of $\mathrm{FE}_{/ K}$. We call an $\mathcal{O}_{K_{0}}$-presentation of Cartier type of $\mathcal{O}_{L}$ a pair $\left(\mathcal{A}_{L}, j: \mathcal{A}_{L} \rightarrow \mathcal{O}_{L}\right)$, where $\mathcal{A}_{L}$ is a complete semi-local Noetherian $\mathcal{O}_{K_{0}}$-algebra formally smooth of relative dimension $m+1$ over $\mathcal{O}_{K_{0}}$ and $j$ a surjective homomorphism of $\mathcal{O}_{K_{0}}$-algebra inducing an isomorphism $\mathcal{A}_{L} / \mathfrak{m}_{\mathcal{A}_{L}} \stackrel{\sim}{\rightarrow} \mathcal{O}_{L} / \mathfrak{m}_{L}$ such that the kernel of $j$ is generated by a non-zero divisor of $\mathcal{A}_{L}$.

Let $L_{1}, L_{2}$ be two objects of $\mathrm{FEE}_{/ K}$ and $\left(\mathcal{A}_{L_{1}}, j_{1}: \mathcal{A}_{L_{1}} \rightarrow \mathcal{O}_{L_{1}}\right),\left(\mathcal{A}_{L_{2}}, j_{2}: \mathcal{A}_{L_{2}} \rightarrow\right.$ $\left.\mathcal{O}_{L_{2}}\right)$ two $\mathcal{O}_{K_{0}}$-presentations of Cartier type. A morphism $(g, g)$ from $\left(\mathcal{A}_{L_{1}}, j_{1}\right)$ to $\left(\mathcal{A}_{L_{2}}, j_{2}\right)$ is a pair of $\mathcal{O}_{K_{0}}$-homomorphisms $g: \mathcal{O}_{L_{1}} \rightarrow \mathcal{O}_{L_{2}}$ and $g: \mathcal{A}_{L_{1}} \rightarrow \mathcal{A}_{L_{2}}$ such that the diagram

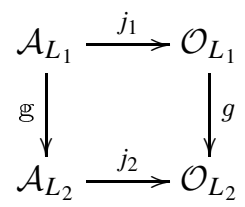

is commutative. We say that $(g, g)$ is finite and flat if $g$ is finite and flat and if the diagram (8.2.1) is co-Cartesian.

Proposition 8.3 ([AS2, 2.7, 2.8]).

(i) Any object of $F E_{/ K}$ admits an $\mathcal{O}_{K_{0}}$-presentation of Cartier type.

(ii) Let $g: L_{1} \rightarrow L_{2}$ be a morphism of $F E_{/ K}$, and $\left(\mathcal{A}_{L_{1}}, j_{1}\right),\left(\mathcal{A}_{L_{2}}, j_{2}\right)$ two $\mathcal{O}_{K_{0}}{ }^{-}$ presentations of Cartier type. Then there exist a morphism $\mathrm{g}: \mathcal{A}_{L_{1}} \rightarrow \mathcal{A}_{L_{2}}$ such that $(\mathrm{g}, \mathrm{g})$ is a morphism of $\mathcal{O}_{K_{0}}$-presentations of Cartier type.

(iii) Let $g: L_{1} \rightarrow L_{2}$ be a morphism of $F E_{/ K}$ and $(g, g)$ a morphism between $\mathcal{O}_{K_{0}}$ presentations of Cartier type $\left(\mathcal{A}_{L_{1}}, j_{1}\right)$ and $\left(\mathcal{A}_{L_{2}}, j_{2}\right)$. If a uniformizer $\pi_{0}$ of $K_{0}$ is not a uniformizer of any factor of $\mathcal{O}_{L_{1}}$, then $(\mathrm{g}, \mathrm{g})$ is finite and flat. 
8.4. Let $L$ be an object of $\mathrm{FE}_{/ K}$, and $\left(\mathcal{A}_{L}, j: \mathcal{A}_{L} \rightarrow \mathcal{O}_{L}\right)$ an $\mathcal{O}_{K_{0}}$-presentation of Cartier type. We denote by $\left(\mathcal{A}_{L} / \mathfrak{m}^{r} \mathcal{A}_{L}\right) \widehat{\otimes}_{\mathcal{O}_{K_{0}}} \mathcal{O}_{K}$ the formal completion of $\left(\mathcal{A}_{L} / \mathfrak{m}^{r} \mathcal{A}_{L}\right) \otimes_{\mathcal{O}_{K_{0}}}$ $\mathcal{O}_{K}$ relatively to the kernel of the homomorphism

$$
\left(\mathcal{A}_{L} / \mathfrak{m}_{\mathcal{A}_{L}}^{r}\right) \otimes_{\mathcal{O}_{K_{0}}} \mathcal{O}_{K} \rightarrow \mathcal{O}_{L} / \mathfrak{m}_{\mathcal{O}_{L}}^{r}, \quad a \otimes b \mapsto a b,
$$

and by $\mathcal{A}_{L} \widehat{\otimes}_{\mathcal{O}_{K_{0}}} \mathcal{O}_{K}$ the projective limit

$$
\mathcal{A}_{L} \widehat{\widehat{\otimes}}_{\mathcal{O}_{K_{0}}} \mathcal{O}_{K}=\underset{r}{\lim }\left(\left(\mathcal{A}_{L} / \mathfrak{m}_{\mathcal{A}_{L}}^{r}\right) \widehat{\otimes}_{\mathcal{O}_{K_{0}}} \mathcal{O}_{K}\right)
$$

We will always consider $\mathcal{A}_{L} \widehat{\widehat{\otimes}}_{\mathcal{O}_{K_{0}}} \mathcal{O}_{K}$ as an $\mathcal{O}_{K}$-algebra by the homomorphism

$$
\mathcal{O}_{K} \rightarrow \mathcal{A}_{L} \widehat{\otimes}_{\mathcal{O}_{K_{0}}} \mathcal{O}_{K}, \quad u \mapsto 1 \otimes u,
$$

(in the following, we always abbreviate $1 \otimes u$ by $u$ ) and we will consider it as an $\mathcal{A}_{L}$-algebra by

$$
\mathcal{A}_{L} \rightarrow \mathcal{A}_{L} \widehat{\widehat{\otimes}}_{\mathcal{O}_{K_{0}}} \mathcal{O}_{K}, \quad v \mapsto v \otimes 1
$$

There is a canonical surjective homomorphism

$$
\mathcal{A}_{L} \widehat{\widehat{\otimes}}_{\mathcal{O}_{K_{0}}} \mathcal{O}_{K} \rightarrow \mathcal{O}_{L}
$$

induced by the surjections (8.4.1). We denote by $I_{L}$ its kernel.

Proposition 8.5 ([AS2, 2.9]). Let $L$ be an object of $F E_{/ K}$, and $\left(\mathcal{A}_{L}, j: \mathcal{A}_{L} \rightarrow\right.$ $\mathcal{O}_{L}$ ) an $\mathcal{O}_{K_{0}}$-presentation of Cartier type. Then,

(i) The $\mathcal{O}_{K}$-algebra $\mathcal{A}_{L} \widehat{\widehat{\otimes}}_{\mathcal{O}_{K_{0}}} \mathcal{O}_{K}$ is formally of finite type and formally smooth over

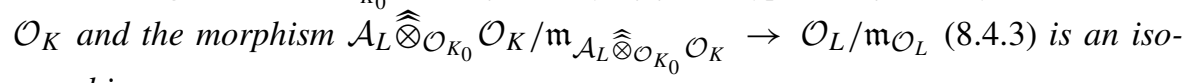
morphism.

(ii) Let $L^{\prime}$ be another object in $F E_{/ K}$ and $\left(\mathcal{A}_{L^{\prime}}, j^{\prime}: \mathcal{A}_{L^{\prime}} \rightarrow \mathcal{O}_{L^{\prime}}\right)$ an $\mathcal{O}_{K_{0}}$-presentation of Cartier type. If a uniformizer $\pi_{0}$ is not a uniformizer of any factor of $\mathcal{O}_{L}$, then, any morphism $\left(\mathcal{A}_{L}, j\right) \rightarrow\left(\mathcal{A}_{L^{\prime}}, j^{\prime}\right)$ induces an isomorphism

$$
\mathcal{A}_{L^{\prime}} \otimes_{\mathcal{A}_{L}}\left(\mathcal{A}_{L} \widehat{\widehat{\otimes}} \mathcal{O}_{K_{0}} \mathcal{O}_{K}\right) \stackrel{\sim}{\rightarrow} \mathcal{A}_{L^{\prime}} \widehat{\widehat{\otimes}}_{\mathcal{O}_{K_{0}}} \mathcal{O}_{K}
$$

PROOF. Part (i) is proved in ([AS2, 2.9]). For part (ii), we may assume $L$ and $L^{\prime}$ are fields. We denote by $e$ the ramification index of the extension $L^{\prime} / L$. For any integer $r \geqslant 1$, we have the following canonical commutative diagram

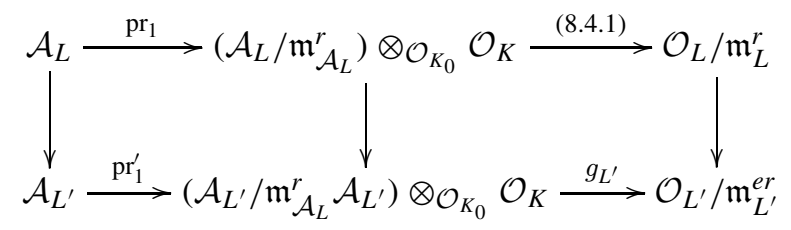

such that each square is co-Cartesian. We denote by $\left(\mathcal{A}_{L^{\prime}} / \mathfrak{m}^{r}{ }_{\mathcal{A}_{L}} \mathcal{A}_{L^{\prime}}\right) \widehat{\otimes}_{\mathcal{O}_{K_{0}}} \mathcal{O}_{K}$ the formal completion of $\left(\mathcal{A}_{L^{\prime}} / \mathfrak{m}^{r}{ }_{\mathcal{A}_{L}} \mathcal{A}_{L^{\prime}}\right) \otimes_{\mathcal{O}_{K}} \mathcal{O}_{K}$ relatively to the kernel of $g_{L^{\prime}}$. Since $\mathcal{A}_{L}$ is a Noetherian local ring, by proposition 8.3(iii) and Nakayama's lemma, $\mathcal{A}_{L^{\prime}}$ is a finite free $\mathcal{A}_{L}$-module. 
Then, we have

$$
\mathcal{A}_{L^{\prime}} \otimes_{\mathcal{A}_{L}}\left(\mathcal{A}_{L} / \mathfrak{m}_{\mathcal{A}_{L}}^{r}\right) \widehat{\otimes}_{\mathcal{O}_{K_{0}}} \mathcal{O}_{K} \stackrel{\sim}{\rightarrow}\left(\mathcal{A}_{L^{\prime}} / \mathfrak{m}_{\mathcal{A}_{L}}^{r} \mathcal{A}_{L^{\prime}}\right) \widehat{\otimes}_{\mathcal{O}_{K_{0}}} \mathcal{O}_{K}
$$

After taking projective limit on both sides, we obtain

$$
\mathcal{A}_{L^{\prime}} \otimes_{\mathcal{A}_{L}}\left(\mathcal{A}_{L} \widehat{\widehat{\otimes}}_{\mathcal{O}_{K_{0}}} \mathcal{O}_{K}\right) \stackrel{\sim}{\rightarrow} \underset{r}{\lim }\left(\left(\mathcal{A}_{L^{\prime}} / \mathfrak{m}_{\mathcal{A}_{L}}^{r} \mathcal{A}_{L^{\prime}}\right) \widehat{\otimes}_{\mathcal{O}_{K_{0}}} \mathcal{O}_{K}\right)
$$

By the proof of ([AS2, 2.7.3]), we obtain that $\mathfrak{m}_{\mathcal{A}_{L^{\prime}}}^{e} \subseteq \mathfrak{m}_{\mathcal{A}_{L}} \mathcal{A}_{L^{\prime}} \subseteq \mathfrak{m}_{\mathcal{A}_{L^{\prime}}}$. Hence, for any integer $r \geqslant 1$, we have two surjections

$$
\left(\mathcal{A}_{L^{\prime}} / \mathfrak{m}_{\mathcal{A}_{L^{\prime}}}^{e r}\right) \widehat{\otimes}_{\mathcal{O}_{K_{0}}} \mathcal{O}_{K} \rightarrow\left(\mathcal{A}_{L^{\prime}} / \mathfrak{m}_{\mathcal{A}_{L}}^{r} \mathcal{A}_{L^{\prime}}\right) \widehat{\otimes}_{\mathcal{O}_{K_{0}}} \mathcal{O}_{K} \rightarrow\left(\mathcal{A}_{L^{\prime}} / \mathfrak{m}_{\mathcal{A}_{L^{\prime}}}^{r}\right) \widehat{\otimes}_{\mathcal{O}_{K_{0}}} \mathcal{O}_{K},
$$

which imply

$$
\underset{r}{\lim }\left(\left(\mathcal{A}_{L^{\prime}} / \mathfrak{m}_{\mathcal{A}_{L}}^{r} \mathcal{A}_{L^{\prime}}\right) \widehat{\otimes}_{\mathcal{O}_{K_{0}}} \mathcal{O}_{K}\right) \stackrel{\sim}{\rightarrow} \mathcal{A}_{L^{\prime}} \widehat{\widehat{\otimes}}_{\mathcal{O}_{K_{0}}} \mathcal{O}_{K}
$$

Combining (8.5.2) and (8.5.3), we get (ii).

8.6. Let $L$ be an object of $\mathrm{FE}_{/ K}$, and $\left(\mathcal{A}_{L}, j: \mathcal{A}_{L} \rightarrow \mathcal{O}_{L}\right)$ an $\mathcal{O}_{K_{0}}$-presentation of Cartier type. We will introduce objects analogue of those defined in $\S 7$, and denote them by the same notation. For any rational number $r>0$ and integer numbers $s, t>0$ such that $r=t / s$, we denote by $\mathcal{R}_{L}^{r}$ the $K$-affinoid algebra

$$
\mathcal{R}_{L}^{r}=\left(\mathcal{A}_{L} \widehat{\widehat{\otimes}}_{\mathcal{O}_{K}} \mathcal{O}_{K}\right)\left\langle I_{K}^{s} / \pi^{t}\right\rangle \otimes_{\mathcal{O}_{K}} K,
$$

by $X_{L}^{r}=\operatorname{Sp}\left(\mathcal{R}_{L}^{r}\right)$ the tubular neighborhood of thickening $r$ of the immersion

$$
\operatorname{Spf}\left(\mathcal{O}_{L}\right) \rightarrow \operatorname{Spf}\left(\mathcal{A}_{L} \widehat{\otimes}_{\mathcal{O}_{K_{0}}} \mathcal{O}_{K}\right)
$$

which is smooth over $K$ ([AS2, 1.7]). By Proposition 6.3, there exists a finite separable extension $K^{\prime}$ of $K$ such that the normalized integral model of $X_{L}^{c}$ is defined over $K^{\prime}$ (Subsection 6.4). We denote by $\mathcal{R}_{L, \mathcal{O}_{K^{\prime}}}^{r}$ the supremum norm unit ball of $\mathcal{R}_{L}^{r} \otimes_{K} K^{\prime}$ (6.3.1), by $\mathfrak{X}_{L}^{r}$ the normalized integral model of $X_{L}^{r}$ over $\mathcal{O}_{\bar{K}}$ and by $\overline{\mathfrak{X}}_{L}^{r}$ the special fiber of $\mathfrak{X}_{L}^{r}$.

8.7. In the following of this section, we assume that $p$ is not a uniformizer of $K$. By ([AS2] 1.14.3), there is an isomorphism of $\mathcal{O}_{K}$-algebras

$$
\mathcal{O}_{K}\left[\left[T_{0}, \ldots, T_{m}\right]\right] \stackrel{\sim}{\rightarrow} \mathcal{A}_{K} \widehat{\widehat{\otimes}}_{\mathcal{O}_{K}} \mathcal{O}_{K},
$$

such that the composition of it and (8.4.3) $\mathcal{O}_{K}\left[\left[T_{0}, \ldots, T_{m}\right]\right] \rightarrow \mathcal{A}_{K} \widehat{\widehat{\otimes}}_{\mathcal{O}_{K}} \mathcal{O}_{K} \rightarrow \mathcal{O}_{K}$ maps $T_{i}$ to 0 . If $r$ is an integer $\geqslant 1$, we have an isomorphism of $K$-affinoid algebras

$$
K\left\langle T_{0} / \pi^{r}, \ldots, T_{m} / \pi^{r}\right\rangle \stackrel{\sim}{\rightarrow} \mathcal{R}_{K}^{r} .
$$

The normalized integral model $\mathfrak{X}_{K}^{r}$ is defined over $\mathcal{O}_{K}$, and we have an isomorphism

$$
\mathcal{O}_{K}\left\langle T_{0} / \pi^{r}, \ldots, T_{m} / \pi^{r}\right\rangle \stackrel{\sim}{\rightarrow}\left(\mathcal{A}_{K} \widehat{\widehat{\otimes}}_{\mathcal{O}_{K}} \mathcal{O}_{K}\right)\left\langle I_{K} / \pi^{r}\right\rangle=\mathcal{R}_{K, \mathcal{O}_{K}}^{r} .
$$

Hence the geometric closed fiber $\overline{\mathfrak{X}}_{K}^{r}$ is isomorphic to the affine scheme

$$
\operatorname{Spec} \bar{F}\left[T_{0} / \pi^{r}, \ldots, T_{m} / \pi^{r}\right] \text {. }
$$


In general, for any rational number $r>0$, the $K$-affinoid variety $X_{K}^{r}$ is isomorphic to $D^{m+1,(r)}$ and the rigid space $X_{K}=\bigcup_{r>0} X_{K}^{r}$ is isomorphic to $\stackrel{\circ}{D}^{m+1}$ (Subsection 5.2).

By ([AS2, 2.11.2]), we have an isomorphism

$$
\left(I_{K} / I_{K}^{2}\right) \otimes_{\mathcal{O}_{K}} F \rightarrow \widehat{\Omega}_{\mathcal{O}_{K} / \mathcal{O}_{K_{0}}}^{1} \otimes_{\mathcal{O}_{K}} F
$$

such that for any $x \in \mathcal{O}_{K}$ and $\widetilde{x}$ a lifting in $\mathcal{A}_{K}$, the image of $(\overline{1 \otimes x-\widetilde{x} \otimes 1}) \otimes 1$ is $\mathrm{d} x \otimes 1$. From ([AS2, 1.14.3, 2.11.2]), for any rational number $r>0$, the inverse of (8.7.4) gives an isomorphism $\overline{\mathfrak{X}}_{K}^{r} \stackrel{\sim}{\rightarrow} \Theta_{\bar{F}}^{(r)}$. When $r$ is an integer, the construction of the isomorphism is similar to the equal characteristic case (Subsection 7.4).

REMARK 8.8. From (8.7.4), we notice that for any element $\tilde{x} \in \operatorname{ker}\left(\mathcal{A}_{K} \rightarrow \mathcal{O}_{K}\right)$, the class $(\overline{\widetilde{x} \otimes 1}) \otimes 1$ vanishes in $\left(I_{K} / I_{K}^{2}\right) \otimes_{\mathcal{O}_{K}} F$. It is equivalent to say that $\tilde{x} \otimes 1 \in I_{K}^{2}+\pi I_{K}$.

8.9. Let $L$ be a finite Galois extension of $K$ of group $G$ and conductor $c>1$. Let $(g, \mathrm{~g})$ be a finite and flat morphism from $\left(\mathcal{A}_{K}, j_{K}: \mathcal{A}_{K} \rightarrow \mathcal{O}_{K}\right)$ to $\left(\mathcal{A}_{L}, j_{L}: \mathcal{A}_{L} \rightarrow \mathcal{O}_{L}\right)$ (Subsection 8.2). By (8.5.1), g induces a finite flat morphism g $\otimes$ id : $\mathcal{A}_{K} \widehat{\widehat{\otimes}}_{\mathcal{O}_{K_{0}}} \mathcal{O}_{K} \rightarrow$ $\mathcal{A}_{L} \widehat{\widehat{\otimes}}_{\mathcal{O}_{K_{0}}} \mathcal{O}_{K}$. Hence, for any rational number $r>0$, it gives a morphism of smooth $K$ affinoid varieties $X_{L}^{r} \rightarrow X_{K}^{r}$ ([AS2, 1.6]) which induces morphisms $\mathfrak{X}_{L}^{r} \rightarrow \mathfrak{X}_{K}^{r}$ and $\overline{\mathfrak{X}}_{K}^{r} \rightarrow$ $\overline{\mathfrak{X}}_{L}^{r}$. For any $\sigma \in G$, there is a morphism $g_{\sigma}$ making the following diagram commutative (Proposition 8.3(iii))

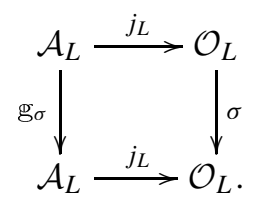

The pair $\left(\sigma, g_{\sigma}\right)$ induces automorphisms of $X_{L}^{r}, \mathfrak{X}_{L}^{r}$ and $\overline{\mathfrak{X}}_{L}^{r}$. Notice that, $\mathrm{g}_{\sigma}$ is not unique in general and may not be an $\mathcal{A}_{K}$-homomorphism. Hence the automorphisms of $X_{L}^{r}, \mathfrak{X}_{L}^{r}$ and $\overline{\mathfrak{X}}_{L}^{r}$ induced by all possible $g_{\sigma}$ may not be uniquely determined by $\sigma \in G$. Luckily, by ([AS2, 2.13]), the induced automorphism of $\overline{\mathfrak{X}}_{L}^{c}$ is $\overline{\mathfrak{X}}_{K}^{c}$-invariant and independent of the choice of $\mathrm{g}_{\sigma}$. Hence $\overline{\mathfrak{X}}_{L}^{c} \rightarrow \overline{\mathfrak{X}}_{K}^{c}$ is a finite étale $G$-torsor ([AS2, 1.16.2]). The geometric monodromy action of $G_{K}$ on $\overline{\mathfrak{X}}_{L}^{c}$ commutes with the action of $G$. Let $\overline{\mathfrak{X}}_{L, 0}^{c}$ be a connected component of $\overline{\mathfrak{X}}_{L}^{c}$. The stabilizers of $\overline{\mathfrak{X}}_{L, 0}^{c}$ via these two actions are $G^{c}$ and $G_{K}^{c}$, respectively ([AS2, 2.15.1]). Then, we get an isomorphism $G^{c} \stackrel{\sim}{\rightarrow} \operatorname{Aut}\left(\overline{\mathfrak{X}}_{L, 0}^{c} / \overline{\mathfrak{X}}_{K}^{c}\right)$ and a surjection $G_{K}^{c} \rightarrow \operatorname{Aut}\left(\overline{\mathfrak{X}}_{L, 0}^{c} / \overline{\mathfrak{X}}_{K}^{c}\right)$ which imply that $G^{c}$ is commutative (cf. [AS2, 2.15.1]). Composing with $\overline{\mathfrak{X}}_{K}^{r} \stackrel{\sim}{\rightarrow} \underset{\Theta^{\prime}}{\stackrel{(r)}{*}}$, the étale covering $\overline{\mathfrak{X}}_{L, 0}^{c} \rightarrow \Theta_{\bar{F}}^{(r)}$ induces a surjective homomorphism ([AS2, 2.15.1])

$$
\pi_{1}^{\mathrm{ab}}\left(\Theta_{\bar{F}}^{(r)}\right) \rightarrow \mathrm{Gr}^{c} G_{K} \rightarrow G^{c} .
$$

8.10. In the following of this section, we assume that the finite Galois extension $L / K$ is of type (II) and we take again the notation and assumptions of Subsections 5.1 and 5.2. Let $(g, g)$ be a finite and flat morphism as in Subsection 8.9. Let $\widetilde{h}$ be a lifting of $h \in \mathcal{O}_{L}$ in $\mathcal{A}_{L}$. 
Since $\mathcal{A}_{K}$ is a Noetherian local ring, by Proposition 8.3(iii) and Nakayama's lemma, we have that $\mathcal{A}_{L}$ is a finite free $\mathcal{A}_{K}$-module of rank $\sharp G$ and that $\mathcal{A}_{L}=\mathcal{A}_{K}[\tilde{h}]$. Let

$$
\tilde{f}(T)=T^{p^{n}}+\widetilde{a}_{p^{n}-1} T_{p^{n}-1}+\cdots+\widetilde{a}_{0} \in \mathcal{A}_{K}[T],
$$

be a lifting of $f[T] \in \mathcal{O}_{K}[T]$ such that $\tilde{h}$ is a zero. We have an isomorphism

$$
\mathcal{A}_{K}[T] /(\tilde{f}(T)) \stackrel{\sim}{\rightarrow} \mathcal{A}_{L}, \quad T \mapsto \widetilde{h} .
$$

By (8.5.1) and the proof of ([AS2, 1.6]), we have an isomorphism

$$
\mathcal{R}_{K}^{r} \otimes_{\mathcal{A}_{K} \widehat{\widehat{\otimes}}_{\mathcal{O}_{K_{0}}} \mathcal{O}_{K}}\left(\mathcal{A}_{L} \widehat{\widehat{\otimes}}_{\mathcal{O}_{K_{0}}} \mathcal{O}_{K}\right) \stackrel{\sim}{\rightarrow} \mathcal{R}_{L}^{r} .
$$

It induces, for any rational numbers $r>r^{\prime}>0$, an isomorphism

$$
\mathcal{R}_{K}^{r} \otimes_{\mathcal{R}_{K}^{r^{\prime}}} \mathcal{R}_{L}^{r^{\prime}} \stackrel{\sim}{\rightarrow} \mathcal{R}_{L}^{r},
$$

which gives a Cartesian diagram of rigid spaces

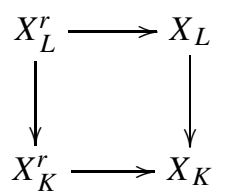

where $X_{K}=\bigcup_{r>0} X_{K}^{r}$ and $X_{L}=\bigcup_{r>0} X_{L}^{r}$. We put

$$
\tilde{\mathbb{f}}(T)=T^{p^{n}}+\left(\widetilde{a}_{p^{n}-1} \otimes 1\right) T^{p^{n}-1}+\cdots+\left(\widetilde{a}_{0} \otimes 1\right) \in\left(\mathcal{A}_{K} \widehat{\widehat{\otimes}}_{\mathcal{O}_{K_{0}}} \mathcal{O}_{K}\right)[T] .
$$

From (8.5.1) and (8.10.2), we have a surjection

$$
\tau_{L}: \mathcal{R}_{K}^{r}\langle T\rangle \rightarrow \mathcal{R}_{L}^{r}, \quad T \mapsto \tilde{h} \otimes 1,
$$

which induces an isomorphism that we denote abusively also by

$$
\tau_{L}: \mathcal{R}_{K}^{r}\langle T\rangle / \tilde{\mathbb{f}}(T) \stackrel{\sim}{\rightarrow} \mathcal{R}_{L}^{r} .
$$

In other terms, we have a co-Cartesian diagram of homomorphisms of $\mathcal{R}_{K}^{r}$-algebras

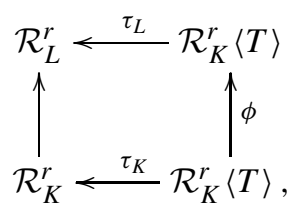

where $\phi(T)=\tilde{\mathbb{f}}(T)$ and $\tau_{K}(T)=0$. Hence, taking the union of the $K$-affinoid varieties associated to each of the $K$-affinoid algebras in (8.10.5) for $r \in \mathbb{Q} \geqslant 0$, we obtain a Cartesian diagram

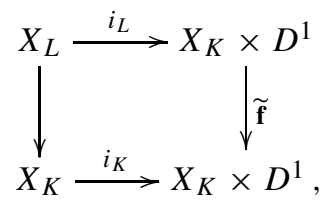


where $i_{L}, \tilde{\mathbf{f}}$ and $i_{K}$ are the morphisms induced by $\tau_{L}, \phi$ and $\tau_{K}$.

8.11. In the following, for any $0 \leqslant i \leqslant p^{n}-1$, we denote by $\alpha_{i}$ the element $a_{i}-\widetilde{a}_{i} \otimes 1 \in$ $I_{K}$ and fix $\tilde{\pi} \in \mathcal{A}_{K}$ a lifting of $\pi \in \mathcal{O}_{K}$. When the conductor $c>2$, for each $1 \leqslant i \leqslant p^{n}-1$, $v\left(a_{i}\right) \geqslant 2$ (Lemma 5.6). Let $a_{i}^{\prime}=\pi^{-2} a_{i} \in \mathcal{O}_{K}$ and $\widetilde{a}_{i}^{\prime} \in \mathcal{A}_{K}$ a lifting of $a_{i}^{\prime}$. Then we have $\tilde{a}_{i}=\tilde{\pi}^{2} \widetilde{a}_{i}^{\prime}+\tilde{y}_{i}$, where $\tilde{y}_{i} \in \operatorname{ker}\left(\mathcal{A}_{K} \rightarrow \mathcal{O}_{K}\right)$. We denote by $\alpha_{i}^{\prime}$ the element $a_{i}^{\prime}-\tilde{a}_{i}^{\prime} \otimes 1 \in I_{K}$ and by $\beta$ the element $\pi-\tilde{\pi} \otimes 1 \in I_{K}$. Then, we have

$$
\alpha_{i}=\left(a_{i}^{\prime}-\alpha_{i}^{\prime}\right)\left(2 \pi \beta-\beta^{2}\right)+\pi^{2} \alpha_{i}^{\prime}+\tilde{y}_{i} \otimes 1 .
$$

Since $\alpha_{i}^{\prime}, \beta \in I_{K} \subset \pi^{c} \mathcal{R}_{K, \mathcal{O}_{K}}^{c}$ and $\tilde{y}_{i} \otimes 1 \in I_{K}^{2}+\pi I_{K} \subset \pi^{c+1} \mathcal{R}_{K, \mathcal{O}_{K}}^{c}$ (Remark 8.8), we have $\alpha_{i} \in \pi^{c+1} \mathcal{R}_{K, \mathcal{O}_{K}}^{c}$. When $c=2$, we have $p=2, \sharp G=2$, deg $f=2$ and $\rho(c)=1$. Let $\widetilde{a}_{1}^{\prime \prime} \in \mathcal{A}_{K}$ be a lifting of $a_{1}^{\prime \prime}=\pi^{-1} a_{1}$. We have $\alpha_{1}=\tilde{\pi} \widetilde{a}_{1}^{\prime \prime}+\widetilde{z}_{1}$, where $z_{1} \in \operatorname{ker}\left(\mathcal{A}_{K} \rightarrow \mathcal{O}_{K}\right)$. We denote by $\alpha_{1}^{\prime \prime}$ the element $a_{1}^{\prime \prime}-\widetilde{a}_{1}^{\prime \prime} \otimes 1 \in I_{K}$. Then we have

$$
\alpha_{1}=\left(a_{1}^{\prime \prime}-\alpha_{1}^{\prime \prime}\right) \beta+\pi \alpha_{1}^{\prime \prime}+\widetilde{z}_{1} \otimes 1 .
$$

Since $\alpha_{1}^{\prime \prime}, \beta \in \pi^{c} \mathcal{R}_{K, \mathcal{O}_{K}}^{c}$ and $\widetilde{z}_{1} \otimes 1 \in I_{K}^{2}+\pi I_{K} \subset \pi^{c+1} \mathcal{R}_{K, \mathcal{O}_{K}}^{c}$, we have $\alpha_{1} \in \pi^{c} \mathcal{R}_{K, \mathcal{O}_{K}}^{c}$, and $\overline{\alpha_{1} / \pi^{c}}=\overline{a_{1}^{\prime \prime} \beta / \pi^{c}} \in \mathcal{R}_{K, \mathcal{O}_{K}}^{c} / \pi \mathcal{R}_{K, \mathcal{O}_{K}}^{c}$.

Put

$$
\widetilde{\mathbb{I}}_{0}(T)=\sum_{0 \leqslant i \leqslant p^{n}-1}\left(\alpha_{i} / \pi^{c}\right) \cdot T^{i} \in \mathcal{R}_{K, \mathcal{O}_{K}}^{c}[T]
$$

We have

$$
\tilde{\mathbb{f}}(T)=f(T)-\sum_{0 \leqslant i \leqslant p^{n}-1} \alpha_{i} T^{i}=f(T)-\pi^{c} \widetilde{\mathbb{f}}_{0}(T) .
$$

In the following, we fix an embedding $L \rightarrow \bar{K}$. Recall that we put $\sharp\left(G^{c}\right)=p^{s}$ (Subsection 5.1).

Proposition 8.12. The $K$-affinoid $X_{L}^{c}$ has $\sharp\left(G / G^{c}\right)=p^{n-s}$ geometric connected components. Let $\sigma_{1}, \ldots, \sigma_{p^{n-s}}$ be liftings of all the elements of $G / G^{c}$ in $G$. We have

$$
i_{L}\left(X_{L}^{c}\right) \subseteq \coprod_{1 \leqslant j \leqslant p^{n-s}} X_{K}^{c} \times\left(\sigma_{j}(h)+D^{1,(\rho(c))}\right) \subseteq X_{K} \times D^{1}
$$

PROOF. The proof is the same as in the equal characteristic case (Proposition 7.8).

In the following, we denote by $\overline{\mathfrak{X}}_{L, 0}^{c}$ the connected component of $\overline{\mathfrak{X}}_{L}^{c}$ corresponding to the connected component $X_{L, 0}^{c}$ of $X_{L}^{c}$ containing $(0, \ldots, 0, h) \in X_{K}^{c} \times D^{1}$ defined over $L$.

Proposition 8.13. There exists a canonical Cartesian diagram

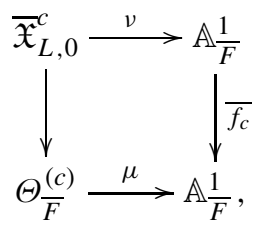


where $\overline{f_{c}}$ is defined in (5.4.1) and if $\xi$ is the canonical coordinate of $\mathbb{A} \frac{1}{F}$, we have

$$
\mu^{*}(\xi)= \begin{cases}\mathrm{d} a_{0} \otimes \pi^{-c}, & \text { if } c>2, \\ \left(a_{1}^{\prime \prime} h \mathrm{~d} \pi+\mathrm{d} a_{0}\right) \otimes \pi^{-2}, & \text { if } c=2 .\end{cases}
$$

Moreover, for any $\sigma \in G^{c}$, the following diagram

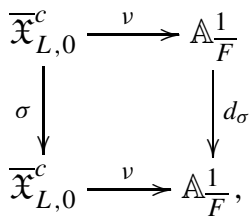

where $d_{\sigma}^{*}(\xi)=\xi-u_{\sigma}$ (Subsection 5.3), is commutative.

PROOF. We consider the $K$-affinoid algebra $\mathcal{R}_{K}^{c}$ (resp. $\mathcal{R}_{L}^{c}$ ) as a sub-ring of the $L$ affinoid algebra $\mathcal{R}_{K}^{c} \otimes_{K} L$ (resp. $\mathcal{R}_{L}^{c} \otimes_{K} L$ ). By (8.12), we have

$$
X_{L, 0}^{c}=i_{L}^{-1}\left(X_{K}^{c} \times\left(h+D^{1,(\rho(c))}\right)\right) \cap X_{L}^{c} .
$$

Hence $X_{L, 0}^{c}$ is presented by the $L$-affinoid algebra

$$
\left(\mathcal{R}_{L}^{c} \otimes_{K} L\right)\left\langle T^{\prime}\right\rangle /\left(\pi^{\rho(c)} T^{\prime}+h-\widetilde{h} \otimes 1\right) .
$$

By the isomorphism (8.10.4), (8.13.3) is isomorphic to

$$
\left(\mathcal{R}_{K}^{c} \otimes_{K} L\right)\left\langle T, T^{\prime}\right\rangle /\left(\widetilde{\mathbb{H}}(T), \pi^{\rho(c)} T^{\prime}+h-T\right),
$$

which, after eliminating $T$ by the relation $\pi^{\rho(c)} T^{\prime}+h-T=0$, is

$$
\left(\mathcal{R}_{K}^{c} \otimes_{K} L\right)\left\langle T^{\prime}\right\rangle /\left(\tilde{\mathbb{f}}\left(\pi^{\rho(c)} T^{\prime}+h\right)\right) .
$$

In both cases, by Proposition 5.4 and Subsection 8.11, we have

$$
\begin{gathered}
\tilde{\mathbb{H}}\left(\pi^{\rho(c)} T^{\prime}+h\right) / \pi^{c} \in \mathcal{R}_{K, \mathcal{O}_{L}}^{c}\left\langle T^{\prime}\right\rangle, \\
\widetilde{\mathbb{H}}\left(\pi^{\rho(c)} T^{\prime}+h\right) / \pi^{c+1} \notin \mathcal{R}_{K, \mathcal{O}_{L}}^{c}\left\langle T^{\prime}\right\rangle .
\end{gathered}
$$

Then the image of $\mathcal{R}_{K, \mathcal{O}_{L}}^{c}\left\langle T^{\prime}\right\rangle$ in (8.13.4) through the canonical surjective map

$$
\left(\mathcal{R}_{K}^{c} \otimes_{K} L\right)\left\langle T^{\prime}\right\rangle \rightarrow\left(\mathcal{R}_{K}^{c} \otimes_{K} L\right)\left\langle T^{\prime}\right\rangle /\left(\widetilde{\mathbb{H}}\left(\pi^{\rho(c)} T^{\prime}+h\right)\right),
$$

is

$$
\mathcal{R}_{K, \mathcal{O}_{L}}^{c}\left\langle T^{\prime}\right\rangle /\left(\widetilde{\mathbb{H}}\left(\pi^{\rho(c)} T^{\prime}+h\right) / \pi^{c}\right) .
$$

Extending scalars from $\mathcal{O}_{L}$ to $\bar{F}$, we obtain the following $\bar{F}$-algebra:

(i) If $c>2$,

$$
\left(\mathcal{R}_{K, \mathcal{O}_{L}}^{c} \otimes_{\mathcal{O}_{L}} \bar{F}\right)\left[T^{\prime}\right] /\left(\overline{f_{c}}\left(T^{\prime}\right)-\overline{\alpha_{0} / \pi^{c}}\right) .
$$

(ii) If $c=2$,

$$
\left(\mathcal{R}_{K, \mathcal{O}_{L}}^{c} \otimes_{\mathcal{O}_{L}} \bar{F}\right)\left[T^{\prime}\right] /\left(\overline{f_{2}}\left(T^{\prime}\right)-\overline{\left(\alpha_{0}+a_{1}^{\prime \prime} h \beta\right) / \pi^{2}}\right) .
$$


From the isomorphism (8.7.4) and the canonical exact sequence (4.10.3), we know that when $c>2$ (resp. $c=2), \overline{\alpha_{0} / \pi^{c}}\left(\right.$ resp. $\overline{\left(\alpha_{0}+a_{1}^{\prime \prime} h \beta\right) / \pi^{2}}$ ) is a non-zero linear term in $\mathcal{R}_{K, \mathcal{O}_{L}}^{c} \otimes_{\mathcal{O}_{L}}$ $\bar{F}$. Hence (8.13.6) and (8.13.7) are all reduced. Then, by ([AS1, 4.1]),

$$
\operatorname{Spf}\left(\mathcal{R}_{K, \mathcal{O}_{L}}^{c}\left\langle T^{\prime}\right\rangle /\left(\widetilde{\mathbb{f}}\left(\pi^{\rho(c)} T^{\prime}+h\right) / \pi^{c}\right)\right)
$$

is the normalized integral model of $X_{K, 0}^{c}$ defined over $\mathcal{O}_{L}$. Hence $\overline{\mathfrak{X}}_{L, 0}^{c}$ is defined by the $\bar{F}$-algebra (8.13.6) (resp. (8.13.7)) when $c>2$ (resp. $c=2)$. We put

$$
v: \overline{\mathfrak{X}}_{L, 0}^{c} \rightarrow \mathbb{A} \frac{1}{F}=\operatorname{Spec}(\bar{F}[\xi]), \quad v^{*}(\xi)=T^{\prime} .
$$

It follows form the isomorphism $\overline{\mathfrak{X}}_{K}^{c} \rightarrow \Theta_{\bar{F}}^{(c)}$ that (8.13.1) is Cartesian.

For any $\sigma \in G^{c}$, let $y_{\sigma}(x)=b_{r} x^{r}+\cdots+b_{0} \in \mathcal{O}_{K}[x]$ be a polynomial such that $y_{\sigma}(h)=(h-\sigma(h)) / \pi^{\rho(c)} \in \mathcal{O}_{L}$. We denote by $\tilde{y}_{\sigma}(x)=\widetilde{b}_{r} x^{r}+\cdots+\widetilde{b}_{0}$ a lifting of $y_{\sigma}(x)$ in $\mathcal{A}_{K}[x]$ and by $\widetilde{y}(x)$ the polynomial

$$
\widetilde{\mathbb{Y}}(x)=\left(\tilde{b}_{r} \otimes 1\right) x^{r}+\cdots+\left(\tilde{b}_{0} \otimes 1\right) \in\left(\mathcal{A}_{K} \widehat{\widehat{\otimes}}_{\mathcal{O}_{K_{0}}} \mathcal{O}_{K}\right)[x] .
$$

Let $\mathrm{g}_{\sigma}: \mathcal{A}_{L} \rightarrow \mathcal{A}_{L}$ be a homomorphism as in (8.9.1). We denote by $\mathbf{g}_{\sigma}$ the induced morphism of $g_{\sigma}$ on (8.13.5). By (8.10.1), we have

$$
\operatorname{ker}\left(\mathcal{A}_{L} \rightarrow \mathcal{O}_{L}\right)=\bigoplus_{i=0}^{p^{n}-1} \operatorname{ker}\left(\mathcal{A}_{K} \rightarrow \mathcal{O}_{K}\right) \tilde{h}^{i}
$$

Hence, we have $g_{\sigma}(\widetilde{h})=\tilde{h}-\tilde{\pi}^{\rho(c)} \tilde{y}_{\sigma}(\widetilde{h})+\varepsilon(\widetilde{h})$, where $\varepsilon$ is a polynomial with coefficients in $\operatorname{ker}\left(\mathcal{A}_{K} \rightarrow \mathcal{O}_{K}\right)$. Then, we have

$$
\mathbf{g}_{\sigma}\left(T^{\prime}\right)=T^{\prime}-\widetilde{\mathbb{Y}}_{\sigma}\left(\pi^{\rho(c)} T^{\prime}+h\right)+\Delta\left(T^{\prime}\right),
$$

where

$$
\Delta\left(T^{\prime}\right)=-\left(\left(\tilde{\pi}^{\rho(c)} \otimes 1-\pi^{\rho(c)}\right) / \pi^{\rho(c)}\right) \widetilde{\mathbb{Y}}_{\sigma}\left(\pi^{\rho(c)} T^{\prime}+h\right)+\widetilde{\varepsilon}\left(\pi^{\rho(c)} T^{\prime}+h\right) / \pi^{\rho(c)},
$$

and $\widetilde{\varepsilon}$ is a polynomials with coefficients in $J=\left\{\widetilde{x} \otimes 1 \in \mathcal{A} \widehat{\widehat{\otimes}} \mathcal{O}_{K_{0}} \mathcal{O}_{K} ; \tilde{x} \in \operatorname{ker}\left(\mathcal{A}_{K} \rightarrow \mathcal{O}_{K}\right)\right\}$. Since $J \subseteq \pi^{c+1} \mathcal{R}_{K, \mathcal{O}_{K}}$ (Remark 8.8), $\tilde{\pi}^{\rho(c)} \otimes 1-\pi^{\rho(c)} \in \pi^{c} \mathcal{R}_{K, \mathcal{O}_{K}}$ and $c>\rho(c)$, it is easy to see that the reduction of $\Delta\left(T^{\prime}\right)$ to $\overline{\mathfrak{X}}_{L, 0}^{c}$ is zero. For any $0 \leqslant j \leqslant r$, we have $\widetilde{b}_{j} \otimes 1-b_{j} \in \pi^{c} \mathcal{R}_{K, \mathcal{O}_{K}}^{c}$. Then

$$
\overline{\widetilde{\mathbb{y}}_{\sigma}\left(\pi^{\rho(c)} T^{\prime}+h\right)}=\overline{\widetilde{y}_{\sigma}\left(\pi^{\rho(c)} T^{\prime}+h\right)}=\overline{\widetilde{y}_{\sigma}(h)}=u_{\sigma} .
$$

Hence, by ([AS2, 2.13]), the diagram (8.13.2) is commutative. 


\section{The refined Swan conductor of an extension of type (II).}

9.1. In this section, we assume either that $K$ has characteristic $p$ or that it has characteristic 0 and that $p$ is not a uniformizer of $K$. Let $L$ be a finitely generated extension of $K$ of type (II) and we take again the notation and assumptions of Subsections 5.1, 5.2, 7.9 and 8.13 .

PROPOSITION 9.2. The fibre product $\overline{\mathfrak{X}}_{L, 0}^{c} \times_{\Theta} \frac{(c)}{\bar{F}} \Xi_{\bar{F}}^{(c)}$ (4.10.4) is a connected affine scheme.

Proof. The image of $\mathrm{d} a_{0} \otimes 1$ and $\left(a_{1}^{\prime \prime} h \mathrm{~d} \pi+\mathrm{d} a_{0}\right) \otimes 1$ by the canonical map from $\widehat{\Omega}_{\mathcal{O}_{K} / F_{0}}^{1} \otimes_{\mathcal{O}_{K}} \bar{F}$ (resp. $\widehat{\Omega}_{\mathcal{O}_{K} / \mathcal{O}_{K_{0}}}^{1} \otimes_{\mathcal{O}_{K}} \bar{F}$ ) to $\Omega_{F}^{1} \otimes_{F} \bar{F}$ is $\mathrm{d} \bar{a}_{0} \otimes 1$, which is a non-zero element. So we have a Cartesian diagram

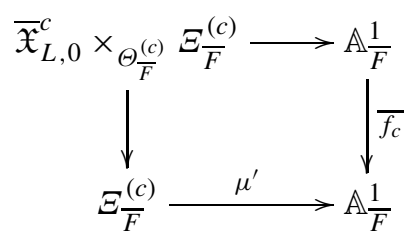

where $\mu^{\prime *}(\xi)=\mathrm{d} \bar{a}_{0} \otimes \pi^{-c}$. Since $\mathrm{d} \bar{a}_{0} \otimes \pi^{-c}$ is a non-zero linear term in the affine space $\Xi_{\bar{F}}^{(c)}, \overline{\mathfrak{X}}_{L, 0}^{c} \times_{\Theta \frac{(c)}{F}} \Xi_{\bar{F}}^{(c)}$ is connected.

9.3. Proof of Proposition 5.7. By ([AS2, 5.13]), both in the equal and unequal characteristic case, we have a commutative diagram

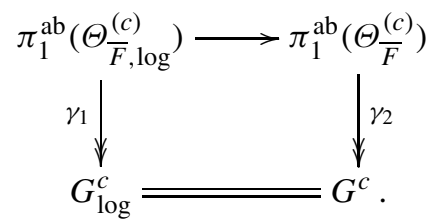

The surjection $\gamma_{1}$ factors through $\pi_{1}^{\mathrm{alg}}\left(\Theta_{\bar{F}, \log }^{(c)}\right)$ (Theorem 4.11). By Propositions 7.9 and 8.13, $\gamma_{2}$ also factors through $\pi_{1}^{\mathrm{alg}}\left(\Theta_{\bar{F}}^{(c)}\right)$. Combining (9.3.1) and the following canonical commutative diagram

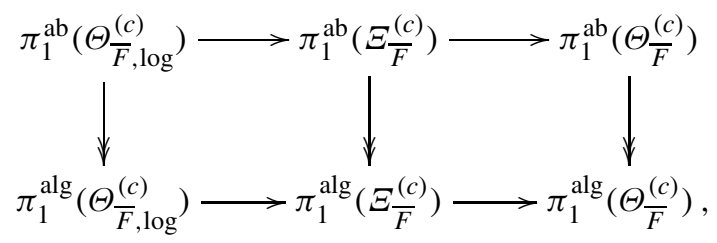


we obtain that

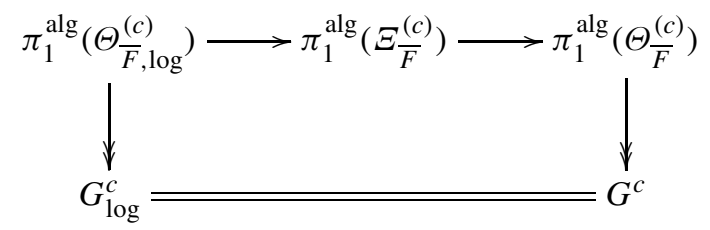

is commutative. The composition of morphisms $\pi_{1}^{\mathrm{alg}}\left(\Xi_{\frac{\mathrm{F}}{F}}^{(c)}\right) \rightarrow \pi_{1}^{\mathrm{alg}}\left(\Theta_{\frac{(c)}{F}}^{(c)} \rightarrow G^{c}\right.$ corresponds to the isogeny $\overline{\mathfrak{X}}_{L, 0}^{c} \times_{\Theta \frac{(c)}{F}} \Xi_{\bar{F}}^{(c)} \rightarrow \Xi_{\bar{F}}^{(c)}$ (cf. (9.2.1)). Hence, by (9.3.2), we have a commutative diagram

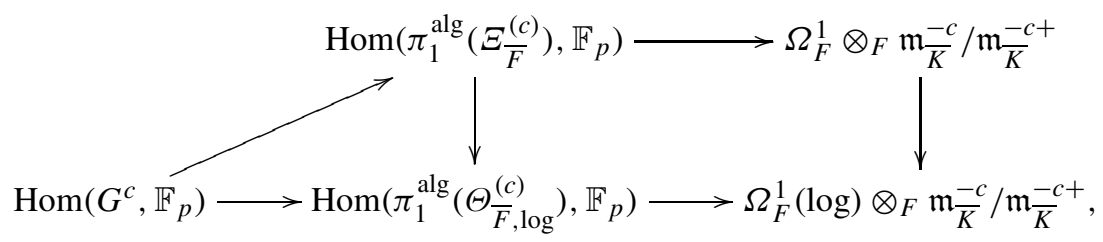

which concludes Proposition 5.7.

9.4. Proof of Theorem 5.9. Since the surjection $\pi_{1}^{\text {alg }}\left(\Xi_{\bar{F}}^{(c)}\right) \rightarrow G^{c}$ is obtained by pulling-back the isogeny $\overline{f_{c}}: \mathbb{A} \frac{1}{F} \rightarrow \mathbb{A} \frac{1}{F}$ by $\mu^{\prime}$ (cf. (9.2.1)), it is an étale $G^{c}$-torsor with the action of $G^{c}$ given by $d_{\sigma}$ for $\sigma \in G^{c}$ (7.9.2), (8.13.2). With notation in Subsection 5.8, we denote by $\tilde{f}_{c, \chi}(\xi)$ the polynomial

$$
\tilde{f}_{c, \chi}(\xi)=\left(\prod_{\sigma \in G-G^{c}} u_{\sigma}\right)\left(\xi^{p}-\bar{f}_{c, \chi}^{p-1}\left(u_{\tau}\right) \xi\right) \in \bar{F}[\xi] .
$$

Observe that $\tilde{f}_{c, \chi}\left(\bar{f}_{c, \chi}(\xi)\right)=\overline{f_{c}}(\xi)$, hence the isogeny $\overline{f_{c}}$ is the composition of two isogenies

$$
\mathbb{A} \frac{1}{F} \stackrel{\bar{f}_{c, x}}{\longrightarrow} \mathbb{A} \frac{1}{F} \stackrel{\tilde{f}_{c, x}}{\longrightarrow} \mathbb{A} \frac{1}{F}
$$

For any $\sigma \in \operatorname{ker} \chi, \bar{f}_{c, \chi}^{*}\left(\xi-u_{\sigma}\right)=\bar{f}_{c, \chi}^{*}(\xi)$, i.e. $\bar{f}_{c, \chi} d_{\sigma}=\bar{f}_{c, \chi}$. Hence the isogeny $\tilde{f}_{c, \chi}$ : $\mathbb{A} \frac{1}{F} \rightarrow \mathbb{A} \frac{1}{F}$ is an étale $\left(G^{c} / \operatorname{ker} \chi\right)$-torsor. Then, the surjection $\pi_{1}^{\text {alg }}\left(\Xi_{\bar{F}}^{(c)}\right) \rightarrow G^{c} \stackrel{\chi}{\rightarrow} \mathbb{F}_{p}$ corresponds to the pull-back of $\tilde{f}_{c, \chi}$ by $\mu^{\prime}$ and the $\mathbb{F}_{p}$-action on this torsor is given by $1^{*}$ : $\xi \mapsto \xi-\bar{f}_{c, \chi}\left(u_{\tau}\right)$. We have the following Cartesian diagram

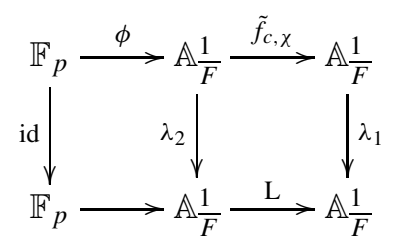


where $\mathrm{L}$ denotes the Lang's isogeny defined by $\mathrm{L}^{*}(\xi)=\xi^{p}-\xi$. The morphisms $\lambda_{1}, \lambda_{2}$ and $\phi$ are given as follows

$$
\begin{gathered}
\lambda_{1}^{*}(\xi)=-\xi /\left(\prod_{\sigma \in G-G^{c}} u_{\sigma}\right) \bar{f}_{c, \chi}^{p}\left(u_{\tau}\right), \\
\lambda_{2}^{*}(\xi)=-\xi / \bar{f}_{c, \chi}\left(u_{\tau}\right), \\
\phi(1)=-\bar{f}_{c, \chi}\left(u_{\tau}\right) .
\end{gathered}
$$

The sign is chosen in order that, for any $\sigma \in G^{c}$, the translation by $\phi(\chi(\sigma))$ is induced by $d_{\sigma}$. Consequently, $\pi_{1}^{\text {alg }}\left(\Xi_{\bar{F}}^{(c)}\right) \rightarrow G^{c} \stackrel{\chi}{\rightarrow} \mathbb{F}_{p}$ corresponds to the pull-back of $\mathrm{L}$ by $\lambda_{1} \mu^{\prime}$. Hence the image of $\chi \in \operatorname{Hom}\left(G^{c}, \mathbb{F}_{p}\right)$ in $\Omega_{F}^{1} \otimes_{F} \mathfrak{m}_{\bar{K}}^{-c} / \mathfrak{m}_{\bar{K}}^{-c+}(9.3 .3)$ is

$$
-\mathrm{d} \bar{a}_{0} \otimes \frac{\pi^{-c}}{\left(\prod_{\sigma \in G-G^{c}} u_{\sigma}\right) \bar{f}_{c, \chi}^{p}\left(u_{\tau}\right)} \in \Omega_{F}^{1} \otimes_{F} \mathfrak{m}_{K}^{-c} / \mathfrak{m}_{\bar{K}}^{-c+} .
$$

Then the theorem follows from (9.3.3).

\section{Comparison of Kato's and Abbes-Saito's characteristic cycles.}

10.1. In this section, let $L$ be a finite Galois extension of $K$ of type (II) and we take again the notation and assumptions of Subsections 5.1 and 5.2. Let $C$ be an algebraically closed field of characteristic zero. We fix a non-trivial character $\psi_{0}: \mathbb{F}_{p} \rightarrow C^{\times}$. Any character $\chi: G^{c} \rightarrow C^{\times}$factors uniquely through $G^{c} \rightarrow \mathbb{F}_{p} \stackrel{\psi_{0}}{\rightarrow} C^{\times}$. We denote by $\bar{\chi}$ the induced character $G^{c} \rightarrow \mathbb{F}_{p}$.

PROPOSITION 10.2. Let $\chi: G \rightarrow C^{\times}$be a character of $G$ such that its restriction to $G^{c}$ is non-trivial. Let $\tau \in G^{c}$ be a lifting of $1 \in \mathbb{F}_{p}$ in $G^{c}$ through $\bar{\chi}: G^{c} \rightarrow \mathbb{F}_{p}$. Then Kato's Swan conductor with differential values $\operatorname{sw}_{\psi_{0}(1)}(\chi)$ is given by

$$
\operatorname{sw}_{\psi_{0}(1)}(\chi)=\left[\pi^{c}\right]+\left[-\bar{f}_{c, \bar{\chi}}^{p}\left(u_{\tau}\right)\right]+\sum_{\sigma \in G-G^{c}}\left[u_{\sigma}\right]-\left[\mathrm{d} \bar{a}_{0}\right] \in S_{K, L}
$$

(Subsection 5.3, Subsection 5.8).

Proof. By definition (3.13.1), we have

$$
\begin{aligned}
\operatorname{sw}_{\psi_{0}(1)}(\chi)= & \sum_{\sigma \in G-\{1\}}([h-\sigma(h)]-[\mathrm{d} \bar{h}]) \otimes(1-\chi(\sigma))+\sum_{r \in \mathbb{F}_{p}^{\times}}[r] \otimes \psi_{0}(r) \\
= & \sum_{\sigma \in G^{c}-\{1\}}[h-\sigma(h)] \otimes(1-\chi(\sigma))+\sum_{r \in \mathbb{F}_{p}^{\times}}[r] \otimes \psi_{0}(r) \\
& +\sum_{\sigma \in G-G^{c}}[h-\sigma(h)]-\sum_{\sigma \in G-G^{c}}[h-\sigma(h)] \otimes \chi(\sigma) \\
& -\sum_{\sigma \in G-\{1\}}[\mathrm{d} \bar{h}] \otimes(1-\chi(\sigma)) .
\end{aligned}
$$


Choose an $\mathbb{F}_{p}$-basis $\tau_{1}=\tau, \tau_{2}, \ldots, \tau_{s}$ of $G^{c}$ such that $\bar{\chi}\left(\tau_{1}\right)=1 \in \mathbb{F}_{p}$ and that for any $2 \leqslant j \leqslant s, \bar{\chi}\left(\tau_{j}\right)=0$. Then, by Lemma 5.5, we have

$$
\text { (10.2.2) } \begin{aligned}
& \sum_{\sigma \in G^{c}-\{1\}}[h-\sigma(h)] \otimes(1-\chi(\sigma))+\sum_{r \in \mathbb{F}_{p}^{\times}}[r] \otimes \psi_{0}(r) \\
= & {\left[\pi^{\rho(c) \sharp G^{c}}\right]+\sum_{\left\{j_{1}, \ldots, j_{s}\right\} \in \mathbb{F}_{p}^{s}-\{0\}}\left[j_{1} u_{\tau_{1}}+\cdots+j_{s} u_{\tau_{s}}\right] \otimes\left(1-\psi_{0}\left(j_{1}\right)\right)+\sum_{r \in \mathbb{F}_{p}^{\times}}[r] \otimes \psi_{0}(r) } \\
= & {\left[\pi^{\rho(c) \sharp G^{c}}\right]+\sum_{r \in \mathbb{F}_{p}^{\times}}\left[\bar{f}_{c, \bar{\chi}}\left(r u_{\tau_{1}}\right)\right] \otimes\left(1-\psi_{0}(r)\right)+\sum_{r \in \mathbb{F}_{p}^{\times}}[r] \otimes \psi_{0}(r) } \\
= & {\left[\pi^{\rho(c) \sharp G^{c}}\right]+\sum_{r \in \mathbb{F}_{p}^{\times}}\left(\left[\bar{f}_{c, \bar{\chi}}\left(u_{\tau_{1}}\right)\right]+[r]\right) \otimes\left(1-\psi_{0}(r)\right)+\sum_{r \in \mathbb{F}_{p}^{\times}}[r] \otimes \psi_{0}(r) } \\
= & {\left[\pi^{\rho(c) \sharp G^{c}}\right]+\sum_{r \in \mathbb{F}_{p}^{\times}}\left[\bar{f}_{c, \bar{\chi}}\left(u_{\tau_{1}}\right)\right] \otimes\left(1-\psi_{0}(r)\right)+\sum_{r \in \mathbb{F}_{p}^{\times}}[r] } \\
= & {\left[\pi^{\rho(c) \sharp G^{c}}\right]+\left[-\bar{f}_{c, \bar{\chi}}^{p}\left(u_{\tau_{1}}\right)\right] \in S_{L / K} . }
\end{aligned}
$$

Let $\sigma_{1}=1, \sigma_{2}, \ldots, \sigma_{p^{n-s}}$ be liftings of all the elements of $G / G^{c}$ in $G$ and denote by $J$ the set $\left\{\sigma_{2}, \ldots, \sigma_{p^{n-s}}\right\}$. Observe that for any $\varsigma \in J$ and $\sigma \in G^{c}$, we have

$$
[h-\varsigma \sigma(h)]=[h-\varsigma(h)+\varsigma(h-\sigma(h))]=[h-\varsigma(h)] .
$$

Hence

$$
\begin{aligned}
\sum_{\sigma \in G-G^{c}}[h-\sigma(h)] \otimes \chi(\sigma) & =\sum_{\varsigma \in J} \sum_{\sigma \in G^{c}}[h-\varsigma \sigma(h)] \otimes \chi(\varsigma \sigma) \\
& =\sum_{\varsigma \in J} \sum_{\sigma \in G^{c}}[h-\varsigma(h)] \otimes \chi(\varsigma) \chi(\sigma)=0 .
\end{aligned}
$$

Moreover, by the isomorphism (3.4.1), we have

$$
\sum_{\sigma \in G^{c}-\{1\}}[\mathrm{d} \bar{h}] \otimes(1-\chi(\sigma))=\sharp G[\mathrm{~d} \bar{h}]=\left[\mathrm{d} \bar{a}_{0}\right] \in S_{K, L} .
$$

Hence, combining (10.2.1), (10.2.2), (10.2.3) and (10.2.4), we obtain that

$$
\begin{aligned}
\operatorname{sW}_{\psi_{0}(1)}(\chi) & =\left[\pi^{\rho(c) \sharp G^{c}}\right]+\left[-\bar{f}_{c, \chi}^{p}\left(u_{\tau_{1}}\right)\right]+\sum_{\sigma \in G-G^{c}}[h-\sigma(h)] \otimes 1-\sharp G[\mathrm{~d} \bar{h}] \\
& =\left[\pi^{c}\right]+\left[-\bar{f}_{c, \bar{\chi}}^{p}\left(u_{\tau}\right)\right]+\sum_{\sigma \in G-G^{c}}\left[u_{\sigma}\right]-\left[\mathrm{d} \bar{a}_{0}\right] .
\end{aligned}
$$

LEMMA 10.3. Let $M$ be a finite dimensional $C$-vector space with an irreducible linear action of $G$. Then, there exist a subgroup $H$ of $G$ satisfying $G^{c} \subseteq H$ and a 1-dimensional representation $\theta$ of $H$, such that $M=\operatorname{Ind}_{H}^{G} \theta$. 
Proof. Since $M$ is irreducible and $G$ is nilpotent (hence super-solvable), there exist a subgroup $H$ of $G$ and a 1-dimensional representation $\theta$ of $H$, such that $M=\operatorname{Ind}_{H}^{G} \theta$ ([Se2, 8.5 Theorem 16]). Let $\operatorname{Res}_{G^{c}}^{G} M=\bigoplus_{i} M_{i}$ be the canonical decomposition of $\operatorname{Res}_{G^{c}}^{G} M$ into isotypic $G^{c}$-representations (cf. [Se2, 2.6]). Since $G^{c}$ is contained in the center of $G$, any $\sigma \in G$ defines an automorphism of the $G^{c}$-representation $\operatorname{Res}_{G^{c}}^{G} M$. In particular, for any $i$, $\sigma$ induces an automorphism of $M_{i}$. On the other hand, since $M$ is irreducible, $G$ permutes transitively the $M_{i}$ 's. Hence $\operatorname{Res}_{G^{c}}^{G} M$ is isotypic. By ([Se2, 7.3 Propsition 22]), we have

$$
\operatorname{Res}_{G^{c}}^{G} M=\operatorname{Res}_{G^{c}}^{G} \operatorname{Ind}_{H}^{G} \theta=\bigoplus_{H \backslash G / G^{c}} \operatorname{Ind}_{H \cap G^{c}}^{G^{c}} \operatorname{Res}_{H \cap G^{c}}^{H} \theta .
$$

We notice that, if $H \cap G^{c} \neq G^{c}$, since $G^{c}=H \cap G^{c} \oplus G^{c} / H \cap G^{c}, \operatorname{Ind}_{H \cap G^{c}}^{G^{c}} \operatorname{Res}_{H \cap G^{c}}^{H} \theta$ is isomorphic to the tensor of the regular representation of $G^{c} / H \cap G^{c}$ with $\operatorname{Res}_{H \cap G^{c}}^{H} \theta$ which is not isotypic.

THEOREM 10.4. Assume that $p$ is not a uniformizer of $K$. Let $M$ be a finite dimensional $C$-vector space with a linear action of $G$. Then,

$$
\mathrm{CC}_{\psi_{0}}(M)=\mathrm{KCC}_{\psi_{0}(1)}(M) .
$$

Proof. From the definitions, we may assume that $M$ is irreducible. We denote by $c_{0}$ the unique slope of $M$. By definitions and Proposition 3.14, both sides of (10.4.1) will not change if replacing $G$ by $G / G^{c_{0}+}$. Hence we may assume further that the unique slope of $M$ is equal to $c$. By Lemma $10.3, M=\operatorname{Ind}_{H}^{G} \theta$ where $H$ is a subgroup of $G$ containing $G^{c}$ and $\theta$ is a character of $H$. Since the slope of $M$ is $c$, the restriction of $\theta$ to $G^{c}$ is non-trivial (10.3.1). We notice that $[G: H]=\operatorname{dim}_{C} M$. Choose an $\mathbb{F}_{p}$-basis $\tau_{1}, \ldots, \tau_{s}$ of $G^{c}$ such that $\bar{\theta}\left(\tau_{1}\right)=1 \in \mathbb{F}_{p}$ and, for any $2 \leqslant j \leqslant s, \bar{\theta}\left(\tau_{j}\right)=0$. Let $c^{\prime}=\rho(c)+\sum_{\sigma \in H-\{1\}} v(h-\sigma(h))$. Since $L / L^{H}$ is still of type (II), we obtain that the conductor of $L / L^{H}$ is $c^{\prime}$, that $H^{c^{\prime}}=G^{c}$ and, denoting by $\rho^{\prime}$ the Herbrand function of $L / L^{H}$, that $\rho^{\prime}\left(c^{\prime}\right)=\rho(c)$. Using Proposition 10.2 for the group $H$ and the representation $\theta$, we have

$$
\operatorname{sw}_{\psi_{0}(1)}(\theta)=\left[\pi^{c^{\prime}}\right]+\left[-\bar{f}_{c, \bar{\theta}}^{p}\left(u_{\tau_{1}}\right)\right]+\sum_{\sigma \in H-H^{c^{\prime}}}\left[u_{\sigma}\right]-\sharp H[\mathrm{~d} \bar{h}] .
$$

Meanwhile, we have

$$
-\sum_{\sigma \in G-H}([\mathrm{~d} \bar{h}]-[h-\sigma(h)])=(\sharp H-\sharp G)[\mathrm{d} \bar{h}]+\left[\pi^{c-c^{\prime}}\right]+\sum_{\sigma \in G-H} u_{\sigma} .
$$

Hence, combining (10.4.2), (10.4.3) and the induction formula for Kato's Swan conductors (3.15.2), we have

$$
\begin{aligned}
\operatorname{sw}_{\psi_{0}(1)}(M) & =[G: H]\left(\operatorname{sw}_{\psi_{0}(1)}(\theta)-\sum_{\sigma \in G-H}([\mathrm{~d} \bar{h}]-[h-\sigma(h)])\right. \\
& =[G: H]\left(\left[\pi^{c}\right]+\left[-\bar{f}_{c, \bar{\theta}}^{p}\left(u_{\tau_{1}}\right)\right]-\left[\mathrm{d} \bar{a}_{0}\right]+\sum_{\sigma \in G-G^{c}}\left[u_{\sigma}\right]\right) .
\end{aligned}
$$


Hence Kato's characteristic cycle $\mathrm{KCC}_{\psi_{0}(1)}(M)$ is given by

$$
\mathrm{KCC}_{\psi_{0}(1)}(M)=\frac{\left(-\mathrm{d} \bar{a}_{0}\right)^{\otimes[G: H]}}{\left(\left(\prod_{\sigma \in G-G^{c}} u_{\sigma}\right) \bar{f}_{c, \bar{\theta}}^{p}\left(u_{\tau_{1}}\right)\right)^{[G: H]}} \in\left(\Omega_{F}^{1}\right)^{\otimes[G: H]} .
$$

On the other hand, $\operatorname{Res}_{G^{c}}^{G} M=\bigoplus_{G / H} \operatorname{Res}_{G^{c}}^{H} \theta$ (10.3.1). Hence the Abbes-Saito's characteristic cycle $\mathrm{CC}_{\psi_{0}}(M)$ is given by

$$
\begin{aligned}
\mathrm{CC}_{\psi_{0}}(M) & =\left(\operatorname{rsw}\left(\operatorname{Res}_{G^{c}}^{H}(\theta)\right) \otimes \pi^{c}\right)^{[G: H]} \\
& =\frac{\left(-\mathrm{d} \bar{a}_{0}\right)^{\otimes[G: H]}}{\left(\left(\prod_{\sigma \in G-G^{c}} u_{\sigma}\right) \bar{f}_{c, \bar{\theta}}^{p}\left(u_{\tau_{1}}\right)\right)^{[G: H]}} \in\left(\Omega_{F}^{1} \otimes_{F} \bar{F}\right)^{\otimes[G: H]} .
\end{aligned}
$$

So, we have $\mathrm{CC}_{\psi_{0}}(M)=\mathrm{KCC}_{\psi_{0}(1)}(M)$.

COROLlary 10.5. Assume that $p$ is not a uniformizer of $K$. Let $M$ be a finite dimensional $\Lambda$-vector space with a linear action of $G$ and $r=\operatorname{dim}_{\Lambda} M / M^{(0)}$. Then, we have

$$
\mathrm{CC}_{\psi_{0}}(M) \in\left(\Omega_{F}^{1}\right)^{r} \subseteq\left(\Omega_{F}^{1} \otimes_{F} \bar{F}\right)^{r} .
$$

It is a Hasse-Arf type result for Abbes-Saito characteristic cycle. We should mention that T. Saito ([Sa4, 3.10]) and L. Xiao [Xiao] proved independently analogue results for smooth varieties of any dimension over perfect fields

COROLlary 10.6. Assume that $p$ is not a uniformizer of $K$. Let $H$ be a sub-group of $G$, and $N$ a finite dimensional $C$-linear representation of $H$. We denote by $r$ the dimension of $N$ and by $r^{\prime}$ the dimension of $N^{(0)}$. Then, we have

$$
\mathrm{CC}_{\psi_{0}}\left(\operatorname{Ind}_{H}^{G} N\right)=\mathrm{CC}_{\psi_{0}}(N)^{\otimes[G: H]} \otimes \frac{\left(\mathrm{d} \bar{a}_{0}\right)^{\otimes([G: H]-1)}}{\left(\prod_{\sigma \in G-H} u_{\sigma}\right)^{[G: H]}} \in\left(\Omega_{F}^{1}\right)^{\otimes\left([G: H] r-r^{\prime}\right)} .
$$

Indeed, (10.6.1) follows from the induction formula for Kato's Swan conductor with differential values (3.15.2) and Theorem 10.4.

REMARK 10.7. Assume that $p$ is not a uniformizer of $K$. Let $L^{\prime}$ be a finite Galois extension of $K$ of group $G^{\prime}$ which contains a sub-extension $K^{\prime}$ of $K$ such that $K^{\prime} / K$ is unramified and $L^{\prime} / K^{\prime}$ is of type (II). We denote by $P^{\prime}$ the Galois group of the extension $L^{\prime} / K^{\prime}$ and by $F^{\prime}$ the residue field of $\mathcal{O}_{K^{\prime}}$. Let $\Lambda$ be a finite field of characteristic $\ell \neq p$ which contains a primitive $\left(\sharp P^{\prime}\right)$-th root of unity and let $N$ be a $\Lambda$-vector space of finite dimension with a linear- $G^{\prime}$ action. We fix a non-trivial character $\psi: \mathbb{F}_{p} \rightarrow \Lambda^{\times}$. By Remarks 3.18 and 3.19, we can still define $\operatorname{KCC}_{\psi(1)}(N) \in\left(\Omega_{F}^{1}\right)^{\otimes r}$, where $r=\operatorname{dim}_{\Lambda} N / N^{(0)}$. On the other hand, the wild inertia subgroup $P$ of $G_{K}$ acts on $N$ through $P^{\prime}$, we can define $\mathrm{CC}_{\psi}(N)$ (Subsection 4.12). By [Sa2, 1.22] and [Sa3, 3.1], we have

$$
\mathrm{CC}_{\psi}\left(\operatorname{Res}_{P^{\prime}}^{G^{\prime}} N\right)=\mathrm{CC}_{\psi}(N) \in\left(\Omega_{F}^{1}(\log ) \otimes_{F} \bar{F}\right)^{\otimes r}
$$

through the canonical isomorphism $\Omega_{F}^{1}(\log ) \otimes_{F} F^{\prime} \stackrel{\sim}{\rightarrow} \Omega_{F^{\prime}}^{1}(\log )$. Moreover, let $\Lambda^{\prime}$ be the algebraic closure of the fraction field of the ring of Witt vectors $W(\Lambda), N^{\prime}$ a pre-image of the 
class of $\operatorname{Res}_{P^{\prime}}^{G^{\prime}} N$ in the Grothendieck ring $R_{\Lambda^{\prime}}\left(P^{\prime}\right)\left(\left[\operatorname{Se} 2,16.1\right.\right.$ Theorem 33] ) and $\psi^{\prime}: \mathbb{F}_{p} \rightarrow$ $\Lambda^{\prime \times}$ the unique lifting of $\psi: \mathbb{F}_{p} \rightarrow \Lambda^{\times}$. By Lemma 4.8, we deduce that

$$
\mathrm{CC}_{\psi^{\prime}}\left(N^{\prime}\right)=\mathrm{CC}_{\psi}\left(\operatorname{Res}_{P^{\prime}}^{G^{\prime}} N\right) \text {. }
$$

From Theorem 10.4, we have

$$
\mathrm{CC}_{\psi^{\prime}}\left(N^{\prime}\right)=\operatorname{KCC}_{\psi(1)}(N) .
$$

By (10.7.1), (10.7.2) and (10.7.3), we conclude that

$$
\mathrm{CC}_{\psi}(N)=\operatorname{KCC}_{\psi(1)}(N) \in\left(\Omega_{F}^{1}\right)^{\otimes r} .
$$

\section{Nearby cycles of $\ell$-sheaves on relative curves.}

11.1. In this section, we denote by $S=\operatorname{Spec}(R)$ an excellent strictly henselian trait. Assume that the residue field of $R$ has characteristic $p$ and that $p$ is not a uniformizer of $R$. We denote by $s$ (resp. $\eta$, resp. $\bar{\eta}$ ) the closed point (resp. generic point, a geometric generic point) of $S$. A finite covering of $(S, \eta, s)$ stands for a trait $\left(S^{\prime}, \eta^{\prime}, s^{\prime}\right)$ equipped with a finite morphism $S^{\prime} \rightarrow S$. Let $\Lambda$ be a finite field of characteristic $\ell \neq p$ and fix a non-trivial character $\psi_{0}: \mathbb{F}_{p} \rightarrow \Lambda^{\times}$.

11.2. We define a category $\mathscr{C}_{S}$ as follows. An object of $\mathscr{C}_{S}$ is a normal affine $S$-scheme $H$ for which there exist a flat $S$-scheme of relative dimension one $X$ and a closed point $x$ of $X_{s}$, such that $X-\{x\}$ is smooth over $S$ and $H$ is $S$-isomorphic to the henselization of $X$ at $x$. A morphism between two objects of $\mathscr{C}_{S}$ is a generically étale finite morphism of $S$-schemes. Let $\left(S^{\prime}, \eta^{\prime}, s^{\prime}\right)$ be a finite covering of $(S, \eta, s)$. Then for any object $H$ of $\mathscr{C}_{S}, H \times{ }_{S} S^{\prime}$ is an object of $\mathscr{C}_{S^{\prime}}([$ Kato1, 5.4]).

11.3. Let $H$ be an object of $\mathscr{C}_{S}$. We denote by $P(H)$ the set of height 1 points of $H$, by

$$
P_{S}(H)=P(H) \cap H_{s}, \quad P_{\eta}(H)=P(H) \cap H_{\eta} .
$$

We have ([Kato1, 5.2], [AS4, A.6]):

(i) $H_{\eta}$ is geometrically regular over $\eta$ and for any $\mathfrak{p} \in P_{\eta}(H)$, the residue field $\kappa(\mathfrak{p})$ of $H$ at $\mathfrak{p}$ is a finite extension of the fraction field $K(S)$ of $S$.

(ii) $H_{S}$ is a reduced henselian noetherian local scheme over $s$ of dimension 1, hence $P_{S}(H)$ is a finite set.

We denote by $\widetilde{H}_{S}$ the normalization of $H_{s}$, which is a finite union of strictly henselian traits. We put

$$
\delta(H)=\operatorname{dim}_{k}\left(\mathscr{O}_{\widetilde{H}_{s}} / \mathscr{O}_{H_{s}}\right) .
$$

11.4. Let $H$ be an object of $\mathscr{C}_{S}, U$ a non-empty open sub-scheme of $H_{\eta}$ and $\mathscr{F}$ a locally constant constructible étale sheaf of $\Lambda$-modules over $U$. For a triple $(H, U, \mathscr{F})$ and a finite covering $\left(S^{\prime}, \eta^{\prime}, s^{\prime}\right)$ of $(S, \eta, s)$, we denote by $(H, U, \mathscr{F})_{S^{\prime}}$ the triple $\left(H^{\prime}, U^{\prime}, \mathscr{F}^{\prime}\right)$ where $H^{\prime}=H \otimes_{S} S^{\prime}, U^{\prime}$ is the inverse image of $U$ in $H^{\prime}$ and $\mathscr{F}^{\prime}$ is the inverse image of $\mathscr{F}$ on $U^{\prime}$. We call the triple $(H, U, \mathscr{F})$ stable if there is an étale connected Galois covering $\widetilde{U}$ of $U$ such that

(i) The pull-back of $\mathscr{F}$ to $\widetilde{U}$ is constant. 
(ii) The normalization $\widetilde{H}$ of $H$ in $\widetilde{U}$ belongs to $\mathscr{C}_{S}$ and the residue field of $\widetilde{H}$ at all points of $\widetilde{H}_{\eta}-\widetilde{U}_{\eta}$ are finite separable extensions of $\kappa(\eta)$.

Proposition 11.5 ([Kato1, 6.3]). Let $(H, U, \mathscr{F})$ be a triple as Subsection 11.4.

(i) If $(H, U, \mathscr{F})$ is stable, $(H, U, \mathscr{F})_{S^{\prime}}$ is stable for any finite covering $S^{\prime}$ of $S$.

(ii) For any triple $(H, U, \mathscr{F})$, there exist a finite covering $\left(S^{\prime}, \eta^{\prime}, s^{\prime}\right)$ of $(S, \eta, s)$ such that $(H, U, \mathscr{F})_{S^{\prime}}$ is stable.

Proposition 11.5(i) follows form ([Kato1, 5.4]) and Proposition 11.5(ii) follows form [Epp].

11.6. Let $(H, U, \mathscr{F})$ be a stable triple. For $\mathfrak{p} \in P(H)$, we denote by $\widehat{\mathcal{O}}_{H, \mathfrak{p}}$ the completion of the local ring of $H$ at $\mathfrak{p}$ and by $\kappa(\mathfrak{p})$ its residue field. For $\mathfrak{p} \in P_{S}(H)$, we denote by $\widetilde{H}_{s, \mathfrak{p}}$ the integral closure of $H_{S}$ in $\kappa(\mathfrak{p})$, which is a strictly henselian trait. Let $\operatorname{ord}_{s, \mathfrak{p}}$ be the valuation of $\kappa(\mathfrak{p})$ associated to $\widetilde{H}_{s, \mathfrak{p}}$ normalized by $\operatorname{ord}_{s, \mathfrak{p}}\left(\kappa(\mathfrak{p})^{\times}\right)=\mathbb{Z}$. We denote also by $\operatorname{ord}_{s, \mathfrak{p}}: \Omega_{\kappa(\mathfrak{p})}^{1}-\{0\} \rightarrow \mathbb{Z}$ the valuation defined by $\operatorname{ord}_{s, \mathfrak{p}}(\alpha \mathrm{d} \beta)=\operatorname{ord}_{s, \mathfrak{p}}(\alpha)$, if $\alpha, \beta \in \kappa(\mathfrak{p})^{\times}$ and $\operatorname{ord}_{s, \mathfrak{p}}(\beta)=1$. It can be canonically extended, for any integer $r>0$, to $\left(\Omega_{\kappa(\mathfrak{p})}^{1}\right)^{\otimes r}-\{0\}$. Following ([SGA7I, XVI], [Lau1] and [Kato1, 6.4]), we call the total dimension of $\mathscr{F}$ at a point $\mathfrak{p} \in P(H)$, and denote by $\operatorname{dimtot}_{\mathfrak{p}}(\mathscr{F})$ the integer defined as follows:

(i) For $\mathfrak{p} \in P_{\eta}(H)$, we put

$$
\operatorname{dimtot}_{\mathfrak{p}}(\mathscr{F})=[\kappa(\mathfrak{p}): \kappa(\eta)]\left(\operatorname{sw}_{\mathfrak{p}}(\mathscr{F})+\operatorname{rank}(\mathscr{F})\right),
$$

where $\operatorname{sw}_{\mathfrak{p}}(\mathscr{F})$ is the Swan conductor of the pull-back of $\mathscr{F}$ over $\operatorname{Spec}\left(\widehat{\mathcal{O}}_{H, \mathfrak{p}}\right) \times_{H} U$.

(ii) For $\mathfrak{p} \in P_{S}(H)$, we denote by $K_{\mathfrak{p}}$ the fraction field of $\widehat{\mathcal{O}}_{H, \mathfrak{p}}$. Since the triple $(H, U, \mathscr{F})$ is stable, there exists a finite Galois extension $L_{\mathfrak{p}}$ of $K_{\mathfrak{p}}$ of ramification index one, such that the representation $\mathscr{F}_{\mathfrak{p}}$ of $\operatorname{Gal}\left(K_{\mathfrak{p}}^{\text {sep }} / K_{\mathfrak{p}}\right)$ defined by $\mathscr{F}$ factors through the quotient $\operatorname{Gal}\left(L_{\mathfrak{p}} / K_{\mathfrak{p}}\right)$. Notice that $L_{\mathfrak{p}} / K_{\mathfrak{p}}$ factors through a field $K_{\mathfrak{p}}^{\prime}$ such that $K_{\mathfrak{p}}^{\prime} / K_{\mathfrak{p}}$ is unramified and $L_{\mathfrak{p}} / K_{\mathfrak{p}}^{\prime}$ is of type (II) (Subsection 3.3). Fixing a uniformizer $\pi$ of $R$ (also a uniformizer of $K_{\mathfrak{p}}$ ), we have $\mathrm{CC}_{\psi_{0}}\left(\mathscr{F}_{\mathfrak{p}}\right) \in\left(\Omega_{\kappa(\mathfrak{p})}^{1}\right)^{m}$ (cf. Remark 10.7). We denote by $\overline{\mathscr{F}}_{\mathfrak{p}}$ the restriction to $\operatorname{Spec}(\kappa(\mathfrak{p}))$ of the direct image of $\mathscr{F}$ under $\operatorname{Spec}\left(K_{\mathfrak{p}}\right) \rightarrow \operatorname{Spec}\left(\widehat{\mathcal{O}}_{H, \mathfrak{p}}\right)$ and by $\operatorname{dimtot}_{s, \mathfrak{p}}\left(\overline{\mathscr{F}}_{\mathfrak{p}}\right)$ the sum of $\operatorname{rank}\left(\overline{\mathscr{F}}_{\mathfrak{p}}\right)$ and the Swan conductor of $\overline{\mathscr{F}}_{\mathfrak{p}}$ over $\operatorname{Spec}(\kappa(\mathfrak{p}))$. We put

$$
\operatorname{dimtot}_{\mathfrak{p}}(\mathscr{F})=-\operatorname{ord}_{s, \mathfrak{p}}\left(\mathrm{CC}_{\psi_{0}}\left(\mathscr{F}_{\mathfrak{p}}\right)\right)+\operatorname{dimtot}_{s, \mathfrak{p}}\left(\overline{\mathscr{F}}_{\mathfrak{p}}\right) .
$$

We notice that $\operatorname{ord}_{s, \mathfrak{p}}\left(\mathrm{CC}_{\psi_{0}}(\mathscr{F})\right)$ dose not depend on the choice of $\psi_{0}(10.4 .4)$ and the choice of $\pi$.

We put

$$
\begin{aligned}
\varphi_{\eta}(H, U, \mathscr{F}) & =\sum_{\mathfrak{p} \in H_{\eta}-U} \operatorname{dimtot}_{\mathfrak{p}}(\mathscr{F}), \\
\varphi_{S}(H, U, \mathscr{F}) & =\sum_{\mathfrak{p} \in P_{S}(H)} \operatorname{dimtot}_{\mathfrak{p}}(\mathscr{F}) .
\end{aligned}
$$


LeMmA 11.7 ([Kato1, 6.5]). Let $(H, U, \mathscr{F})$ be a stable triple (Subsection 11.4), $\left(S^{\prime}, \eta^{\prime}, s^{\prime}\right)$ a finite covering of $(S, \eta, s)$. We put $\left(H^{\prime}, U^{\prime}, \mathscr{F}^{\prime}\right)=(H, U, \mathscr{F})_{S^{\prime}}$.

(i) For any $\mathfrak{p} \in P_{S}(H)$ and for the unique $\mathfrak{p}^{\prime} \in P_{S}(H)$ above $\mathfrak{p}$, we have

$$
\operatorname{dimtot}_{\mathfrak{p}}(\mathscr{F})=\operatorname{dimtot}_{\mathfrak{p}^{\prime}}\left(\mathscr{F}^{\prime}\right) .
$$

(ii) For any $\mathfrak{p} \in H_{\eta}-U$, we have

$$
\operatorname{dimtot}_{\mathfrak{p}}(\mathscr{F})=\sum_{\mathfrak{p}^{\prime}} \operatorname{dimtot}_{\mathfrak{p}^{\prime}}\left(\mathscr{F}^{\prime}\right),
$$

where $\mathfrak{p}^{\prime}$ runs over the points above $\mathfrak{p}$.

11.8. Let $(H, U, \mathscr{F})$ be a triple (Subsection 11.4). By Proposition 11.5, there exists a finite covering $\left(S^{\prime}, \eta^{\prime}, s^{\prime}\right)$ of $(S, \eta, s)$ such that $(H, U, \mathscr{F})_{S^{\prime}}$ is stable. We put

$$
\begin{aligned}
\varphi_{\eta}(H, U, \mathscr{F}) & =\varphi_{\eta^{\prime}}\left((H, U, \mathscr{F})_{S^{\prime}}\right), \\
\varphi_{s}(H, U, \mathscr{F}) & =\varphi_{S^{\prime}}\left((H, U, \mathscr{F})_{S^{\prime}}\right) .
\end{aligned}
$$

By Lemma 11.7, they don't depend on the choice of the covering $\left(S^{\prime}, \eta^{\prime}, s^{\prime}\right)$.

THEOREM 11.9 Let $(H, U, \mathscr{F})$ be a triple (Subsection 11.4), $x$ the closed point of $H$, $u: U \rightarrow H_{\eta}$ the canonical open immersion. Then we have

$$
\operatorname{dim}_{\Lambda}\left(\Psi_{x}^{0}(u ! \mathscr{F})\right)-\operatorname{dim}_{\Lambda}\left(\Psi_{x}^{1}(u ! \mathscr{F})\right)=\varphi_{s}(H, U, \mathscr{F})-\varphi_{\eta}(H, U, \mathscr{F})-2 \delta(H) \operatorname{rank}(\mathscr{F}) .
$$

PROOF. Indeed, for a stable triple $(H, U, \mathscr{F})$ and any $\mathfrak{p} \in P_{S}(H), \operatorname{dimtot}_{\mathfrak{p}}(\mathscr{F})$ is the same as Kato's definition in [Kato2, 4.4] by (10.7.4).

REMARK 11.10. The Theorem 11.9 is proved by Deligne if $\mathscr{F}$ is unramified at every point of $P_{S}(H)$ ([Lau1, 5.1.1]). In the general case, Kato proved the theorem with two different definitions of the invariant $\varphi_{s}(H, U, \mathscr{F})$ ([Kato1, 6.7], [Kato2, 4.5]). T. Saito give another proof with another definition of $\varphi_{s}(H, U, \mathscr{F})$ ([Sa1]) which corresponds to the latter definition of Kato ([Kato2, 4.5]). If $\mathscr{F}$ is of rank 1, Abbes and Saito gave a definition of $\varphi_{s}(H, U, \mathscr{F})([\mathrm{AS} 4, \mathrm{~A} .10])$ using the refined Swan conductor in their ramification theory ([AS3]), which coincides with Kato's latter definition ([Kato3, remark after 6.8]). Here, using Abbes and Saito's ramification theory, we give the definition of $\varphi_{s}(H, U, \mathscr{F})$ for any rank sheaf $\mathscr{F}$ which is equal to Kato's latter formula (Theorem 10.4).

\section{REFERENCES}

[AM] A. AbBes AND A. Mokrane, Sous-groupes canoniques et cycles évanescents $p$-adiques pour les variétés abéliennes. Inst., Hautes Études Sci. Publ. Math. 99 (2004), 117-162.

[AS1] A. AbBES AND T. SAITo, Ramification of local fields with imperfect residue fields. Amer. J. Math. 124 (2002), 879-920.

[AS2] A. AbBes AND T. SAITo, Ramification of local fields with imperfect residue fields II. Doc. Math. Extra Volume Kato (2003), 5-72. 
[AS3] A. Abbes And T. SAIto, Analyse micro-locale $\ell$-adique en caractéristique $p>0$ le cas d'un trait. Publ. Res. Inst. Math. Sci. 45 (2009), 25-74.

[AS4] A. Abbes And T. SAito, Local fourier transform and epsilon factors. Compos. Math. 146 (2010), $1507-1551$.

[AS5] A. AbBes And T. SAito, Ramification and cleanliness. Tohuku Math. J. Centennial Issue 63 (2011), No. $4,775-853$.

[BGR] S. Bosch, U. GÜntzer And R. Remmert, Non-archimedean analysis. Grundlehren der Mathematischen Wissenschaften 261, Springer-Verlag, New York, Heidelberg, Berlin, 1984.

[SGA7II] P. DELIGNE AND N. KATZ, Groupes de monodromie en géométrie algébriques.II. Séminaire de Géométrie Algébrique du Bois-Marie 1967-1969 (SGA 7 II), Lecture Notes of Mathematices 340, springer-verlag, New York, Heidelberg, Berlin, 1973.

[Epp] H. EPP, Eliminating wild ramification. Invent. Math. 19 (1973), 235-249.

[Fu] L. Fu, Etale cohomology theory. Nankai Tracts Math. World Scientific Publishing Co. Pte. Ltd., Hackensack, NJ, 2011.

[EGA IV] A. GRothendieck And J.A. DiEudonnÉ. Éléments de géometrie algébrique. IV. Étude locale des schémas et des morphisms de shémas. Inst. Hautes Études Sci. Publ. Math. 20 (1961), 24 (1965), 28 (1966), 32 (1967).

[SGA7I] A. GROTHENDIECK et al. Groupes de monodromie en géométrie algébriques.I. Séminaire de Géométrie Algébrique du Bois-Marie 1967-1969 (SGA 7 I). Dirigé par A. Grothendieck. Avec la collaboration de M. Raynaud et D. S. Rim. Lecture Notes in Mathematics 288. Sringer-Verlag, Berlin-New York, 1972.

[deJ] A. J. DE JoNG, Crystalline Dieudonné module theory via formal and rigid geometry. Inst. Hautes Études Sci. Publ. Math. 82 (1995), 5-96.

[Kato1] K. KATO, Vanishing cycles, ramification of valuations, and class field theory. Duke Math. J. 55 (1987), No. 3, 629-659.

[Kato2] K. Kato, Swan conductors with differential values. Adv. Stud. Pure Math. 12 (1987), 315-342.

[Kato3] K. KATO, Swan conductors for characters of degree one in the imperfect residue field case. Contemp. Math. 83 (1989), 101-131.

[Katz] N. KATZ, Gauss sum, Kloosterman sums, and monodromy groups. Annals of Mathematics Studies 116, Princeton University Press, Princeton, NJ, 1988.

[Lau1] G. LAumon, Semi-continuité du conducteur de Swan (d'après P. Deligne). In the Euler-Poincaré characteristic, Astérisque, 82-83 (1981), 173-219.

[Lau2] G. LAUMON, Transformation de Fourier, constantes d'équations fonctionnelles et conjecture de Weil. Inst. Hautes Études Sci. Publ. Math. 65 (1987), 131-210.

[OW] A. OBus AND S. Wewers, Cyclic extensions and the local lifting problem. 2012, arXiv:1203.5057.

[Sa1] T. SAITO, Trace formula for vanishing cycles of curves. Math. Ann. 276 (1987), 311-315.

[Sa2] T. SAITO, Wild ramification and the characteristic cycle of an $\ell$-adic sheaf. J. Inst. Math. Jussieu 8 (2009), 769-829.

[Sa3] T. SAITO, Ramification of local fields with imperfect residue fields III. Math. Ann. 352 (2012), No.3, 567-580.

[Sa4] T. SAITo, Wild Ramification and the cotangent bundle. 2013, arXiv:1301.4632v4.

[Se1] J. P. SerRe, Corps locaux. Deuxième édition, Publications de l'Université de Nancago, No.VIII. Hermann, Paris, 1968.

[Se2] J. P. SERRE, Linear representations of finite groups. Graduate Texts in Mathematics 42. Springer-Verlag, New York-Heidelberg, 1977.

[Xiao] L. XIAO, On ramification filtrations and $p$-adic differential equations, I: equal characteristic case. Algebra Number Theory 4 (2010), 969-1027. 
Institut des Hautes Études SCIENTIFIGUeS

LE BOIS-MARIE

35 RUE DE CHARTRES

91440 BURES-SUR-YVETTE

FRANCE

E-mail address: haoyu@ihes.fr

huhaoyu@mail.nankai.edu.cn 